\title{
CREDIT SUISSE
}

Facteurs de succès pour PME suisses La succession d'entreprise dans la pratique

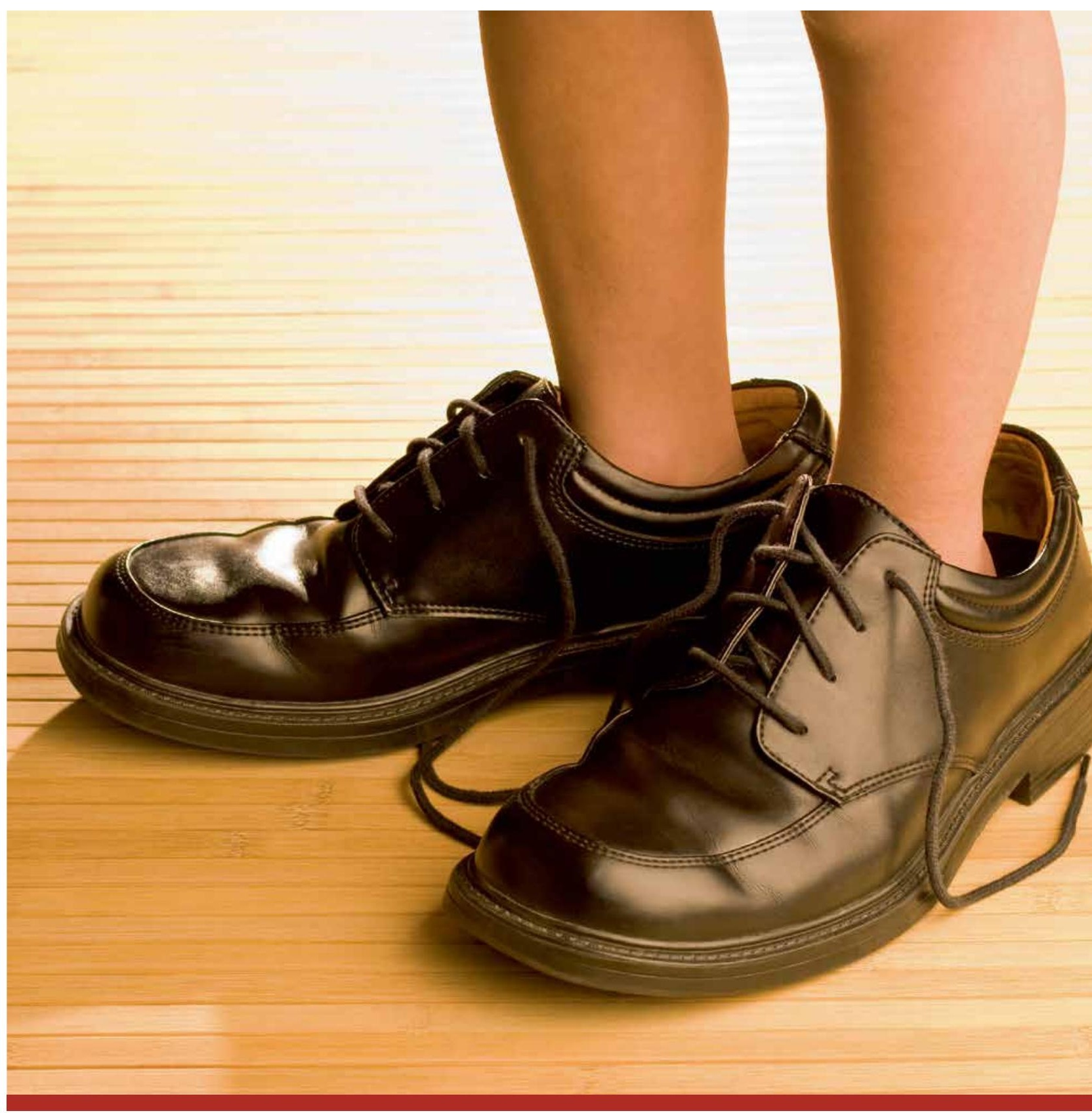




\section{Impressum}

\section{Editeur}

Giles Keating

Head of Research for Private Banking and Wealth Management

+4144332 2233

giles.keating@credit-suisse.com

Dr. Oliver Adler

Head Economic Research

+41443330961

oliver.adler@credit-suisse.com

\section{Contact}

Credit Suisse Global Research

branchen.economicresearch@credit-suisse.com

+41443347419

Center for Family Business (CFB), Université de St-Gall

frank.halter@unisg.ch

+41712247100

\section{Photo de couverture}

(C) iStockphoto.com/nfsphoto

Impression

galledia ag, Burgauerstrasse 50, 9530 Flawil

\section{Clôture de rédaction}

30 avril 2013

\section{Commandes}

Directement auprès de votre conseiller ou dans toutes les succursales du Credit Suisse

Exemplaires à l'unité (gratuit) sur www.credit-suisse.com/publications

ou fax +41443335679

Commandes internes via Myshop sous $n^{\circ}$ mat. 1511492

\section{Visitez notre site Internet}

www.credit-suisse.com/research

\section{Copyright}

Ce document peut être cité en mentionnant la source.

Copyright (C) 2013 Credit Suisse Group AG et/ou sociétés liées.

Tous droits réservés.

\section{Auteurs}

Andreas Christen, Credit Suisse Global Research

Dr. Frank A. Halter, CFB-HSG

Dr. Nadine Kammerlander, CFB-HSG

Damian Künzi, Credit Suisse Global Research

Dr. Manuela Merki, Credit Suisse Global Research

Prof. Dr. Thomas Markus Zellweger, CFB-HSG 


\section{Sommaire}

\section{Editorial}

Résumé

Informations sur l'enquête

Facteurs de succès pour PME suisses

Facteurs de succès et conditions-cadres 9

Importance et influence actuelles $\quad 10$

Evolution prévue et recommandations $\quad 15$

Complément: caractéristiques des entreprises $\quad 17$

$\begin{array}{lr}\text { Succession d'entreprise } & 19\end{array}$

Etat des lieux 19

Importance économique 19

Importance pour l'entrepreneur 22

Démographie des entrepreneurs 23

Entreprises familiales 25

Règlements de successions: souhaits et réalité $\quad 27$

Point de vue des cédants $\quad 27$

Point de vue des repreneurs $\quad 29$

Processus de transmission $\quad 32$

Sélection des candidats et rôle de l'organe de surveillance

Durée et organisation de la transmission 33

Outils pour le processus de rapprochement 34

Détermination du prix et financement 38

Evaluation de la performance de l'entreprise $\quad 40$

Informations complémentaires 


\section{Editorial}

En Suisse, une entreprise sur quatre connaîtra une transition générationnelle au cours des cinq prochaines années. La plupart de ces sociétés prévoient un transfert de gouvernance dans les deux prochaines années. Ces chiffres illustrent à quel point ce thème est actuel, sans parler de sa dimension émotionnelle. La succession d'entreprise est une affaire de cœur: bon nombre d'entrepreneuses et d'entrepreneurs suisses considèrent leur entreprise comme l'œuvre de toute une vie. Le règlement de la succession ne touche pas qu'eux et leur entreprise, mais a également des répercussions considérables sur leur famille. Notre enquête le confirme: la majorité des PME réfléchit de manière approfondie à la succession d'entreprise appropriée.

Nous avons le plaisir de vous présenter le second volet de notre série d'enquêtes «Facteurs de succès pour PME suisses», qui met cette fois l'accent sur la succession d'entreprise. Cette année encore, nombre de petites et moyennes entreprises (PME) de toute la Suisse et de toutes les branches nous ont livré leurs visions de l'avenir à long terme. Nous les en remercions toutes chaleureusement.

Dans la première partie de l'étude, les différentes entreprises évaluent les conditions-cadres propres à la Suisse. Quelles sont les attentes futures des PME pour les facteurs de succès définis? Quels sont les changements constatés par rapport à l'an passé? Vous trouverez des évaluations et des analyses sur ces sujets après un aperçu général de l'enquête.

La seconde partie de l'étude donne des éclaircissements quant au nombre d'entreprises et de postes de travail concernés, aux solutions privilégiées et aux principales raisons justifiant la transmission ou la reprise d'une entreprise. Nous faisons ensuite la jonction entre désir et réalité en nous demandant si les PME suisses sont concrètement en mesure de réaliser le règlement de succession auquel elles aspirent. A l'aide d'aspects choisis du processus de transmission, nous vous montrons comment les PME gèrent la succession d'entreprise ou respectivement comment elles s'y préparent. Les résultats de l'enquête correspondants sont inclus dans des blocs thématiques et des propositions de mesures concrètes tirées des renseignements obtenus complètent le sujet principal.

J'espère que notre étude viendra soutenir activement vos projets d'avenir et que nous aurons le plaisir de les accompagner.

Je vous souhaite une lecture passionnante et enrichissante.

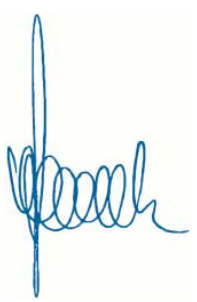

Urs Gauch

Responsable Affaires PME Suisse 


\section{Résumé}

\section{Un bon bilan des PME en Suisse malgré quelques avertissements}

(Facteurs de succès pour PME suisses, pages 9-18)

Importance capitale du thème de la succession (Etat des lieux, pages 19-25)

\section{Entreprises familiales tout particulièrement con- cernées par la succession (Entreprises familiales, pages 25-27)}

Règlement de succession: la réalité n'est pas toujours conforme aux souhaits (Règlements de successions: souhaits et réalité, pages 27-32)
Le succès et la compétitivité nécessitent sans cesse de nouveaux efforts, que ce soit pour chaque entreprise prise individuellement ou pour la place économique suisse considérée dans son ensemble. La présente étude, qui repose sur une enquête représentative effectuée auprès de plus de 2000 PME suisses, contribue au débat sur la réussite durable de la place économique suisse et examine les orientations politiques actuelles du point de vue des PME. Dans le cadre de la thématique de cette année consacrée à la succession d'entreprise, nous avons également étudié comment les PME transmettent leurs activités et leurs recettes du succès à la prochaine génération d'entrepreneurs et quels facteurs jouent un rôle important dans la pratique pour une transition aussi fluide que possible.

\section{Facteurs de succès pour PME suisses}

En 2013 aussi, les PME suisses dressent un tableau majoritairement positif des facteurs de succès. Dans l'ensemble, l'évaluation se révèle légèrement moins bonne que l'année dernière. L'enquête de cette année montre cependant que les PME suisses ressentent une dégradation de la situation pour deux facteurs déterminants, à savoir l'infrastructure et l'environnement de recherche. Ceci n'est pas vraiment surprenant. Du fait de la forte croissance démographique, I'infrastructure existante montre de plus en plus ses limites. On constate des capacités insuffisantes en matière de transport et sur le marché immobilier, la forte demande a fait grimper les prix. L'infrastructure reste toutefois le facteur ayant l'influence la plus positive sur le succès des affaires. L'environnement de recherche continue lui aussi d'avoir une influence clairement positive sur la réussite des PME. Les raisons de la dégradation observée sont moins évidentes pour ce facteur. Les PME restent néanmoins majoritairement optimistes en ce qui concerne l'infrastructure et l'environnement de recherche pour les trois à cinq prochaines années. Elles s'attendent avant tout à une dégradation des conditions-cadres réglementaires et de l'environnement économique. Compte tenu de la grande importance de ces deux facteurs, on peut parler d'avertissement, notamment dans le cas des conditions-cadres réglementaires, pour lesquelles l'Etat dispose d'un fort pouvoir d'influence.

\section{La succession d'entreprise dans la pratique}

Le thème de la succession d'entreprise est très présent pour les PME. 22\% des PME suisses prévoient de céder la propriété de leur entreprise au cours des cinq prochaines années et près de $16 \%$ dès ces deux prochaines années. Quand une entreprise rentable ne parvient pas à trouver un repreneur, cela n'affecte pas seulement l'entreprise proprement dite. C'est une perte économique en termes de postes de travail, de création de valeur et de substance fiscale. Les successions d'entreprise concernent également les partenaires commerciaux. Notre enquête révèle pourtant que seules $14 \%$ des PME préparent une stratégie pour l'éventualité où la succession d'une entreprise partenaire échouerait. Les micro-entreprises font plus souvent état de difficultés que les petites et moyennes entreprises concernant la réalisation de leur souhait de succession. Ils optent également plus souvent pour une fermeture ou une liquidation.

78\% des PME suisses sont des entreprises familiales. Elles sont tout particulièrement concernées par le thème de la succession d'entreprise, car le lien personnel et financier unissant l'entrepreneur à l'entreprise y est particulièrement fort. Leur transmission diffère donc en de multiples aspects de celle des entreprises non familiales. En moyenne, les entrepreneurs familiaux cèdent leur activité un peu plus tard que les entreprises non familiales et la période de transmission dure généralement beaucoup plus longtemps pour les entreprises familiales.

Près de la moitié des entrepreneurs familiaux prévoient de transmettre leur entreprise au sein de leur famille. En revanche, les entreprises non familiales souhaitent le plus souvent céder leur activité à des collaborateurs. Sur le plan individuel, ces souhaits ne se réalisent pas toujours. On constate toutefois que la réalité est en général relativement proche des souhaits initiaux. Près de $40 \%$ des entreprises sont transmises au sein du cercle familial (family buy-out), $40 \%$ à l'extérieur de l'entreprise (management buy-in) et $20 \%$ à des collaborateurs de l'entreprise n'appartenant pas à la famille (management buy-out). Dans la pratique, c'est surtout le ma- 
Les repreneurs familiaux bénéficient de prix particulièrement avantageux (Détermination du prix et financement, pages 38-39)

\section{L'entrepreneuriat est vu comme un privilège}

\section{Potentiel d'amélioration dans le processus de transmission}

(Processus de transmission, pages 32-39)

Les transmissions familiales ont un fonctionnement un peu différent

\section{Les successeurs externes ne recherchent pas de la substance mais un poten- tiel}

(Evaluation de la performance de l'entreprise, page 40) nagement buy-in qui intervient beaucoup plus souvent que prévu, car bon nombre d'entrepreneurs n'ayant pas encore de projets concrets concernant leur succession finissent par céder leur entreprise à un repreneur externe. Le management buy-out se réalise moins souvent que prévu. Les collaborateurs se montrent certes souvent intéressés par le principe, mais semblent généralement reculer devant la responsabilité (financière).

On constate également que les collaborateurs ne bénéficient pas de prix plus avantageux que les repreneurs externes lors du rachat d'une entreprise. Le rabais moyen sur la valeur de marché de l'entreprise s'élève à $26 \%$ pour les collaborateurs, contre 22 à $30 \%$ pour les repreneurs externes. Les repreneurs faisant partie de la famille bénéficient en revanche d'un rabais de $42 \%$ sur la valeur de marché. 20\% d'entre eux peuvent même reprendre gratuitement l'entreprise. L'analyse plus détaillée des reprises par des personnes extérieures à l'entreprise montre que seule la moitié environ des transmissions d'entreprises à un repreneur externe s'effectue sous forme de management buy-in classique. Souvent, le successeur était déjà un ami, une connaissance ou plus rarement un partenaire commercial (p. ex. client, fournisseur) de l'entrepreneur avant de reprendre l'entreprise.

La possibilité de s'épanouir personnellement est le principal motif poussant à reprendre une entreprise, loin devant l'attractivité financière. Pour un pays comme la Suisse avec des marchés du travail qui fonctionnent, une stabilité socio-politique et une société tournée vers la consommation et les loisirs, cette observation n'a rien d'atypique. Beaucoup d'entrepreneurs semblent d'ailleurs trouver concrètement cet épanouissement. Ils aiment leur métier et la grande majorité d'entre eux recommanderait l'entrepreneuriat. Ils se retirent souvent pour des raisons d'âge ou de santé. En moyenne, lors d'une transmission d'entreprise, la direction est rajeunie de 24 ans.

L'analyse du processus de transmission montre que la décision stratégique de transmission de l'entreprise n'est qu'en partie le fait des conseils d'administration. En particulier dans les entreprises familiales, le conseil d'administration a moins d'influence et il n'y a bien souvent pas de candidat alternatif au «dauphin». Mais même pour les transmissions à l'extérieur de la famille, il n'y a de candidats externes alternatifs que dans un quart des cas. De manière surprenante, les PME n'établissent aucun catalogue d'exigences concernant le successeur dans $46 \%$ des cas de transmission. Dans 60\% des cas de transmissions de PME, il n'y a même aucun plan de formation visant à faciliter l'adaptation du repreneur et à renforcer ses compétences. Le contrôle de due diligence (examen systématique des forces/faiblesses et de la valeur de l'entreprise) s'est en revanche imposé comme une pratique courante. $80 \%$ des PME en réalisent un dans le cadre de la transmission de l'entreprise. En revanche, moins de $60 \%$ des PME disposent d'un plan de communication interne ou externe concernant leur transmission, ce qui constitue un axe d'amélioration potentiel compte tenu de l'importance de la transmission de I'entreprise pour ses collaborateurs et partenaires. Seules 60\% des PME règlent les rapports devant subsister entre l'ancien entrepreneur et l'entreprise après la transmission de celle-ci (phase post-succession).

Les transmissions au sein de la famille diffèrent en de nombreux aspects des transmissions à l'extérieur de celle-ci. Les catalogues d'exigences concernant le successeur y sont plus rares, de même que les contrôles de due diligence ou les dispositions réglant la phase de postsuccession. L'ancien patron reste souvent très présent dans l'entreprise même après la transmission. II n'est pas rare qu'il y garde un bureau pendant de longues années après la reprise et exerce une influence relativement importante sur les activités de la société. Dans la très grande majorité des cas, le successeur se sent certes encouragé et soutenu par son prédécesseur. Mais la forte présence du sénior recèle un potentiel de conflit.

Des différences entre le family buy-out et les autres formes de succession apparaissent également dans la perception de l'attractivité économique de l'entreprise rachetée. Les successeurs externes estiment plus souvent que l'entreprise qu'ils reprennent est peu performante. La perception des repreneurs familiaux à cet égard est plus positive, mais est également empreinte de pression et de responsabilité sociales. Après la reprise, l'évaluation de la performance s'harmonise. Le type de règlement de la succession ne permet donc aucune déduction quant à l'attractivité de la société. 


\section{Informations sur l'enquête}

\section{Enquête PME 2013}

L'enquête PME Credit Suisse 2013 repose sur deux sondages. 2063 petites et moyennes entreprises (PME) ont pris part au sondage principal. Elles ont rempli le questionnaire de base sur les facteurs de succès en Suisse et sur des thèmes généraux relatifs à la succession d'entreprise. 523 entrepreneurs de $\mathrm{PME}^{1}$ ayant repris leur entreprise au cours des 10 dernières années ont en outre répondu à un questionnaire complémentaire avec des questions spécifiques sur la succession d'entreprise. Le nombre de réponses varie parfois. Pour chaque figure ci-après basée sur l'enquête, le nombre de participants ayant répondu à la question concernée sera donc indiqué.

L'enquête a été réalisée en janvier et février 2013 sur la base de l'anonymat par un bureau d'enquête d'opinion indépendant. Les données anonymisées ont été traitées et analysées par I'Université de St-Gall et le département Global Research du Credit Suisse.

La répartition des réponses n'est que partiellement représentative de la structure par branche et par taille issue du dernier recensement des entreprises de l'Office fédéral de la statistique (OFS) de 2008. Les entreprises industrielles et du secteur de la construction sont surreprésentées dans l'enquête par rapport à la plupart des branches de services (figure 1). De plus, l'enquête traite davantage des moyennes entreprises que des micro-entreprises (figure 2). Toutefois, ces écarts ne diminuent en rien la pertinence des résultats. Pour les calculs du chapitre "Facteurs de succès pour PME suisses», les réponses ont été pondérées en fonction de la classe de taille et de la branche en tenant compte du nombre de personnes en équivalent plein temps sur la base du recensement des entreprises de 2008. Ceci permet une plus grande représentativité des comparaisons dans le temps.

Figure 1

\section{Répartition des branches}

Part des entreprises, $n=2063$

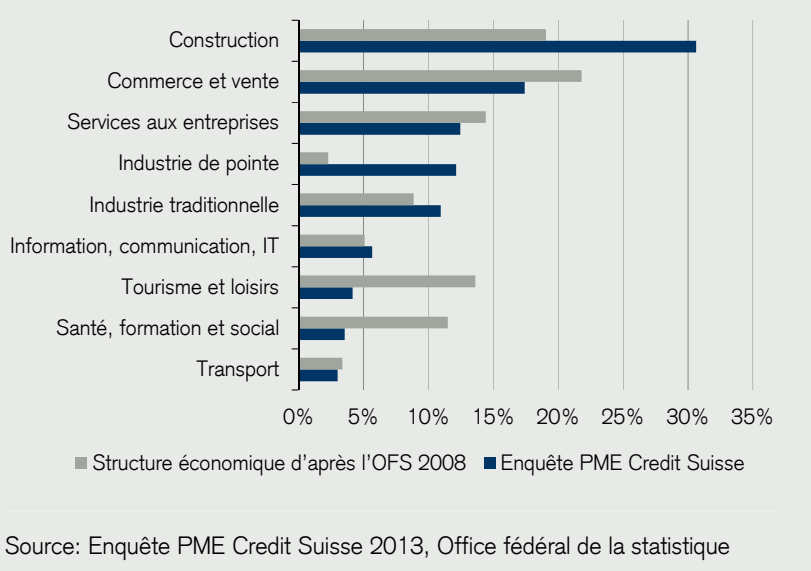

Figure 2

\section{Taille de l'entreprise}

Part des entreprises, $n=2063$

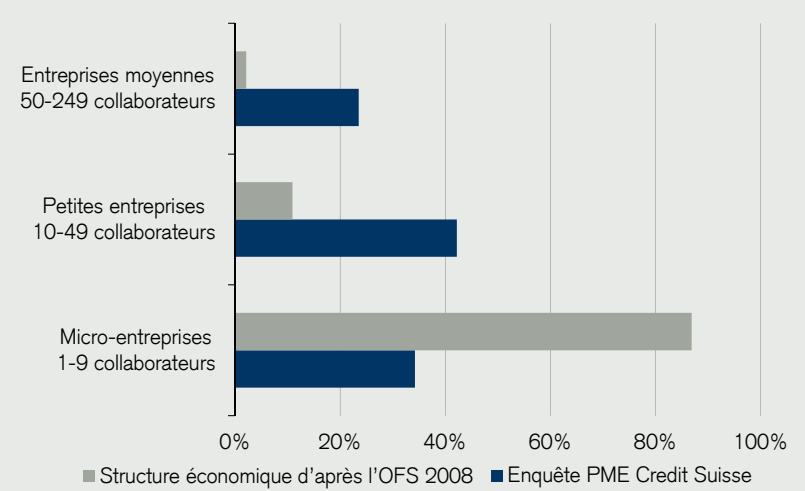

Source: Enquête PME Credit Suisse 2013, Office fédéral de la statistique 


\section{Classement par branche}

\section{Industrie de pointe}

p. ex. chimie, industrie pharmaceutique, industrie des machines, industrie automobile, industrie électronique et électrotechnique, matières plastiques, instruments de mesure et de contrôle, industrie horlogère

\section{Industrie traditionnelle}

p. ex. industrie alimentaire, meubles, papier, textile, métallurgie, produits minéraux, impression, bois, jouets, bijoux

\section{Construction}

p. ex. bâtiment, génie civil, travaux de finition et d'installation

\section{Commerce et vente}

p. ex. commerce de détail, commerce de gros, commerce auto-mobile

\section{Transports}

p. ex. transport de personnes et de marchandises, stockage, logistique, service postal et de courrier, agences de voyages

\section{Tourisme et loisirs}

p. ex. hôtellerie, restauration, organisation d'évènements culturels, services aux personnes (p. ex. coiffure, esthétique, blanchisserie)

Santé, formation et social

p. ex. médecins, thérapeutes, hôpitaux, homes, crèches, laboratoires, écoles

\section{Services aux entreprises}

p. ex. conseil, fiduciaire, activités comptables, publicité, étude de marché, gestion de bâtiments, paysagisme, courtage, recherche-développement

Information, communication et IT (TIC)

p. ex. édition, médias, communication, télécommunication, informatique

Quelle: Credit Suisse

\section{Figure 3}

\section{Part des exportations dans le chiffre d'affaires}

Part des entreprises, $n=2063$

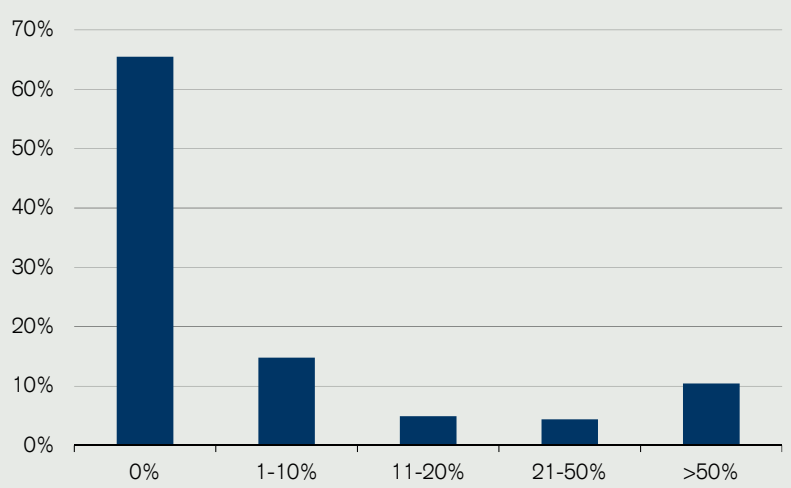

Source: Enquête PME Credit Suisse 2013
Figure 4

\section{Forme juridique}

Part des entreprises, $n=2063$

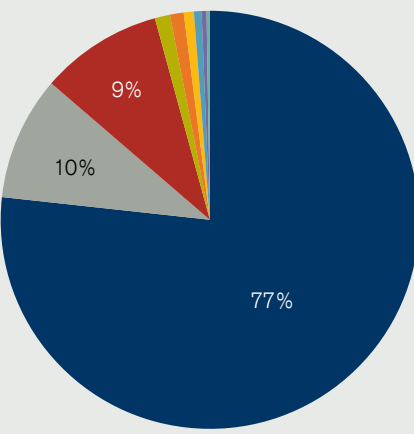

SA

SARL

Entreprise individuelle

- Société en nom collectif

- Société coopérative

Fondation

- Association

- Société en commandite

Autre

Source: Enquête PME Credit Suisse 2013

Figure 5

Autres donées structurelles

Collaborateurs (en EPT*)

Chiffre d'affaires (en mio. CHF)

Année de fondation

$\begin{array}{ccc}\text { Moyenne } & \text { Marge de variation } & \text { Médiane } \\ 39 & 0-500 & 16 \\ 14 & 0-1450 & 4 \\ 1967 & 1688-2012 & 1980\end{array}$

Source: Enquête PME Credit Suisse 2013; * EPT = Equivalent plein temps 


\section{Facteurs de succès pour PME suisses}

\section{Des facteurs internes et externes déterminent le succès de l'entreprise}

\section{Neuf facteurs de succès macroéconomiques pour les PME}

\section{Facteurs de succès et conditions-cadres}

Le succès ou l'échec d'une entreprise dépend en grande partie des décisions et compétences de l'entrepreneur. Mais les PME n'opèrent pas dans le vide: elles s'inscrivent dans un environnement économique, politique et social. De bonnes conditions-cadres facilitent le travail des entreprises. Dans un environnement adéquat, les entrepreneurs peuvent davantage se consacrer à leurs produits et clients et donc à leur véritable cœur de métier en perdant moins de temps et de ressources à cause de réglementations contraignantes, de conditions de financement difficiles, de collaborateurs non qualifiés ou d'infrastructures insuffisantes. Les possibilités pour une PME d'influer individuellement sur les facteurs externes de succès sont très limitées. Les entreprises doivent cependant se montrer réactives face à ces données et ne peuvent pas rester passives. Elles doivent exploiter à leur avantage les conditions externes positives et prendre des mesures appropriées pour réduire autant que possible les influences négatives.

Dans le cadre de la série d'études «Facteurs de succès pour PME suisses», nous interrogeons chaque année près de 2000 PME sur l'influence et l'importance des neuf principaux facteurs de succès de la place économique Suisse. Les PME évaluent la pertinence et l'influence des facteurs de succès aujourd'hui et dans le futur (figure 6). ${ }^{2}$ L'objectif est de montrer quels facteurs sont particulièrement importants pour les PME suisses et quelles conditions-cadres ont des répercussions particulièrement positives ou négatives sur l'activité d'un entrepreneur.

\section{Figure 6}

\section{Facteurs de succès des PME suisses}

\section{Infrastructure}

p. ex. transports, télécommunications et logements

\section{Ressources et environnement}

p. ex. disponibilité et prix des matières premières, approvisionnement en énergie et prix, gestion des catastrophes naturelles

\section{Conditions-cadres réglementaires}

p. ex. impôts, réglementations, collaboration avec les autorités, fédéralisme

Contexte économique

p. ex. tendances de la demande, niveau des salaires, intensité de la concurrence, stabilité des prix Interdépendance avec l'étranger

p. ex. part élevée des importations et des exportations de l'économie suisse, intégration internationale de la Suisse, taux de change, neutralité

Valeurs et société

p. ex. multiculturalisme, esprit d'entrepreneur, disposition par rapport à la réussite, tolérance au risque, culture participative

Environnement de recherche

p. ex. encouragement à l'innovation, coopération avec les hautes écoles, protection de la propriété intellectuelle, progrès technologiques

\section{Collaborateurs et qualifications}

p. ex. qualité du système éducatif, disponibilité de personnel qualifié, taux d'activité des femmes, mobilité, conscience professionnelle

\section{Conditions de financement}

p. ex. accès au marché des capitaux, niveau des intérêts, possibilités d'assurance, relation bancaire, savoir en matière d'investissement

Source: Credit Suisse

2 Les entreprises ont répondu aux quatre questions suivantes: «Comment les facteurs suivants influencent-ils aujourd'hui la réussite de votre entreprise en Suisse?» (positivement à négativement), «Quelle est actuellement l'importance des facteurs suivants pour la réussite de votre entreprise en Suisse?» (très grande à très faible), «Comment cette influence sur votre entreprise évoluera-t-elle au cours des trois à cinq prochaines années?» (positivement/négativement) et «Leur importance pour votre entreprise vat-elle augmenter ou diminuer dans les trois à cinq prochaines années?» (augmenter/diminuer). 


\section{Importance et influence actuelles}

Les facteurs ont majoritairement un effet positif sur le succès commercial
Pour beaucoup de facteurs de succès, les PME suisses ont livré en 2013 une évaluation globalement similaire à celle de l'an passé (figure 7). ${ }^{3}$ Ceci n'a rien de surprenant, car les conditionscadres ne produisent généralement leur effet sur le succès d'une entreprise qu'à moyen ou long terme et elles évoluent très rarement de manière abrupte. Comme l'année dernière, le facteur bénéficiant de l'évaluation la plus positive par les PME suisses est l'infrastructure, devant les facteurs "collaborateurs et qualification» ou «valeurs et société». Comparativement à l'an passé, les PME suisses évaluent l'environnement de recherche et l'infrastructure beaucoup moins positivement. Mais au bout du compte, la contribution de ces facteurs au succès des entreprises reste plus positive que la moyenne. Comme l'année dernière déjà, les conditionscadres réglementaires et l'interdépendance avec l'étranger sont considérées comme ayant des répercussions négatives. L'évaluation négative de l'interdépendance avec l'étranger est vraisemblablement influencée par la force persistante du franc et par les perspectives incertaines. Comme en 2012, les PME suisses ont estimé en 2013 que l'environnement économique avait une influence neutre sur leur succès commercial.

Figure 7

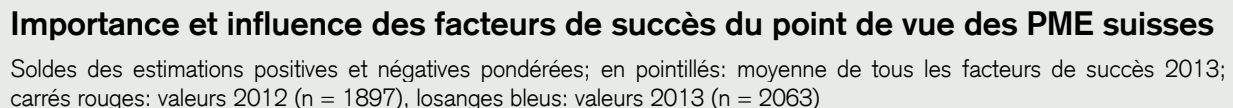
carrés rouges: valeurs $2012(n=1897)$, losanges bleus: valeurs $2013(n=2063)$

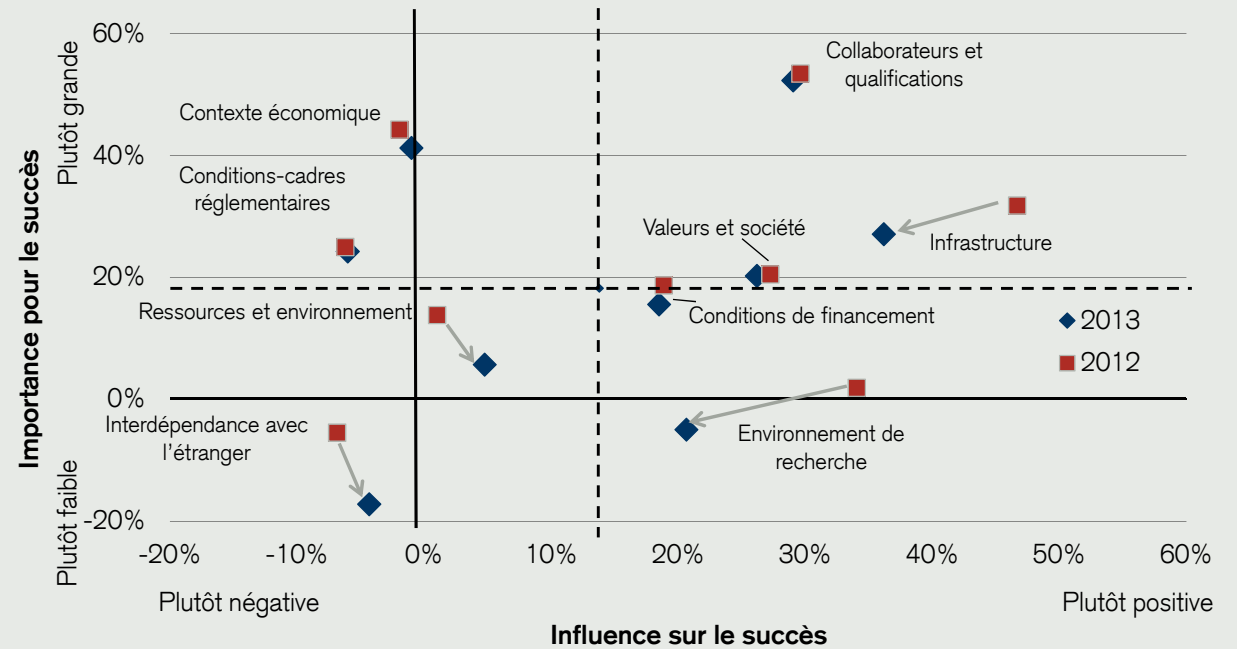

Source: Enquêtes PME Credit Suisse 2012 et 2013

En synthétisant en une seule valeur les évaluations de chacun des neuf facteurs de succès, on obtient I'«indicateur Credit Suisse de localisation pour les PME»" Cet indicateur reflète l'évaluation globale des conditions-cadres en Suisse par les PME. L'indice permet de suivre l'évolution dans le temps de l'attractivité de la Suisse en tant que lieu d'implantation pour les PME. II permet également de tirer des enseignements concernant l'attractivité relative de la place suisse pour différentes branches. Sur une échelle allant de 1 («très mauvaises conditions-cadres») à 5 ("conditions-cadres parfaites»), I'indice atteint en 2013 une valeur de 3,29 alors qu'il affichait encore 3,33 en 2012. Lorsque cette valeur est supérieure à 3, les conditions-cadres ont une influence globalement positive sur le succès commercial des PME, tandis que leur influence est négative si la valeur est inférieure à 3. Les conditions-cadres sur la place économique Suisse se sont donc dégradées de façon marginale par rapport à l'année dernière, mais au bout du comp-

3 Les conclusions s'appuient sur les soldes des réponses positives et négatives pondérées, p. ex. la part des PME considérant que le facteur de succès a une grande importance ou une influence positive, moins la part des PME considérant que le facteur de succès est plutôt peu important ou négatif. La pondération s'effectue en fonction de l'intensité indiquée pour l'évaluation. Les opinions modérées (p. ex. "plutôt faible» ou "plutôt positivement») pèsent moitié moins que les opinions tranchées ("très faible» ou «positivement»).

4 Pour cela, on prend la valeur moyenne obtenue à la question «influence sur le succès» pour chaque facteur de succès et on la pondère en fonction de l'importance accordée par les PME au facteur en question. 
De grandes différences entre les branches te elles continuent d'influer positivement sur le succès des PME. L'évaluation positive dans l'ensemble reflète la grande compétitivité de la Suisse. Dans le Rapport Global sur la Compétitivité 2012-2013 du Forum économique mondial (WEF) par exemple, la Suisse se classe une nouvelle fois première parmi les nations les plus compétitives.

L'évaluation des conditions-cadres diffère d'une branche à une autre (figure 8). L'«indicateur Credit Suisse de localisation pour les PME» atteint des valeurs supérieures à la moyenne dans les secteurs de l'information, la communication et l'informatique, des services aux entreprises, de la santé, de la formation et du social ainsi que de l'industrie de pointe. A l'autre extrémité de l'échelle, on trouve les branches du tourisme et des loisirs ainsi que du transport. Le schéma est clair: la Suisse offre de bonnes conditions-cadres avant tout aux PME des secteurs à haut niveau de connaissances.

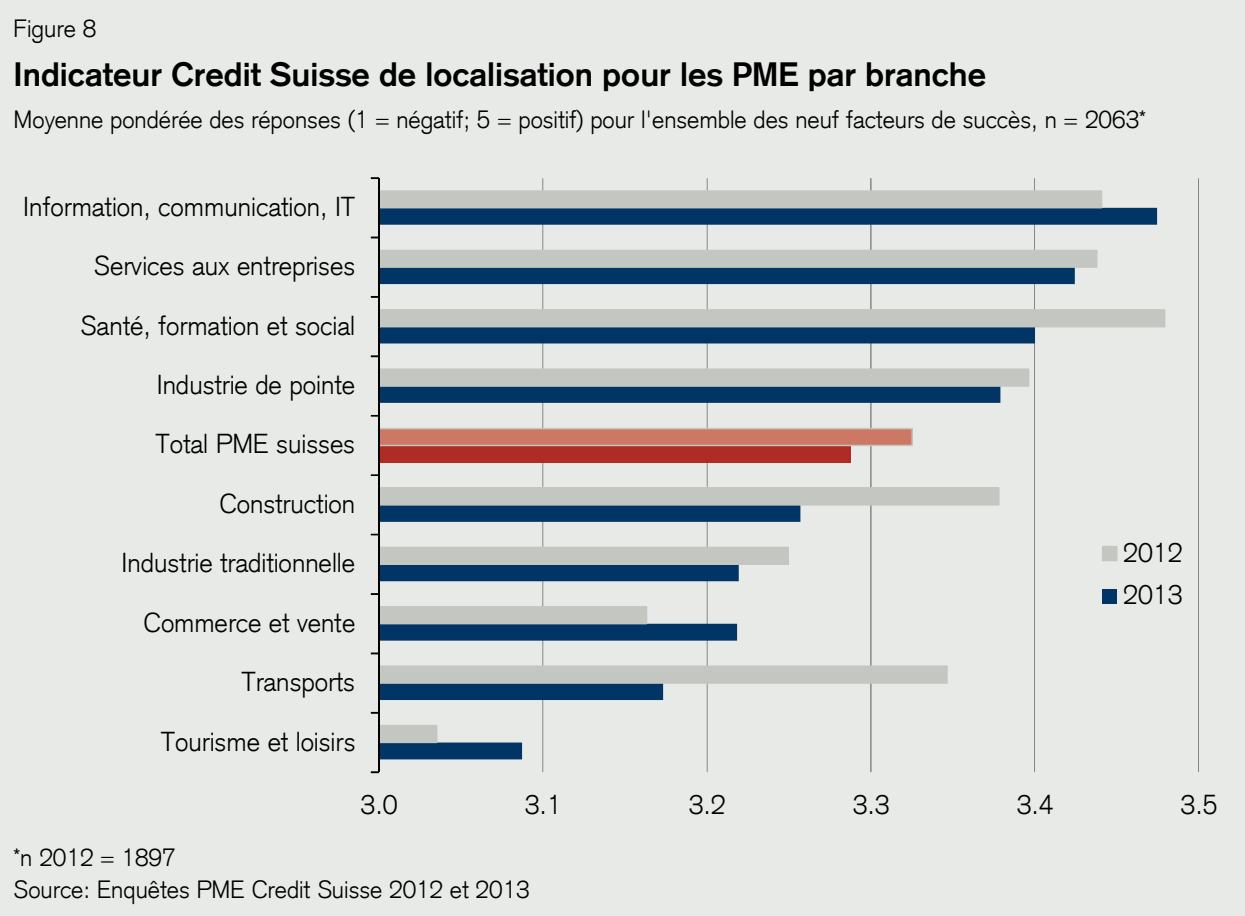

Les collaborateurs et leurs qualifications comme facteur le plus important

De multiples raisons à l'évaluation positive du facteur collaborateurs
Parmi les facteurs contribuant positivement aux bonnes conditions-cadres en Suisse selon les $P M E$, le facteur "collaborateurs et qualifications» tire à nouveau son épingle du jeu en 2013. Aucun autre facteur n'est aussi important aux yeux des PME et aucun, excepté l'infrastructure, n'influe aussi positivement sur le succès commercial. Dans toutes les branches, les entreprises attribuent une influence positive à ce facteur, qu'elles considèrent également comme le plus important de tous les facteurs. L'évaluation la plus positive du facteur avec un solde de $41 \%$ est faite par les prestataires de services aux entreprises, les PME du secteur de la santé, de la formation et du social ainsi que celles de la branche des TIC. Les PME accordant la plus grande importance à ce facteur sont celles des branches de la santé, la formation et le social, de l'industrie de pointe et des TIC.

Cette évaluation positive confirme une fois de plus l'idée courante selon laquelle la maind'œuvre suisse est qualifiée et dotée d'une grande conscience professionnelle. L'appréciation livrée par les PME illustre par ailleurs la grande confiance existant généralement entre les employeurs et les employés; un point de vue partagé par le Forum économique mondial (WEF)

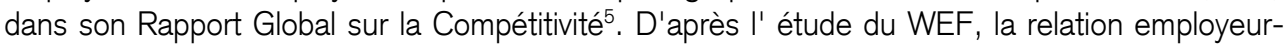
employé est plus coopérative en Suisse que dans n'importe quel autre pays du monde. Différentes études comparatives concluent en outre qu'aucun pays n'est aussi attractif que la Suisse 
pour la main-d'œuvre hautement qualifiée. L'évaluation du facteur "collaborateurs et qualification» peut également être interprétée, avec certaines réserves, comme un satisfecit donné au système éducatif suisse. Certes, dans l'enquête PME du Credit Suisse de l'année dernière, $72 \%$ des PME souhaitaient que le système éducatif soit davantage axé sur le marché du travail. ${ }^{6}$ Mais ce résultat ne doit pas être mal interprété. Les PME ne veulent pas dire par là que le système éducatif suisse est mal orienté sur le marché du travail. Des études comparatives internationales attribuent d'excellentes notes au système éducatif suisse. Dans un contexte de fort chômage des jeunes, le monde entier nous envie notre système de formation professionnelle en alternance avec son apprentissage pratique et hautement qualitatif en comparaison internationale. Ce résultat indique plutôt qu'un potentiel d'amélioration existe, certes à haut niveau. La pénurie de main-d'œuvre demeure un problème supplémentaire pour certaines branches. Ainsi, dans la précédente édition de l'étude, les PME considéraient le manque de personnel qualifié comme l'un des plus gros risques macroéconomiques.

L'infrastructure a l'influence la plus positive...
L'infrastructure a une influence légèrement plus positive encore que le facteur collaborateurs sur le succès commercial des PME suisses. Son importance est cependant moins grande sur l'ensemble des branches. Avec un solde de 49\%, les PME de la branche des TIC ont livré l'évaluation la plus positive du facteur infrastructure. Ceci n'est pas tout à fait surprenant, même si l'infrastructure TIC suisse est très bonne en comparaison internationale. Les PME du secteur du tourisme et des loisirs ont également une appréciation très positive du facteur. En comparaison internationale, l'infrastructure de transports publics est un avantage compétitif important pour le tourisme suisse. Pour ce facteur de succès, il y a davantage de différences entre les régions qu'entre les branches (figure 9). L'infrastructure est évaluée beaucoup plus positivement en Suisse centrale et à Zurich et beaucoup moins positivement dans le Tessin et dans la région lémanique que dans les autres régions de Suisse.

\section{Figure 9}

Influence de l'infrastructure: différences régionales

Soldes des réponses positives et négatives pondérées, $\mathrm{n}=2063$

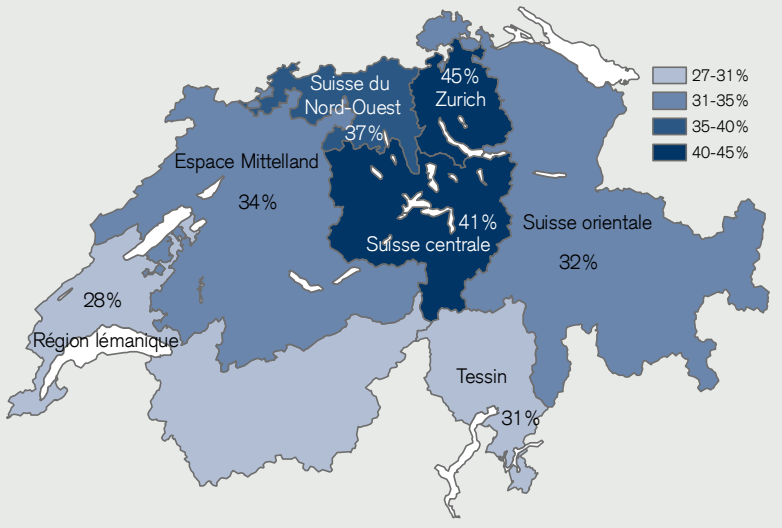

Source: Enquête PME Credit Suisse 2013

\section{Figure 10}

\section{Influence de l'infrastructure: différences entre les branches}

Soldes des réponses positives et négatives pondérées, $n=2063^{*}$

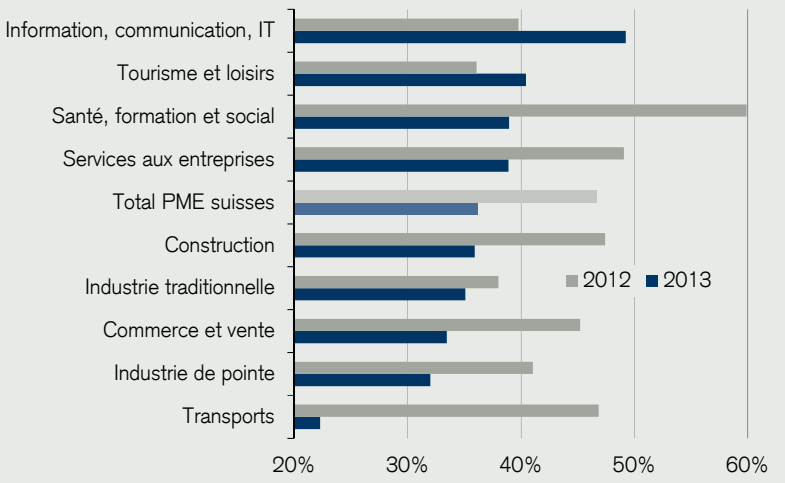

Source: Enquêtes PME Credit Suisse 2012 et 2013; "n 2012 = 1897
... mais arrive lentement à saturation de ses capacités
En 2013, les PME ont évalué l'infrastructure beaucoup plus défavorablement que l'année précédente. Cette dégradation est observable dans toutes les classes de taille et dans la plupart des branches (figure 10). Elle était prévisible, mais surprend par son ampleur. Dès 2011, I'institut lausannois IMD prévenait que l'infrastructure de transports suisse arriverait progressivement à saturation au cours des années à venir en raison de la forte croissance démographique. ${ }^{7}$ Même si pratiquement tous les classements placent encore l'infrastructure suisse parmi les meilleures du monde, celle-ci devrait se dégrader continuellement ces prochaines années à défaut d'investissements supplémentaires. Selon une étude du Fonds national, c'est surtout l'inf- 
L'interdépendance avec l'étranger a peu d'importance (directe) pour la plupart des PME... rastructure routière et ferroviaire qui manque aujourd'hui cruellement de financements en vue de son renouvellement. ${ }^{8}$ On trouve par exemple des signes mesurables de surcharge sur le réseau des routes nationales: en seulement deux ans, entre 2009 et 2011 , le nombre annuel d'heures d'embouteillage dues à la surcharge a doublé, alors que ce chiffre était resté plus ou moins stable entre 2003 et 2008. Compte tenu de l'accroissement quasi-ininterrompu du parc de véhicules automobiles, la situation s'est très vraisemblablement de nouveau aggravée en 2012. En outre, le renforcement du débat public autour du thème de l'immigration et de l'infrastructure a sans doute sensibilisé l'opinion à cette problématique.

Comme en 2012, la plupart des PME accordent au facteur de l'interdépendance avec l'étranger la plus faible importance parmi tous les facteurs de succès. En 2013, ils le considèrent même comme moins important encore que l'année dernière. Ceci reflète la bonne conjoncture intérieure, qui réduit la dépendance vis-à-vis des exportations. II faut certes admettre que la part des exportations et donc l'importance des échanges extérieurs est plus faible pour les PME que pour la moyenne de l'ensemble de l'économie. II serait toutefois faux d'en conclure que les thèmes liés au commerce extérieur tels que la force du franc suisse sont moins pertinents pour les PME. D'une part, beaucoup de PME notamment dans le domaine de l'industrie de pointe affichent une part d'exportations relativement importante (figure 11). D'autre part, bon nombre de PME sont les fournisseurs de grandes entreprises tournées vers l'export. Ainsi, dans le contexte du franc fort, certaines PME semblant de prime abord exclusivement tournées vers le marché intérieur ont perdu des commandes de grandes entreprises exposées à l'international, et ce au profit de concurrents étrangers.

\section{Figure 11}

\section{Part des exportations}

Part des entreprises avec une part des exportations supérieure/inférieure à $20 \%, n=2063$

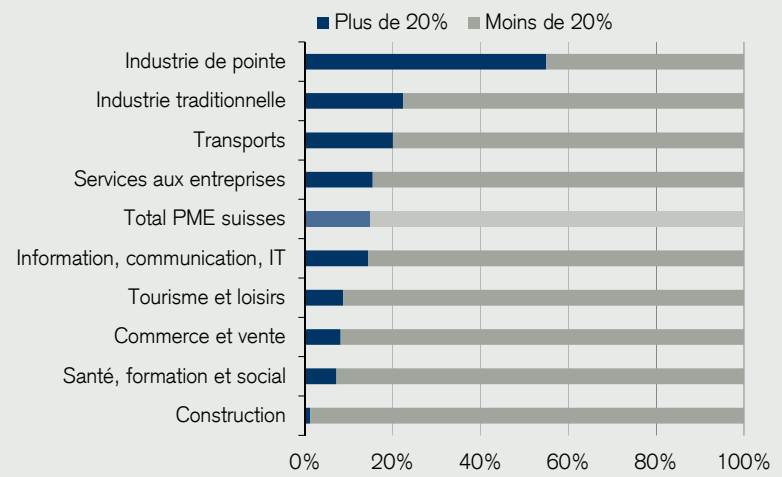

Source: Enquête PME Credit Suisse 2013

\section{Figure 12}

\section{Importance de l'environnement de recherche}

Soldes des réponses positives et négatives pondérées en $\%, n=2063$

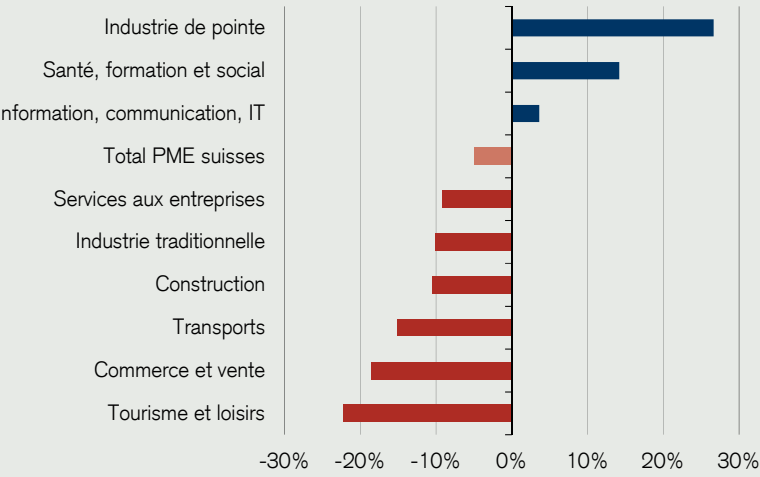

Source: Enquête PME Credit Suisse 2013

\section{... qui en font une évaluati- on relativement négative}

Mises à part les conditions-cadres réglementaires, aucun facteur de succès n'est évalué aussi négativement par les PME suisses que l'interdépendance vis-à-vis de l'étranger. Malgré l'apaisement sur le front monétaire, I'opinion des PME ne s'est améliorée que de façon marginale par rapport à 2012. Cette appréciation négative reflète la force persistante du franc ainsi que la grande incertitude relative à la conjoncture mondiale et à la crise de la dette en Europe, mais aussi la pression politique extérieure croissante exercée sur la Suisse dans différents dossiers. Le facteur est évalué de manière particulièrement négative par les PME de l'industrie traditionnelle, du commerce et de la branche du tourisme. Ces trois secteurs pâtissent de la perte de compétitivité des prix liée à la force du franc suisse. 


\section{La faible importance de l'environnement de recher- che est trompeuse}

\author{
L'environnement de re- \\ cherche envoie des impul- \\ sions positives
}

\section{L'opinion sur l'environne- ment de recherche s'est toutefois dégradée}

\section{L'esprit entrepreneurial dans la société a un effet positif}

\begin{abstract}
Des différences sectorielles et régionales dans l'évaluation de la réglementation
\end{abstract}

Comme en 2012, les PME suisses considèrent que l'environnement de recherche n'est que moyennement important. Cette appréciation ne signifie toutefois pas que les PME suisses négligent I'innovation. Mais dans la plupart des PME, l'innovation est générée en interne par des collaborateurs bien formés et non pas sur la base de collaborations avec des hautes écoles ou de programmes de soutien à l'innovation. ${ }^{9}$ Les entreprises ne bénéficient certes pas directement de l'environnement de recherche parmi les meilleurs au monde, mais elles en profitent largement de manière indirecte, notamment à travers la formation universitaire de leurs collaborateurs. Dans notre enquête, l'aspect formation est surtout pris en compte par le facteur de succès collaborateurs et qualifications. Mais dans certaines branches de PME, I'environnement de recherche a également une importance directe considérable pour le succès des affaires (figure 12). C'est particulièrement le cas pour les entreprises de l'industrie de pointe, qui accordent à ce facteur une importance relativement élevée.

Malgré l'importance (directe) plutôt moyenne de l'environnement de recherche, les PME des différentes branches attribuent à ce facteur une influence positive sur le succès commercial. Ce jugement positif est particulièrement marqué dans les branches accordant également une importance relativement élevée à l'environnement de recherche. Nous en concluons que les PME collaborant avec des hautes écoles perçoivent cette coopération comme fructueuse. Pour ce facteur de succès aussi, les PME suisses confirment donc les résultats de différentes études comparatives internationales. L'IMD World Competitiveness Yearbook 2012 place par exemple la Suisse au premier rang mondial en matière de transfert de savoir entre les universités et les entreprises.

On constate avec surprise que les PME suisses jugent dans l'ensemble beaucoup moins positivement I'influence de l'environnement de recherche en 2013 qu'en 2012 (+21\% contre $+34 \%$ ). Cette évolution de leur opinion est très difficile à expliquer. On peut imaginer qu'à travers ce jugement, les PME expriment leur scepticisme grandissant face à l'académisation de la formation professionnelle. A titre anecdotique, on peut observer qu'après un apprentissage dans une PME, certains jeunes travailleurs motivés fréquentent les hautes écoles spécialisées, où il n'est pas rare qu'ils soient débauchés par de plus grandes entreprises. Les PME perdent ainsi sans cesse leur capital humain.

Le facteur de succès «valeurs et société» exerce une influence relativement importante et fondamentalement positive sur le succès commercial. Son acception est assez large et réunit des critères tels que le multiculturalisme, l'esprit d'entrepreneur, la disposition par rapport à la réussite, la tolérance au risque et la culture participative. II constitue donc une sorte de baromètre indiquant dans quelle mesure les PME considèrent la société suisse comme «entrepreneuriale». Cette évaluation est corroborée par le fait que d'après notre enquête, près de $90 \%$ des entrepreneurs de PME suisses encourageraient leurs enfants et amis à devenirs eux-mêmes entrepreneurs (cf. chapitre «Point de vue des cédants»).

En 2013, les conditions-cadres réglementaires continuent d'être perçues par les PME suisses comme un obstacle plutôt que comme un vecteur de succès commercial (solde d'influence: $-6 \%)$. Mais cela ne signifie pas pour autant que l'environnement réglementaire en Suisse est moins propice qu'ailleurs dans le monde. La plupart des études comparatives attestent que la Suisse dispose d'un environnement réglementaire relativement favorable à l'économie. Le fait est que les interventions de l'Etat dans l'économie, aussi justifiées soient-elles d'un point de vue social, sont accueillies avec réserve par les entrepreneurs. L'évaluation du facteur réglementation diffère toutefois selon les branches. Pour les entreprises du secteur des TIC, les conditions-cadres réglementaires ont même une influence positive sur l'activité (solde d'influence: $+14 \%)$. L'évaluation la plus négative de ce facteur est à mettre au compte de la branche du tourisme et des loisirs (solde d'influence: $-25 \%$ ). Sur le plan régional, même après avoir pris en compte les différences liées aux branches, on constate que ce sont surtout les PME du Tessin et de Suisse centrale qui évaluent les conditions-cadres réglementaires plus positivement que les autres régions. Les entreprises de l'Espace Mittelland et de la région lémanique font en revanche une évaluation légèrement moins bonne de ce facteur. 


\section{Evolution prévue et recommandations}

Les PME prévoient tendanciellement une dégradation des conditions-cadres
Concernant l'avenir, les PME suisses tablent sur une dégradation des conditions-cadres pour l'ensemble des facteurs de succès ces 3 à 5 prochaines années. Comme pour l'évaluation des conditions-cadres actuelles avec l'«indicateur Credit Suisse de localisation pour les PME», nous synthétisons les attentes des PME pour le futur dans un indicateur unique. ${ }^{10}$ Sur une échelle allant de $-100 \%$ (toutes les PME prévoient une influence plus négative pour tous les facteurs de succès) à $+100 \%$ (toutes les PME prévoient une influence plus positive pour tous les facteurs de succès), cet indicateur atteint en 2013 une valeur de $-9 \%$, soit une progression marginale par rapport aux $-11 \%$ enregistrés I'année dernière. En 2013, les entrepreneurs suisses sont donc pessimistes quant à l'évolution future de la place économique suisse pour les PME, même s'ils le sont un peu moins qu'en 2012. Comme l'année dernière, les PME estiment au fond que tous les facteurs de succès auront davantage d'influence qu'aujourd'hui sur leur succès commercial (figure 13). Les PME tablent sur une amélioration des conditions-cadres concernant l'environnement de recherche, l'infrastructure, les valeurs et la société ainsi que les collaborateurs et les qualifications. Elles prévoient avant tout une dégradation en ce qui concerne l'environnement économique et les conditions-cadres réglementaires (figure 14). L'amélioration par rapport à 2012 s'explique notamment par des perspectives légèrement moins négatives concernant l'environnement économique futur et l'interdépendance avec l'étranger.
Figure 13

\section{Changement d'importance attendu 2013-2018}

Part des réponses (positives/négatives) en pourcentage, classement d'après le solde, $n=2063$

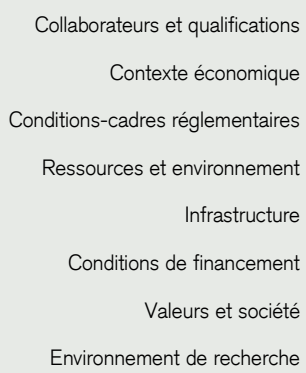

Source: Enquête PME Credit Suisse 2013
Figure 14

\section{Changement d'influence attendu 2013-2018}

Part des réponses (positives/négatives) en pourcentage, classement d'après le solde, $n=2063$

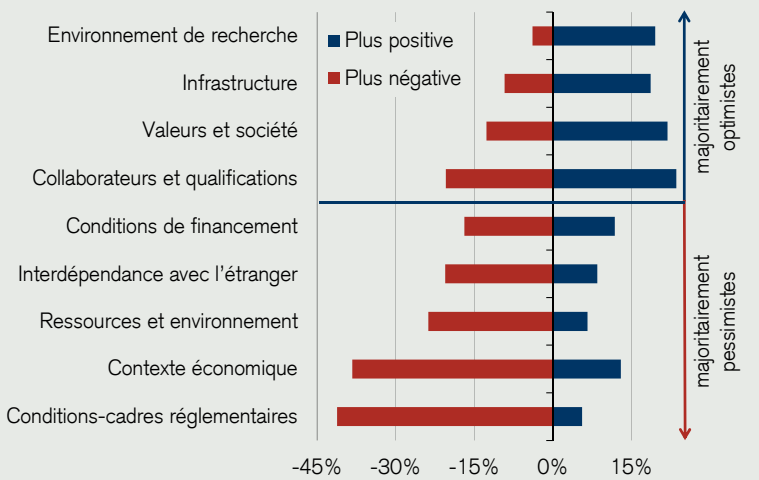

Source: Enquête PME Credit Suisse 2013
Seule la branche des TIC ne prévoit pas de dégradation des conditions-cadres
Pour ce qui est des perspectives d'avenir, on constate certaines différences entre les branches. Les PME de la branche des TIC sont les plus «optimistes» avec un indicateur de 0\%. Les PME de cette branche considèrent donc dans l'ensemble que les conditions-cadres en Suisse ne connaitront aucune dégradation ni amélioration à moyen terme. Derrière les entreprises de la branche des TIC, on trouve les PME du secteur de la santé, de la formation et du social ainsi que celles de l'industrie de pointe avec un indicateur de $-4 \%$. Les entreprises les plus pessimistes sont celles des secteurs de l'industrie traditionnelle $(-14 \%)$ et du commerce $(-13 \%)$. Ces différences ne doivent rien au hasard: le thème de la santé est considéré comme la prochaine grande tendance. Les innovations techniques ouvrent sans cesse de nouvelles possibilités dans ce domaine. Parallèlement, le facteur santé prend de plus en plus d'importance sur le plan social et individuel. C'est pourquoi les PME du domaine de la santé sont également plus optimistes que la moyenne en ce qui concerne les facteurs environnement de recherche et valeurs et société. 
Les collaborateurs et qualifications restent un des piliers du succès

\section{Optimisme malgré une surcharge croissante de l'infrastructure}

\section{Les PME craignent une dé- gradation de l'environne- ment réglementaire}

\author{
Le pessimisme reste de \\ mise concernant l'environ- \\ nement économique et \\ l'interdépendance avec \\ l'étranger
}

Le capital humain est le facteur de succès pour lequel le plus grand nombre de PME prévoient une importance croissante. Les entrepreneurs s'attendent par ailleurs à ce que son influence future sur leur succès commercial soit plus positive qu'aujourd'hui (solde: $+3 \%$ ). Ce résultat envoie un signal sans équivoque aux entreprises et aux décideurs politiques: le facteur capital humain est déterminant pour l'avenir de la place suisse comme lieu d'implantation des PME. Les entreprises sont d'une part tenues d'exploiter au mieux les excellentes conditions-cadres actuelles. Pour attirer une main-d'œuvre qualifiée, elles doivent offrir des conditions de travail attractives sur le plan financier mais aussi et surtout sur le plan non financier. D'autre part, dans leur propre intérêt sur le long terme, les entreprises ont le devoir de contribuer elles-mêmes au facteur de succès collaborateurs en proposant des stages et des places d'apprentissage et en aidant autant que possible leur personnel à se perfectionner. Les décideurs politiques ne doivent pas non plus s'endormir sur leurs lauriers. Le système éducatif suisse est excellent mais il peut encore être amélioré, par exemple en termes d'efficacité. Du point de vue des PME, la formation doit être davantage axée sur le marché du travail. Ce sont précisément les PME qui comptent sur le système de formation professionnelle en alternance et elles n'auraient aucun intérêt à une académisation trop importante de la formation professionnelle. L'immigration de maind'œuvre étrangère ne suscite que partiellement l'adhésion des entrepreneurs de PME. ${ }^{11}$ "Grow your own»: tel est le souhait adressé par les PME au monde politique.

Même si les PME suisses font en 2013 une évaluation un peu moins positive de l'infrastructure qu'en 2012, elles sont modérément optimistes pour le futur dans l'ensemble des branches (solde: $+9 \%)$. Les PME sont manifestement une majorité à penser que les problèmes qui se sont profilés ces dernières années peuvent être surmontés. Mais pour préserver l'excellente qualité de l'infrastructure suisse y compris dans le futur, des efforts sont indispensables. Selon les prévisions, la population va continuer de croître fortement ces prochaines années, ce qui accentuera la surcharge de l'infrastructure des transports et aggravera encore les problématiques du mitage et de la pression foncière dans les centres. Cela nécessite d'une part d'engager davantage de moyens pour l'infrastructure des transports. II existe d'autre part toute une série de mesures qui devraient permettre de désamorcer ces problématiques en engageant relativement peu de ressources. L'endiguement du mitage est un point de départ essentiel. En approuvant la révision de la Loi sur l'aménagement du territoire en mars 2013, le peuple a envoyé un signal en ce sens. Mais cela ne suffira sans doute pas. L'une des mesures les plus efficaces réside dans la densification des constructions: tout au moins dans un contexte marqué par la saturation progressive de l'infrastructure des transports et par la pression foncière, il devrait être possible à l'avenir de construire davantage en hauteur dans les villes suisses, sous réserve de tenir compte du paysage urbain et de préserver la qualité de vie. ${ }^{12}$

Début 2012, c'était pour l'environnement économique que les PME réservaient leurs prévisions les plus pessimistes. En 2013, ce sont les conditions-cadres réglementaires qui sont la nouvelle lanterne rouge: ceci indique que les PME sont extrêmement sceptiques face à la judiciarisation en cours de l'économie et de la société. On peut en outre supposer qu'elles craignent une dégradation de la situation fiscale dans le futur. Le message adressé à l'Etat par les PME est donc le suivant: il faut user avec circonspection des impôts et réglementations supplémentaires.

Bien que le pessimisme des PME concernant l'environnement économique ait légèrement reculé par rapport à l'année dernière, celles-ci tablent toujours sur une dégradation de la situation. Les prévisions toujours aussi pessimistes concernant l'interdépendance avec l'étranger malgré une éclaircie indiquent que les PME ne comptent pas sur une résolution rapide de la crise de l'euro. Ce pessimisme se retrouve dans toutes les branches, mais c'est dans la branche de la construction et dans l'industrie traditionnelle qu'il est le plus manifeste. 


\section{Complément: caractéristiques des entreprises}

Les caractéristiques des entreprises influent sur l'évaluation des conditionscadres

Les grandes PME attribuent une plus grande importance aux facteurs de succès

\section{Les petites PME font une évaluation moins positive des facteurs de succès}

Les facteurs de succès peuvent avoir une influence différente suivant l'entreprise. Nous avons montré que selon la branche, les PME évaluent de manière systématiquement différente les facteurs de succès. Mais outre la branche d'appartenance, d'autres caractéristiques des entreprises ont une influence sur la manière dont les conditions-cadres se répercutent sur le succès commercial. Ci-dessous, nous abordons deux de ces caractéristiques: la taille de l'entreprise et le fait que l'entreprise soit ou non dirigée par son fondateur.

Notre enquête montre clairement que la taille des entreprises influence significativement leur évaluation des facteurs de succès. Plus l'entreprise est grande, plus l'importance de tous les facteurs de succès augmente (figure 15). Dans les petites sociétés, le succès dépend souvent plus des décisions individuelles de l'entrepreneur que dans les grandes. Plus une société est grande, plus elle s'inscrit dans différents systèmes extérieurs à l'entreprise et donc moins elle est flexible. Ou dit de manière imagée: plus le navire est grand, plus les manœuvres sont lourdes pour changer de cap en cas d'évolution des conditions climatiques.

Les grandes PME attribuent non seulement un plus grand poids mais aussi une influence plus positive aux facteurs de succès que les entreprises de plus petite taille (figure 16). Ceci vaut pour les facteurs infrastructure, valeurs et société, environnement de recherche, collaborateurs et qualifications, et conditions de financement. Néanmoins, les micro-entreprises évaluent en fin de compte elles aussi positivement tous les facteurs de succès cités. II est assez surprenant d'observer que, tout au moins parmi les PME, la taille de l'entreprise n'a pas d'influence significative sur la manière dont celle-ci évalue le contexte réglementaire. Ceci contredit quelque peu l'idée communément admise selon laquelle les réglementations accablent davantage les petites entreprises que les grandes du fait de la charge administrative. II convient cependant de rappeler que le facteur des conditions-cadres réglementaires tel que défini ici inclut non seulement les réglementations au sens strict du terme mais aussi le système fiscal, la collaboration avec les autorités et le fédéralisme.
Figure 15

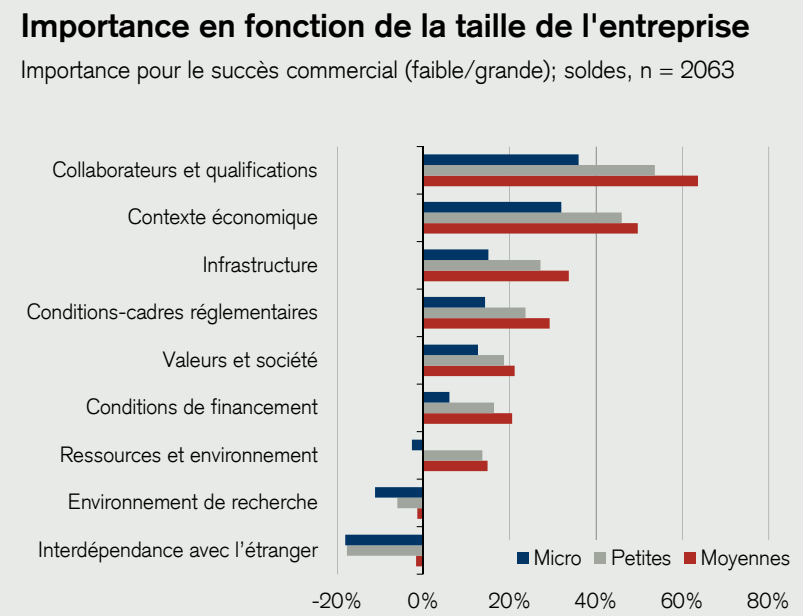

Source: Enquête PME Credit Suisse 2013
Figure 16

\section{Influence en fonction de la taille de l'entreprise}

Influence sur le succès commercial (négative/positive); soldes, $n=2063$

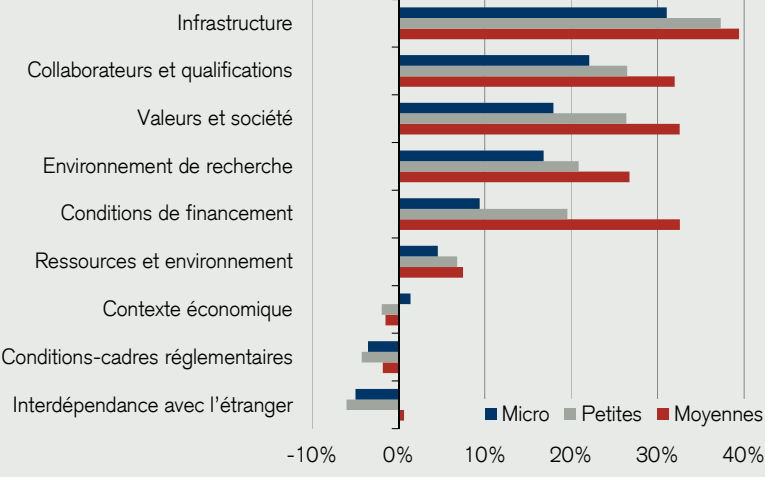

Source: Enquête PME Credit Suisse 2013
Les entreprises de fondateurs tendanciellement plus optimistes...
Le thème principal de cette étude est la succession d'entreprise. Dans ce contexte, il est intéressant de se demander si les entrepreneurs-fondateurs font ou non la même évaluation des facteurs de succès suisses que les entrepreneurs non fondateurs. II existe en effet certaines différences, même si elles ne sont statistiquement significatives que pour quelques facteurs de succès. Les fondateurs d'entreprises jugent moins négative l'influence de l'interdépendance avec l'étranger et moins positive celle des conditions de financement que les entrepreneurs non fondateurs, et ce, même si l'on tient compte des différences liées à la branche, à l'âge et à la taille de l'entreprise. Les fondateurs d'entreprises considèrent l'environnement économique et le facteur collaborateurs comme légèrement moins importants, I'environnement de recherche 
... car plus proches des tendances technologiques comme plus important que ne les considèrent les entreprises qui ne sont pas dirigées par leur fondateur. En ce qui concerne l'influence future, les fondateurs sont un peu plus optimistes ou respectivement moins pessimistes que les non fondateurs pour tous les facteurs de succès, même si ce schéma n'est statistiquement significatif que pour l'environnement économique.

Si les entrepreneurs-fondateurs sont plus optimistes quant à l'environnement économique futur, c'est vraisemblablement parce qu'ils ont tendance à jouer un rôle d'innovation ou d'avant-garde technologique sur leur marché partiel, ce qui les protège un peu mieux de la concurrence. L'importance relativement élevée accordée par ce type d'entreprises à l'environnement de recherche corrobore cet argument. 


\section{Succession d'entreprise}

\section{Etat des lieux}

\section{Importance économique}

\author{
Un thème d'importance \\ macroéconomique et indi- \\ viduelle
}

\section{L'échec d'une succession détruit de la valeur}

\section{L'enquête PME du Credit Suisse 2013 permet de tirer des conclusions actuelles}

$22 \%$ des PME prévoient une succession d'ici à 2018
La pérennisation de l'activité et la recherche d'un règlement de succession approprié constituent pour chaque entreprise un défi très particulier. La réussite des PME suisses repose sur des conditions-cadres favorables (cf. chapitre "Facteurs de succès»). Le micro-environnement et les facteurs internes à l'entreprise sont tout aussi importants. Mais en l'absence de successeur, même une entreprisse florissante bénéficiant d'un environnement favorable peut disparaître. La succession d'entreprise n'est d'ailleurs pas seulement un événement décisif pour les entreprises concernées, mais revêt aussi une grande importance sur le plan macroéconomique.

Lorsque des entreprises rentables échouent à se transmettre, cela détruit de la valeur macroéconomique. Des emplois sont perdus. Des partenaires commerciaux, clients et fournisseurs doivent se réorienter. Les raisons conduisant à une fermeture ou une liquidation ne sont certes pas toujours très claires et le lien est vite fait entre manque de potentiel commercial et absence de successeur. Mais le risque de perte de valeur macroéconomique suite à l'échec de successions d'entreprise est bien réel. Dans la littérature spécialisée, on estime qu'il y a jusqu'à $30 \%$ d'entreprises qui ne sont pas transmises et disparaissent par conséquent.

Les informations recueillies dans le cadre de l'enquête PME de cette année nous permettent de quantifier l'importance macroéconomique de la succession d'entreprise et de tirer de nouveaux enseignements concernant le processus de succession d'entreprise. Le Center for Family Business de I'Université de St-Gall a déjà étudié ce sujet en collaboration avec le Credit Suisse en $2009 .{ }^{13}$ La présente étude s'appuie sur les résultats obtenus alors et les actualise, met en évidence les évolutions et approfondit les connaissances relatives au contexte et aux corrélations.

D'après notre enquête de cette année, $22 \%$ des PME suisses prévoient de céder la propriété de leur entreprise au cours des cinq prochaines années (figure 17). Près de $16 \%$ envisagent de le faire dès ces deux prochaines années. Une proportion un peu plus élevée de PME souhaite transmettre la direction de l'entreprise: $25 \%$ d'ici cinq ans et 17\% d'ici deux ans. Beaucoup plus d'entreprises, à savoir $73 \%$ d'entre elles, indiquent avoir déjà pensé au règlement de leur propre succession, ne serait-ce que dans les grandes lignes (figure 19).

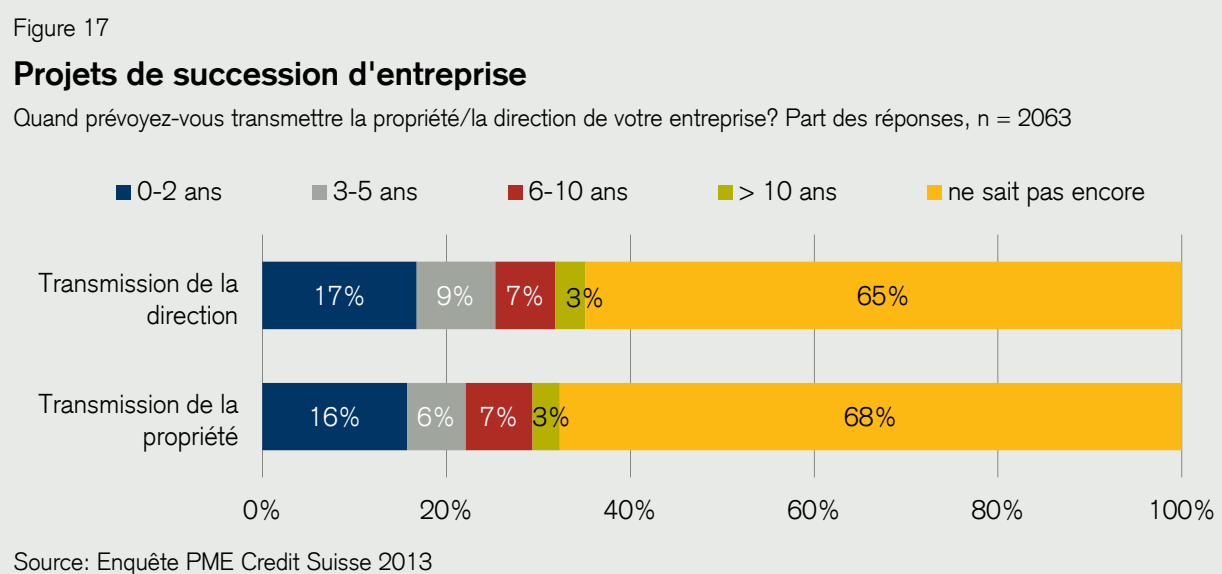


Près d'un demi-million d'emplois concernés

Les baby-boomers et la peur de l'impôt sur les successions influent sur le taux de succession

Les plus grandes entreprises ont plus souvent des projets de transmission...
En chiffres absolus extrapolés pour l'ensemble de l'économie nationale, cela signifie la chose suivante: au cours des cinq prochaines années, 466000 postes de travail dans près de 71000 entreprises devraient être concernés par un transfert de propriété et près de 534000 emplois dans 81000 entreprises par un transfert de direction (figure 18).

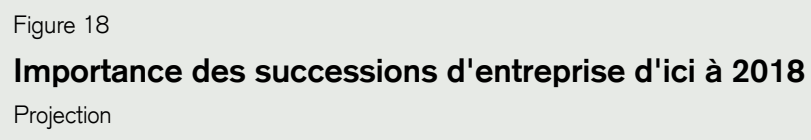

\begin{tabular}{|c|c|c|c|c|c|c|c|c|}
\hline \multirow[b]{2}{*}{$\begin{array}{l}\text { Taille de } \\
\text { l'entreprise }\end{array}$} & \multicolumn{2}{|c|}{ Economie nationale } & \multicolumn{3}{|c|}{ Transmission de la propriété } & \multicolumn{3}{|c|}{ Transmission de la direction } \\
\hline & PME & $\begin{array}{l}\text { Employés } \\
\text { (EPT) }\end{array}$ & $\begin{array}{l}\text { Succession } \\
<5 \text { ans }\end{array}$ & PME & $\begin{array}{l}\text { Employés } \\
\text { (EPT) }\end{array}$ & $\begin{array}{l}\text { Succession } \\
<5 \text { ans }\end{array}$ & PME & $\begin{array}{l}\text { Employés } \\
\text { (EPT) }\end{array}$ \\
\hline 0-9 & $277^{\prime} 100$ & $714^{\prime} 841$ & $18 \%$ & $51^{\prime} 000$ & $131^{\prime} 700$ & $20 \%$ & $55^{\prime} 100$ & $142^{\prime} 000$ \\
\hline $10-49$ & $35^{\prime} 700$ & $699^{\prime} 054$ & $25 \%$ & $9^{\prime} 000$ & $175^{\prime} 700$ & $28 \%$ & $10^{\prime} 000$ & $194^{\prime} 800$ \\
\hline $50-249$ & $7^{\prime} 400$ & $691^{\prime} 081$ & $23 \%$ & $1^{\prime} 700$ & $160 ' 300$ & $31 \%$ & $2^{\prime} 300$ & $213^{\prime} 700$ \\
\hline Total & $320 ' 200$ & 2' 104'976 & $22 \%$ & $70^{\prime} 900$ & $466^{\prime} 000$ & $25 \%$ & $81^{\prime} 200$ & $533^{\prime} 700$ \\
\hline
\end{tabular}

Source: Office fédéral de la statistique, Enquête PME Credit Suisse 2013

Comparativement à la dernière étude datant de 2009, la part des entreprises ayant des projets de succession plus ou moins concrets pour ces cinq prochaines années a baissé de 4 points. A l'époque, 26\% des PME envisageaient de céder la propriété de l'entreprise dans un délai de cing ans. Avec $22 \%$, le taux de succession actuel ${ }^{14}$ reste cependant supérieur au taux de $18,5 \%$ établi lors d'une étude comparable datant de $2005 .{ }^{15}$ Les fluctuations en matière de projets de transmission peuvent s'expliquer principalement par la situation démographique et réglementaire. Le fait que les classes d'âge les plus anciennes de la génération du baby-boom atteignent actuellement l'âge de la retraite peut certes expliquer la hausse du taux de transmission par rapport à 2005, mais le recul observé par rapport à 2009 a de quoi surprendre. Des modifications réglementaires fournissent une explication plausible à cette évolution: I'initiative pour un impôt sur les successions, et en particulier l'annonce d'une introduction rétroactive au début de l'année 2012 en cas d'acceptation, a vraisemblablement poussé certains entrepreneurs à céder plus tôt que prévu la propriété de leur entreprise et à privilégier la transmission d'entreprise. A l'inverse, on sait que les incertitudes liées à l'arrêt du Tribunal fédéral de 2004 (ATF 2A.331/2003) ${ }^{16}$ ont entraîné un report des règlements de succession et ont fait baisser le taux de succession en 2005. On ne peut pas non plus complètement exclure que certains effets aient été induits par la formulation de l'enquête.

Les grandes entreprises disposent beaucoup plus souvent de projets de transmission concrets que les petites (figure 19). Les micro-entreprises sont notamment plus nombreuses que la moyenne à ne pas savoir quand elles souhaitent transmettre l'entreprise ou à ne pas avoir encore réfléchi au règlement de leur succession. Elles ont davantage de difficultés à transmettre leur activité que les petites et moyennes entreprises. La part d'entreprises ne pouvant pas réaliser la solution souhaitée s'élève à $5,7 \%$ pour les micro-entreprises, soit plus que pour les petites et moyennes entreprises (respectivement $4,4 \%$ et 2,6\%). Les micro-entreprises optent par ailleurs plus souvent (8\%) pour une liquidation que les petites et moyennes entreprises (respectivement $2 \%$ et $0 \%$ ). Ceci est vraisemblablement dû au fait que leur destin soit directement lié à la personne de l'entrepreneur et qu'elles opèrent souvent à la limite du seuil de rentabilité, ce qui rend difficile la transmission de l'entreprise.

16 A l'époque, la réinterprétation de la liquidation partielle indirecte par le Tribunal fédéral aurait signifié dans le cadre d'une cession/reprise la taxation de la part financée non pas sur fonds propres mais par des fonds de tiers (y compris les cofinancements par le cédant via un prêt ou un avancement d'hoirie), et ce, directement lors de l'aliénation des participations. Pour les entreprises familiales, cela serait revenu dans les cas extrêmes à imposer l'intégralité de la valeur ajoutée créée par une génération. 
Figure 19

\section{Avez-vous déjà réfléchi au règlement de votre prop- re succession?}

Part des entreprises, $n=2063$

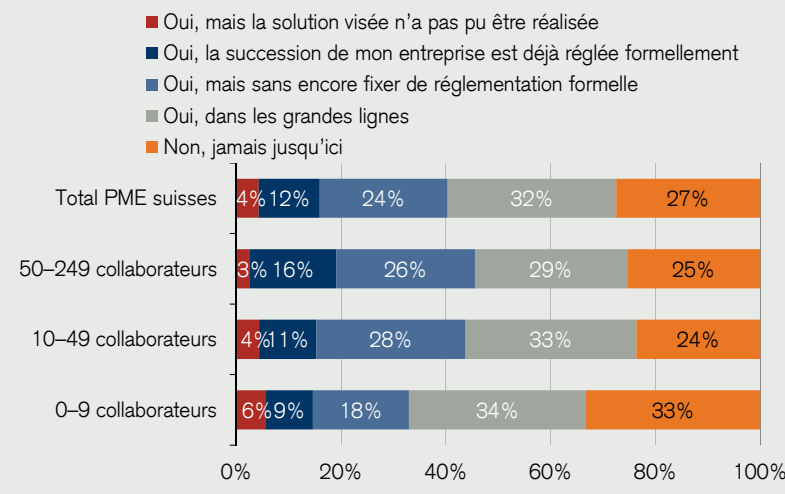

Source: Enquête PME Credit Suisse 2013
Figure 20

\section{Taux de succession par branche}

Part des entreprises ayant des projets de transmission pour les 5 prochaines années, $n=2063$

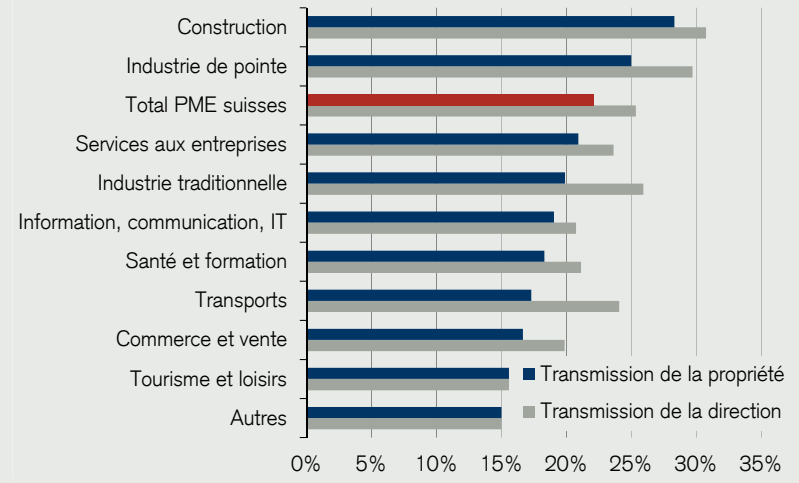

Source: Enquête PME Credit Suisse 2013
... et séparent plus souvent la transmission de la direction de celle de la propriété

Les entreprises industrielles et de construction ont plus de projets de succession

Le règlement de succession préoccupe aussi les partenaires commerciaux
Plus l'entreprise est grande, plus l'écart est important entre la transmission de la direction et celle de la propriété (figure 18). Certes, la part d'entreprises envisageant une transmission de direction est dans l'ensemble légèrement plus élevée que celle des entreprises avec des projets de transmission de propriété. Mais cet écart grandit à mesure que la taille des entreprises considérées augmente. La part des entreprises ayant des projets de transmission de direction est supérieure de $7,7 \%$ à celle des entreprises ayant des projets de transmission de propriété parmi les entreprises de 50 à 250 collaborateurs, tandis que l'écart est de 1,5\% pour les microentreprises (19,9\% contre 18,4\%). Pour les petites entreprises, la différence s'établit à 2,8\% soit un niveau intermédiaire. A mesure que la taille de l'entreprise augmente, la direction semble prendre plus d'importance que la propriété. Ceci est d'une part lié au capital plus élevé des grandes entreprises, qui constitue un obstacle relativement important lorsqu'il s'agit de financer l'acquisition de la propriété. D'autre part, les propriétaires de grandes entreprises peuvent souvent considérer cette propriété comme un placement et donc préférer transmettre uniquement la direction de l'entreprise. A une époque où les taux d'intérêt et les rendements du marché des capitaux sont bas, cette solution est particulièrement attrayante.

Le taux de succession varie beaucoup selon la branche (figure 20). Les entreprises de construction sont particulièrement nombreuses à envisager une transmission au cours des prochaines années. Leurs taux de succession en matière de transmission de propriété et de direction sont supérieurs de près d'un cinquième à ceux de l'ensemble de l'économie. Les entreprises du tourisme et des loisirs sont en revanche près de deux fois moins nombreuses que la moyenne des PME suisses à avoir des projets de succession. Ces différences significatives s'expliquent en partie par la démographie des entrepreneurs (cf. chapitre «Démographie des entrepreneurs»). Par ailleurs, le cycle économique des différentes branches joue vraisemblablement lui aussi un rôle. La branche de la construction traverse une sorte de super cycle. Elle n'en finit pas de croitre, et avec elle les craintes d'une surchauffe. Bon nombre d'entrepreneurs doivent donc songer à réaliser la valeur de leur entreprise. Dans le domaine du tourisme en revanche, une cession apparaît moins lucrative compte tenu du contexte défavorable.

Dans une optique macroéconomique, au-delà du taux de succession, il convient de ne pas perdre de vue la chose suivante: la succession d'une entreprise ne concerne pas seulement l'entreprise elle-même, mais a également des répercussions sur ses relations d'affaires (clients, fournisseurs etc.). Notre enquête le confirme: la plupart des PME se soucient des successions d'entreprise de leurs partenaires commerciaux. Seules 30\% des PME indiquent ne pas s'en préoccuper (figure 21). II s'agit avant tout de PME qui n'ont pas encore réfléchi non plus à leur propre succession. Mais bon nombre de PME s'informent activement de la situation auprès de leurs partenaires commerciaux (32\%) et/ou essaient déjà de nouer des contacts avec les successeurs potentiels (38\%). Ce faisant, elles espèrent en général poursuivre la relation d'affaires 
existante dans sa forme présente. Seules 13\% des entreprises tentent de formaliser la relation existante et de se couvrir par des accords écrits et des contrats. 13\% des PME déclarent en outre préparer des stratégies alternatives pour le cas où la transmission de l'entreprise du partenaire commercial échouerait. Une chose est claire: la succession d'une entreprise mobilise des ressources bien au-delà de l'entreprise elle-même.

\section{Figure 21}

\section{Quelle est votre position par rapport aux succes- sions d'entreprise de vos partenaires commerciaux?}

$\mathrm{n}=2063$ (plusieurs réponses possibles)

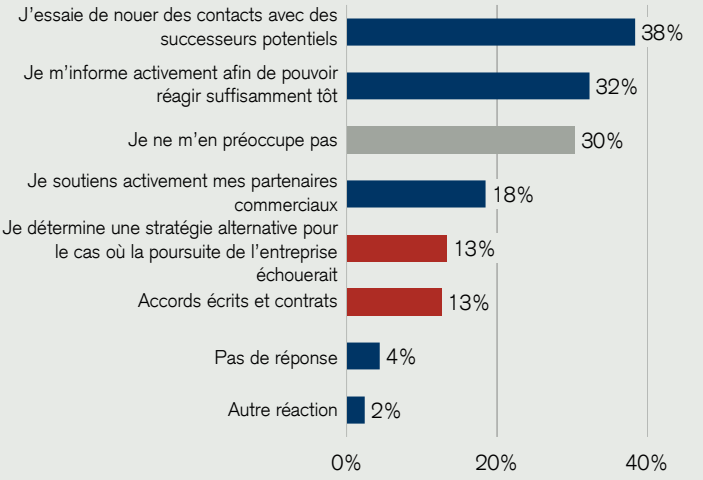

Source: Enquête PME Credit Suisse 2013

\section{Figure 22}

\section{D'après vous, quelle mesure pourrait le plus simpli- fier la succession d'entreprise?}

$n=523^{*}$ (une seule réponse possible)

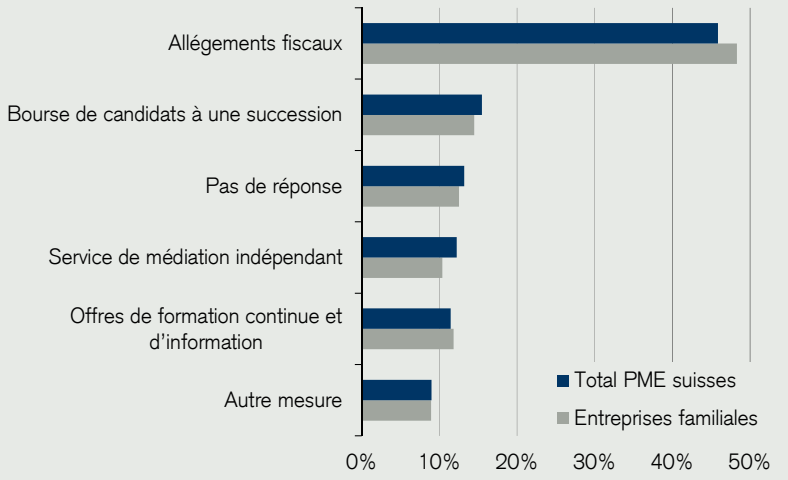

Source: Enquête PME Credit Suisse 2013; "réponse uniquement en cas de reprise/cession de l'entreprise survenue au cours des 10 dernières années
Des allégements fiscaux simplifieraient considérablement les successions

La fiscalité particulièrement handicapante pour les entreprises familiales
L'âge et la santé comme principaux motifs de transmission d'entreprises
Dans l'intérêt de l'économie, il est préférable de ne pas compliquer inutilement les successions d'entreprise. Lors de notre enquête, nous avons donc demandé à des entrepreneurs ayant repris une entreprise ces dix dernières années quelles mesures publiques ou extérieures aux entreprises pourraient faciliter la succession d'entreprise (figure 22). Une grande partie d'entre eux ont répondu que des allégements fiscaux faciliteraient particulièrement la transmission. Le manque d'information, l'absence de marché pour les repreneurs et le soutien insuffisant dans la résolution des conflits sont beaucoup moins souvent ressentis comme des obstacles.

Les entrepreneurs familiaux sont beaucoup plus nombreux à exprimer le souhait d'allègements fiscaux que les entrepreneurs non familiaux. Après l'imbroglio autour de la notion de «liquidation partielle indirecte», c'est désormais le dépôt de l'initiative pour un impôt sur les successions qui suscite les craintes et le mécontentement des entreprises familiales. Jusqu'à présent, l'impôt sur les successions est régi au niveau cantonal et il est quasi inexistant dans la plupart des cantons. La Suisse dispose ainsi d'un avantage de localisation non négligeable en comparaison internationale. En cas d'acceptation de l'initiative, les héritages à partir de 2 millions de francs seraient soumis rétroactivement à un impôt sur les successions s'élevant à $20 \%$. Cette rétroactivité est déconcertante, car elle remet en cause d'une manière générale les principes de sécurité du droit et de la planification. Eu égard aux règlements de succession en entreprise, I'initiative conduit à une restriction de la liberté d'action et de décision entrepreneuriale. Le patrimoine de l'entreprise constitue généralement un patrimoine familial illiquide. Dans la pratique, il faudrait donc s'inspirer des réglementations de pays comme l'Allemagne disposant d'un impôt sur les successions afin d'envisager la mise en place d'allègements fiscaux tels que des abattements et décotes ou des possibilités de report d'impôts avec extinction si l'activité est poursuivie pendant une période définie.

\section{Importance pour l'entrepreneur}

Pour l'écrasante majorité des entrepreneurs, la principale raison de se retirer de l'entreprise est la question de l'âge et de la santé (figure 23). D'autres motifs tels que le souhait de plus de temps libre ou la présence d'un successeur adéquat n'arrivent que très loin derrière. II est frap- 
L'importance de la succession d'entreprise culmine entre 60 et 65 ans pant de constater à quel point les motifs de succession sont homogènes quelle que soit la taille des entreprises. Certes, le souhait de plus de temps libre est cité par une proportion légèrement plus importante d'entrepreneurs comptant entre 10 et 49 collaborateurs. Mais les écarts ne sont pas significatifs. Cela montre une chose: beaucoup d'entrepreneurs suisses considèrent leur entreprise comme l'œuvre de leur vie. Ils s'y sont créé leur emploi à vie et ne souhaitent pas en partir.

II existe certes quelques jeunes entrepreneurs considérant le thème de la succession d'entreprise comme important pour eux. Mais ce n'est qu'à partir de l'âge de 50 ans qu'il prend une grande importance (figure 24). D'après notre sondage, c'est entre 60 et 65 ans que les entrepreneurs accordent la plus grande importance (par solde) à la succession d'entreprise. Dans I'ensemble, 44\% des PME considèrent ce thème comme important voire très important pour elles et $40 \%$ comme peu voire pas du tout important. $73 \%$ des entreprises ont déjà réfléchi au moins dans les grandes lignes à cette question.

\section{Figure 23}

\section{Quelle est votre raison principale de vous retirer de votre entreprise?}

Part des PME par classe de taille; $n=1507^{\star}$

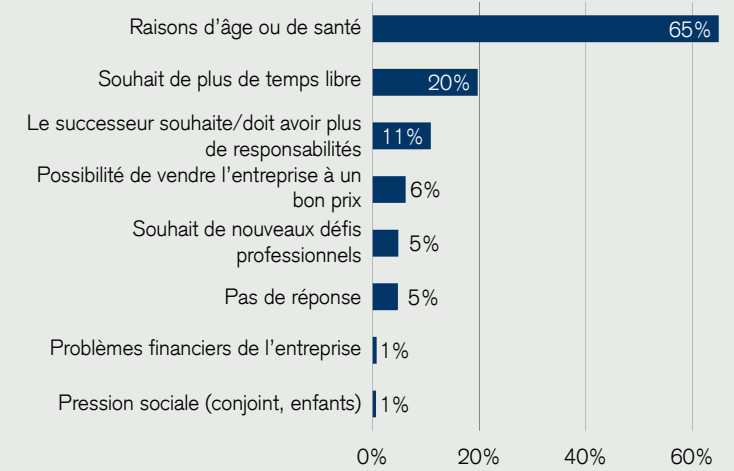

Source: Enquête PME Credit Suisse 2013; *réponse uniquement lorsque l'entrepreneur a déjà réfléchi à un règlement de succession

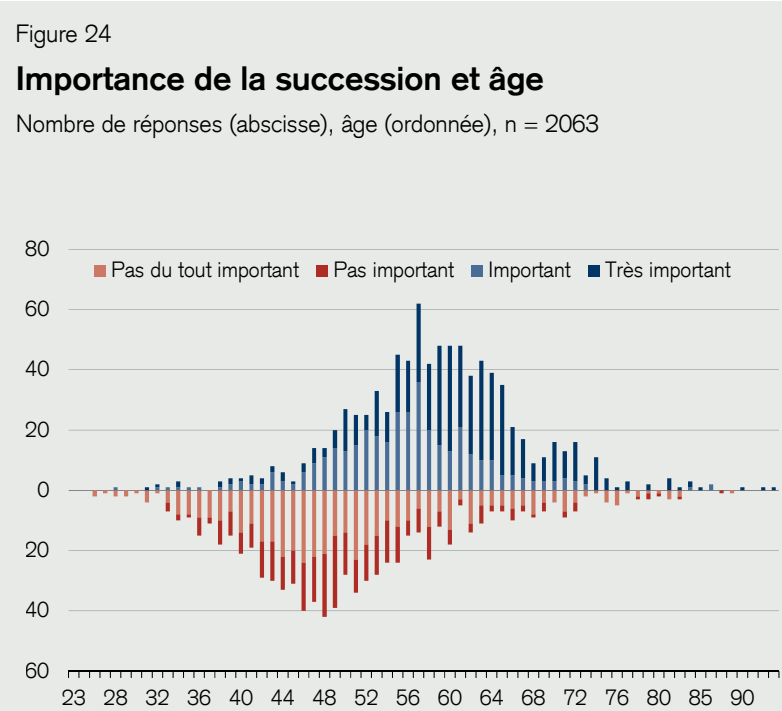

Source: Enquête PME Credit Suisse 2013
La prévoyance vieillesse amène les entrepreneurs à se pencher sur le thème de la succession

Les entrepreneurs suisses âgés de 55 ans en moyenne
Les entreprises familiales réfléchissent davantage au thème de la succession d'entreprise que les entreprises non familiales. Ceci s'explique notamment par le fait que l'entreprise fasse très souvent partie de la prévoyance vieillesse dans le cas des entreprises familiales. Les entrepreneurs dont la société fait partie de la prévoyance vieillesse se penchent plus souvent sur ce thème. D'après notre enquête, l'entreprise fait aujourd'hui partie de la prévoyance vieillesse de près de la moitié des entrepreneurs. II est intéressant de constater que la part d'entrepreneurs dont l'entreprise fait partie de la prévoyance vieillesse est significativement plus élevée chez les fondateurs que chez les non fondateurs.

\section{Démographie des entrepreneurs}

L'enquête PME de cette année nous permet de considérer un peu plus en détail la démographie générale des entrepreneurs et des entreprises. L'âge moyen des gérants de PME suisses est actuellement de 55 ans. Ils ont donc en moyenne pratiquement le même âge que leurs pairs des 100 plus grandes entreprises suisses. La répartition par âge des entrepreneurs de petites et moyennes entreprises suisses montre que l'entrée en entrepreneuriat se fait continuellement (figure 25). Jusqu'à l'âge de 50 ans environ, la part d'entrepreneurs augmente sans cesse. La phase de retrait commence ensuite vers 57 ans. Elle suit une évolution en grande partie symétrique à celle de la phase d'entrée et dure simplement un peu plus longtemps. En distinguant les types d'entreprises, on constate que les entrepreneurs familiaux sont tendanciellement un peu plus âgés que les entrepreneurs non familiaux (figure 26). Autrement dit: les entrepre- 
La population active plus jeune de 11 ans que les entrepreneurs neurs familiaux ont tendance à transmettre leur entreprise légèrement plus tard, ou les entreprises non familiales ont tendanciellement plus tôt la possibilité d'être reprises.

On peut également dresser des parallèles intéressants avec la démographie de la population active: I'âge moyen de la population active est inférieur de 11 ans à celui des entrepreneurs et s'élevait à 44 ans en 2009. L'activité de la population active et celle des entrepreneurs s'étendent sur une durée similaire, mais avec un décalage dans le temps. Les entrepreneurs restent beaucoup plus tard dans la vie active. L'arrivée à l'âge de la retraite est beaucoup moins considérée comme un motif de cessation de l'activité professionelle par les entrepreneurs que par la population active dans son ensemble.

\section{Figure 25}

\section{Démographie des entrepreneurs et de la population}

Age des entrepreneurs de PME en années, $n=2063$; population active (EPT) 2009, population constante 2011

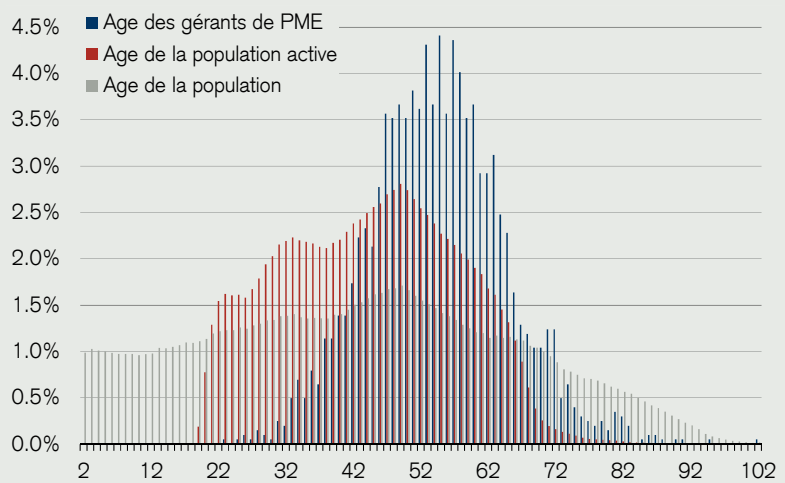

Source: Enquête PME Credit Suisse 2013, Office fédéral de la statistique

\section{Figure 26}

\section{Age des entrepreneurs familiaux et non familiaux}

Age du gérant en années; $n=1582$ (entreprises familiales), $n=431$ (entreprises non familiales)

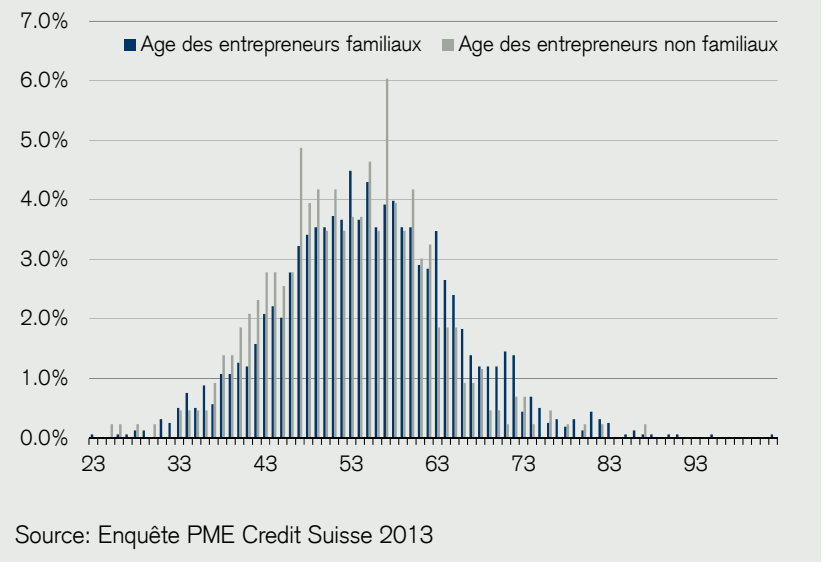

La transmission entraîne un rajeunissement de 24 ans

\section{L'âge de la direction ne varie quasiment pas en fonction de la branche, contrairement à l'âge de l'entreprise}

La succession d'entreprise entraîne généralement un rajeunissement de la direction (figure 27). Dans 4\% des cas seulement, l'âge du repreneur est supérieur à celui de son prédécesseur. La différence d'âge moyenne entre le repreneur et le prédécesseur est de 24 ans. Ainsi, dans le cadre de leur succession, les entreprises font un saut de moins d'une génération.

L'âge des gérants ne varie que très peu en fonction de la branche. II existe en revanche d'importantes différences concernant l'âge des entreprises. La PME moyenne a été fondée en 1968. Les plus vieilles PME sont en moyenne celles de l'industrie traditionnelle (année moyenne de fondation: 1950) (figure 28). Les plus jeunes sont les entreprises du secteur des TIC (année moyenne de fondation: 1988). Ceci a bien entendu plusieurs raisons: certaines technologies sont tout simplement récentes. Ainsi, il n'y avait quasiment pas d'entreprises de TIC avant 1980. Les perspectives de succès et donc les taux de faillite diffèrent suivant les branches, qui n'offrent pas toutes les mêmes chances de transmettre l'entreprise à la génération familiale suivante. Par exemple, d'après notre étude, les PME de l'industrie traditionnelle en sont plus souvent déjà à la deuxième ou troisième génération que les entreprises de la branche du tourisme et des loisirs. 


\section{Figure 27}

\section{Age du gérant et du prédécesseur}

Age en années, $\mathrm{n}=2018$ (gérants), $\mathrm{n}=1006$ (prédécesseurs), moyennes mobiles sur 5 ans

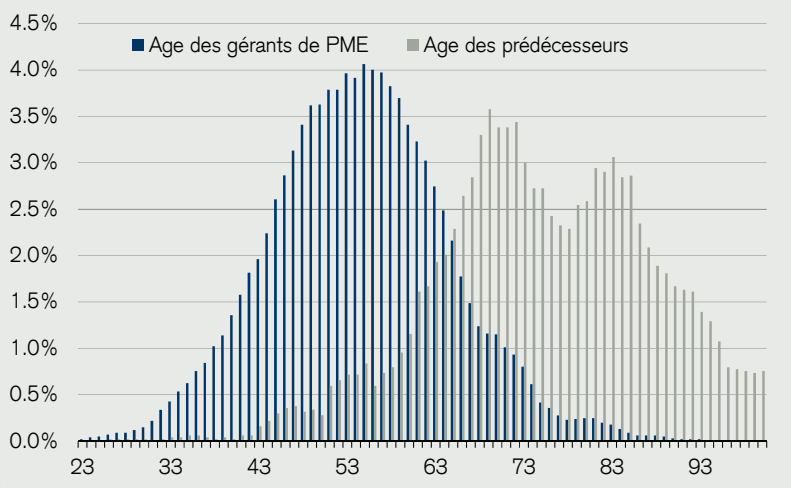

Source: Enquête PME Credit Suisse 2013
Figure 28

\section{Démographie des PME par branche}

Age moyen des gérants et des PME par branche; $n=2063$

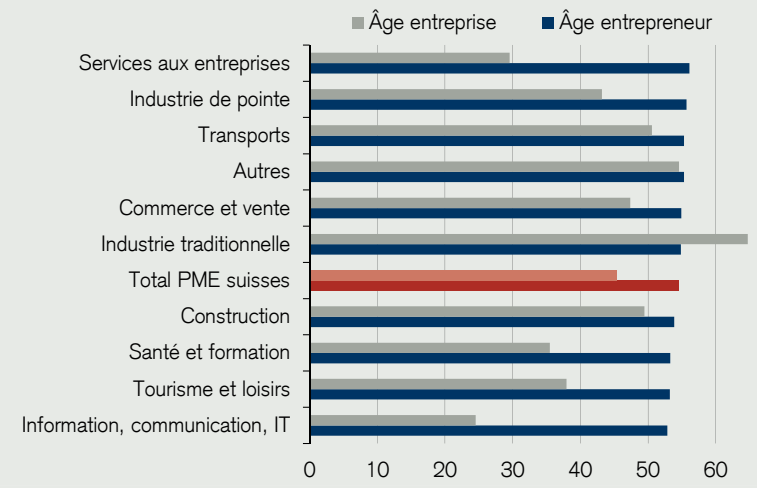

Source: Enquête PME Credit Suisse 2013
Entreprises familiales particulièrement concernées par la succession

$78 \%$ des PME suisses sont aujourd'hui des entreprises familiales

Influence opérationnelle et stratégique plus importante que la propriété dans la perception subjective

\section{Entreprises familiales}

Les entreprises familiales sont particulièrement concernées par le thème de la succession d'entreprise. Dans ces entreprises, les mondes du travail et de la famille sont étroitement liés. Lorsque l'entrepreneur se retire, l'entreprise et la famille doivent donc se redéfinir indépendamment l'une de l'autre. Ceci est d'autant plus compliqué qu'il existe bien souvent d'importants liens financiers entre l'entreprise et la famille dans les entreprises familiales. L'entreprise constitue une part significative de la fortune familiale et d'après notre enquête, elle fait aussi plus souvent partie de la prévoyance vieillesse que dans les entreprises non familiales.

On estime que la part d'entreprises familiales dans le monde est comprise entre 60 et 90\%. Pour la Suisse, elle a été évaluée à $88 \%$ en $2004 .{ }^{17}$ Notre enquête montre qu'aujourd'hui, si I'on utilise la même définition des entreprises familiales que celle généralement établie dans la littérature de référence ${ }^{18}$, la part des entreprises familiales n'est plus que de $78 \%$. La baisse est encore plus forte si I'on interroge directement les PME: à peine $70 \%$ se définissent comme des entreprises familiales.

Les signes caractéristiques indiquant qu'une entreprise est une entreprise familiale sont la détention par la famille d'une part importante de l'entreprise ainsi que la présence de membres de la famille au sein de l'organe de surveillance (conseil d'administration) et/ou de la direction. L'examen détaillé de ces trois facteurs montre que les familles jouent surtout un rôle prépondérant en termes de propriété de l'entreprise. Plus de $65 \%$ des entreprises sont détenues à $100 \%$ dans la famille et $75 \%$ des entreprises le sont à plus de $75 \%$ (figure 29 ). Dans un peu plus des deux tiers des PME, la direction opérationnelle est contrôlée à plus de $50 \%$ par la famille, et dans $54 \%$ des PME, la direction stratégique se compose majoritairement de membres de la famille. Pour ce qui est des PME se considérant subjectivement comme des entreprises familiales, $88 \%$ d'entre elles sont détenues à plus de $75 \%$ dans la famille, plus de $60 \%$ ont un organe de surveillance majoritairement familial et plus de $74 \%$ une direction majoritairement familiale. Ainsi, les principaux critères du point de vue des entrepreneurs semblent être un rôle prépondérant de la famille en termes de propriété de l'entreprise ainsi qu'une forte influence au sein de la direction.

17 Frey, Halter, Zellweger (2004): Bedeutung und Struktur von Familienunternehmen in der Schweiz.

18 Nous appelons entreprises familiales les entreprises où il existe une influence familiale substantielle («substantial family influence»). Nous considérons qu'une telle influence familiale substantielle existe lorsque la somme de la part de propriété détenue par la famille, la part de membres de la direction issus de la famille et la part de membres de I'organe de surveillance issus de la famille est supérieure à 100\% (cf. Halter/Schröder 2010). Pour les entreprises ne pouvant pas être identifiées clairement comme familiales ou non familiales du fait de données manquantes, nous reprenons l'auto-définition subjective de ces entreprises qui leur a également été demandée dans l'enquête. 
Figure 29

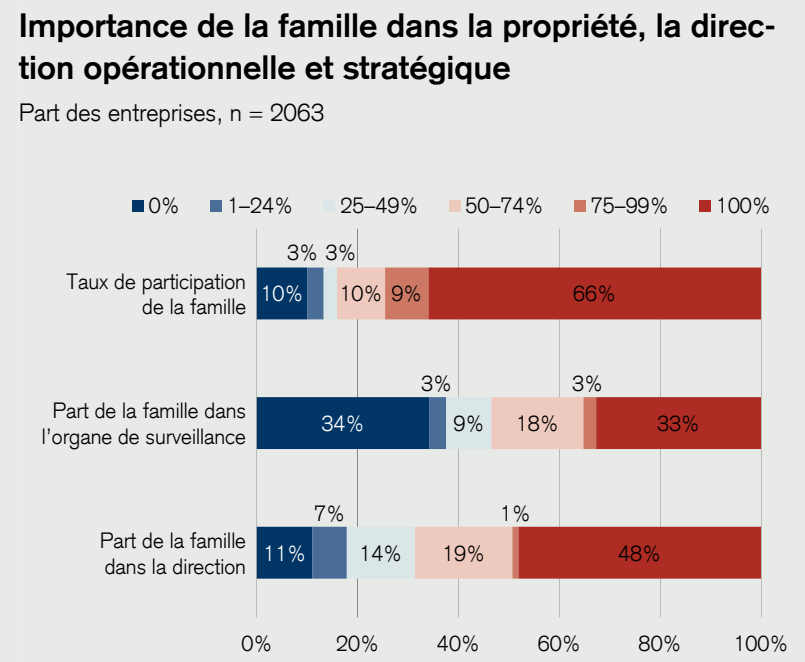

Source: Enquête PME Credit Suisse 2013
Figure 30

\section{Part d'entreprises familiales par branche*}

Part des réponses, $n=2063$

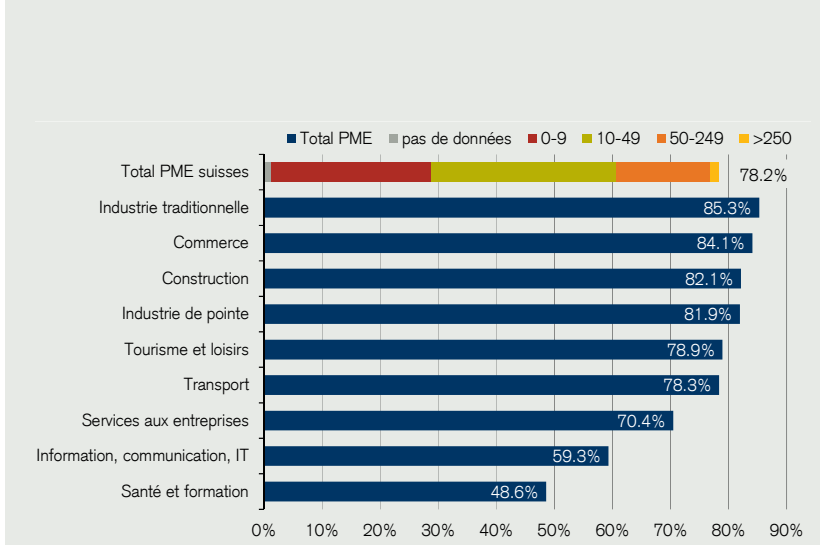

Source: Enquête PME Credit Suisse 2013; *selon le critère de la «substantial family influence», voir note de bas de page 18

\section{Net recul ces neuf dernières années}

Les entreprises familiales fortement représentées dans toutes les branches et classes de taille

Grand souhait de transmission à la famille
Le net recul du taux d'entreprises familiales en moins de dix ans est quelque peu surprenant. II peut notamment s'expliquer par la baisse du nombre de reprises intrafamiliales. La part d'entreprises familiales est même significativement plus élevée parmi les entreprises dirigées par leur fondateur que chez celles dirigées par un non-fondateur. L'analyse des entreprises par période de fondation montre en outre que le taux d'entreprises familiales a toujours été sujet à des fluctuations. C'est pourquoi il ne faut pas non plus partir du principe que le taux d'entreprises familiales va continuer de baisser dans le futur - bien au contraire. Les caractéristiques typiques du modèle commercial classique des entreprises familiales, à savoir la focalisation sur le long terme, la forte orientation clientèle, la culture d'entreprise respectueuse des collaborateurs et l'importance d'une gérance durable, ont gagné en attractivité ces derniers temps, comme le montre une étude du Credit Suisse Research Institute. ${ }^{19}$

Abstraction faite des similitudes liées à leur modèle commercial, les entreprises familiales sont aussi diverses que l'est l'économie elle-même. La part d'entreprises familiales diminue certes à mesure qu'augmente la taille des entreprises, mais on trouve de nombreuses entreprises familiales y compris parmi les grandes entreprises cotées en bourse. En ce qui concerne la répartition par branche, on constate que les entreprises familiales sont particulièrement bien représentées dans les branches de l'industrie traditionnelle, du commerce, de la construction ainsi que du tourisme et des loisirs (figure 30). En revanche, les prestataires de services aux entreprises, les entreprises du secteur des TIC et celles des domaines de la formation et de la santé sont beaucoup plus rarement détenues par des familles.

En matière de succession, les entreprises familiales du monde entier ont un point commun: leur grand souhait de transmettre l'entreprise au sein de la famille à la génération suivante. ${ }^{20} \mathrm{La}$ famille joue souvent un rôle extrêmement important de pourvoyeur de ressources financières lors de la fondation d'une entreprise. Elle occupe de nouveau une place centrale lors de la succession d'entreprise. Mais la transmission d'entreprise au sein de la famille recèle un fort potentiel de risques et de conflits: disputes concernant la répartition des pouvoirs, peur de perte de pouvoir, inadéquation des successeurs, inertie grandissante et désaccords sur l'orientation stratégique. Ainsi, il n'est pas rare que la famille coure le risque d'échouer voire d'éclater avec l'entreprise lors de la succession. Une planification successorale systématique s'impose donc en amont. 


\section{Etat des lieux: enseignements et recommandations pour la pratique}

- La succession d'entreprise est un enjeu stratégique pour l'entreprise et la famille. Prenez le temps nécessaire. Une succession qui échoue détruit de la valeur.

- 22\% des PME suisses sont confrontées à la même question, vous n'êtes pas seul(e) ni un cas isolé. Echangez avec des pairs.

- Votre disposition à transmettre l'entreprise est la première condition pour une succession réussie.

- L'aptitude de votre entreprise à faire l'objet d'une reprise (dépendant p. ex. de la branche, la stratégie de propriétaire) est la deuxième condition pour une succession réussie.

- Vos partenaires commerciaux souhaitent savoir si votre entreprise restera à l'avenir un partenaire valable. Pensez à informer vos partenaires commerciaux et à leur présenter votre successeur.

- L'âge et la santé sont les deux principaux motifs de règlement de succession. Avec l'âge, le risque d'événements imprévus augmente. II est recommandé de réfléchir à un "plan d'urgence» et de se préparer à un tel scénario.

- L'optimisation fiscale et la prévoyance ne sont pas des problématiques de court terme. Elles doivent être coordonnées entre elles et planifiées suffisamment tôt.

\section{Règlements de successions: souhaits et réalité}

\section{Les entreprises familiales souhaitent transmettre à la famille, les entreprises non familiales à des collabora- teurs}

Vendre l'entreprise, garder la direction

\section{Point de vue des cédants}

La formulation spéciale de l'enquête avec d'une part des questions s'adressant aux PME en général et d'autre part des questions aux repreneurs d'entreprises nous permet de confronter les projets et les souhaits de transmission des entrepreneurs avec la réalité. Lorsqu'on demande aux entrepreneurs suisses qui doit reprendre un jour leur entreprise, on obtient les résultats suivants: les entreprises familiales préfèrent généralement être transmises au sein de la famille (family buy-out, FBO) ${ }^{21}$ tandis que les entreprises non familiales souhaiteraient idéalement être reprises par leurs collaborateurs (management buy-out, $\mathrm{MBO}^{22}$ ) (figure 31). C'est en particulier la direction de l'entreprise qu'elles souhaitent garder autant que possible au sein de la famille ou respectivement de l'entreprise.

En ce qui concerne la transmission de la propriété de l'entreprise, les entreprises familiales comme non familiales semblent nettement plus enclines à une solution extérieure à l'entreprise que pour la transmission de la direction. Certes, les entreprises familiales préféreraient en premier lieu transmettre également la propriété de l'entreprise au sein de la famille. Mais leur deuxième solution favorite est le management buy-in $(\mathrm{MBI})^{23}$ impliquant des personnes extérieures à l'entreprise, qu'elles préfèrent de loin au management buy-out (MBO). Le constat est similaire chez les entreprises non familiales: la préférence pour le MBI l'emporte très légèrement en ce qui concerne la transmission de la propriété. Ces préférences différentes pour la transmission de la propriété et de la direction de l'entreprise reflètent probablement le tiraillement des entrepreneurs entre, d'une part, leur souhait de garantir la continuité de l'entreprise en la transmettant à une personne familière et, d'autre part, leur volonté d'obtenir le meilleur prix pour la propriété.

21 Family buy-out (FBO): Vente de l'entreprise à un ou plusieurs membres de la famille.

22 Management buy-out (MBO): Vente de l'entreprise à un ou plusieurs collaborateurs dirigeants.

23 Management buy-in (MBI): Vente de l'entreprise à une personne physique ou morale n'ayant initialement aucun lien avec l'entreprise et ses parties prenantes. 
Figure 31

\section{Que doit-il advenir de la propriété/la direction de votre entreprise?}

Part des réponses, $\mathrm{n}=1507$ (plusieurs réponses possibles)

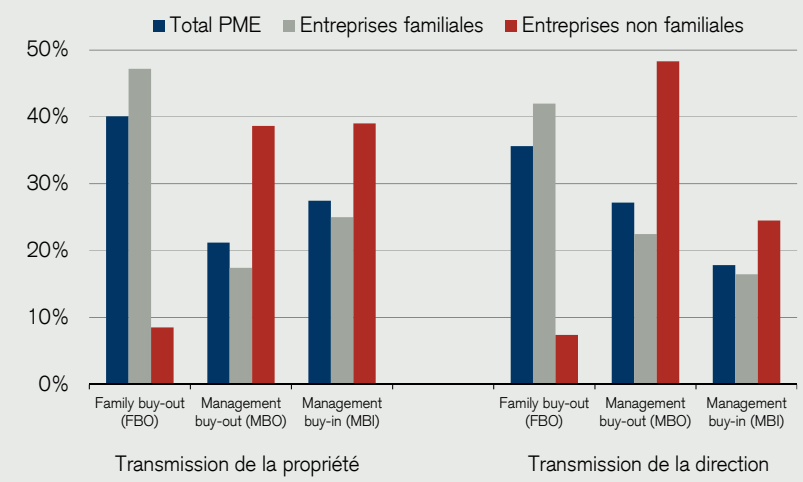

Source: Enquête PME Credit Suisse 2013
Figure 32

\section{Transmission de la propriété selon la classe de taille d'entreprise}

Part de règlements de succession prévus (transmission de la propriété) par classe de taille en 2013, évolution par rapport à 2009 en points de pourcentage; $n=2063$ (2013), $n=931$ (2009)

\begin{tabular}{rccccc}
$\begin{array}{r}\text { Taille de } \\
\text { l'entreprise }\end{array}$ & $\begin{array}{c}\text { Family } \\
\text { buy-out }\end{array}$ & \multicolumn{2}{c}{$\begin{array}{c}\text { Management } \\
\text { buy-out }\end{array}$} & \multicolumn{2}{c}{$\begin{array}{c}\text { Management } \\
\text { buy-in }\end{array}$} \\
$0-9$ & $47 \%(+1 \%)$ & $24 \%$ & $(+3 \%)$ & $29 \%$ & $(-4 \%)$ \\
$10-49$ & $44 \%(-1 \%)$ & $27 \%$ & $(-2 \%)$ & $29 \%$ & $(+4 \%)$ \\
$50-249$ & $54 \%(+1 \%)$ & $23 \%$ & $(-5 \%)$ & $23 \%$ & $(+3 \%)$
\end{tabular}

Source: Enquête PME Credit Suisse 2013
Progression générale des successions extérieures à l'entreprise

\section{Micro-entreprises scep- tiques à l'égard des trans- missions à l'extérieur de l'entreprise}

Grande importance de la liberté de choix des enfants

Les entrepreneurs suisses encouragent leurs amis et enfants à devenir entrepreneurs
Les souhaits de transmission sont globalement similaires dans toutes les classes de taille d'entreprise (figure 32). Comparativement à notre enquête précédente, on constate une légère augmentation de la part de transmissions extérieures à l'entreprise (MBI) chez les petites et moyennes entreprises. Celle-ci s'est surtout faite au détriment des transmissions à des collaborateurs (MBO). Ceci peut avoir plusieurs raisons: d'une part, les opportunités de transmissions (ventes) à des personnes physiques ou morales extérieures à l'entreprise sont peut-être plus nombreuses aujourd'hui. D'autre part, et dans la lignée de cette première hypothèse, il se pourrait également que les entrepreneurs soient désormais moins enclins à consentir aux collaborateurs un rabais conséquent sur la valeur de marché de l'entreprise. Cette seconde hypothèse est confortée par le fait que, malgré la préférence croissante pour la transmission de propriété à l'extérieur de l'entreprise, on constate une hausse des souhaits de transmission à des membres de la famille, tout au moins pour les entreprises de 50 à 249 collaborateurs. Une autre explication possible réside dans le faible niveau actuel des taux d'intérêt et des rendements du marché des capitaux, qui rend les entreprises plus attractives en tant qu'investissement - pour l'entrepreneur lui-même ou pour des personnes extérieures à l'entreprise. Toutefois, les écarts sont faibles et on ne peut pas complètement exclure d'éventuels effets induits par l'échantillon.

Il est intéressant de constater une tendance opposée chez les micro-entreprises. La part des transmissions à l'extérieur de la famille s'est reportée sur les transmissions internes à l'entreprise $(\mathrm{MBO})$ au détriment des transmissions à l'extérieur de l'entreprise $(\mathrm{MBI})$. Parallèlement, les souhaits de transmission à des membres de la famille ont augmenté par rapport à 2009.

Le family buy-out prédomine dans les projets des entrepreneurs. Mais ce souhait ne se réalise pas toujours. Lorsque la direction ne peut être transmise aux enfants, c'est généralement parce que ces derniers ne le souhaitent pas (figure 34). Les entrepreneurs suisses semblent respecter ces souhaits de leurs enfants et accorder une grande importance à leur liberté de choix professionnel. Sans doute ont-ils conscience qu'en cas de décision contrainte ou d'inadéquation des successeurs, des conflits surviendront tôt ou tard. Toutefois, il se pourrait bien que la progéniture des entrepreneurs familiaux ressente indirectement une certaine pression pour reprendre l'entreprise familiale, comme le montrent la figure 37 et la figure 38. Les entrepreneurs familiaux déclarent par ailleurs un peu plus souvent encourager leurs enfants et amis à devenir entrepreneurs que les entrepreneurs non-familiaux.

D'une manière générale, neuf entrepreneurs sur dix encourageraient leurs amis et enfants à devenir entrepreneurs (figure 33). Ceci montre que l'écrasante majorité des entrepreneurs a une image positive de l'entrepreneuriat. Celle-ci est sans doute liée au fait que les entrepreneurs puissent souvent créer leur emploi à vie dans leur entreprise et indique également que l'entrepreneuriat jouit en Suisse d'une bonne et haute considération sociale. 
Figure 33

\section{Encourageriez-vous vos enfants ou vos amis à devenir entrepreneurs?}

Part des réponses, $n=1507$

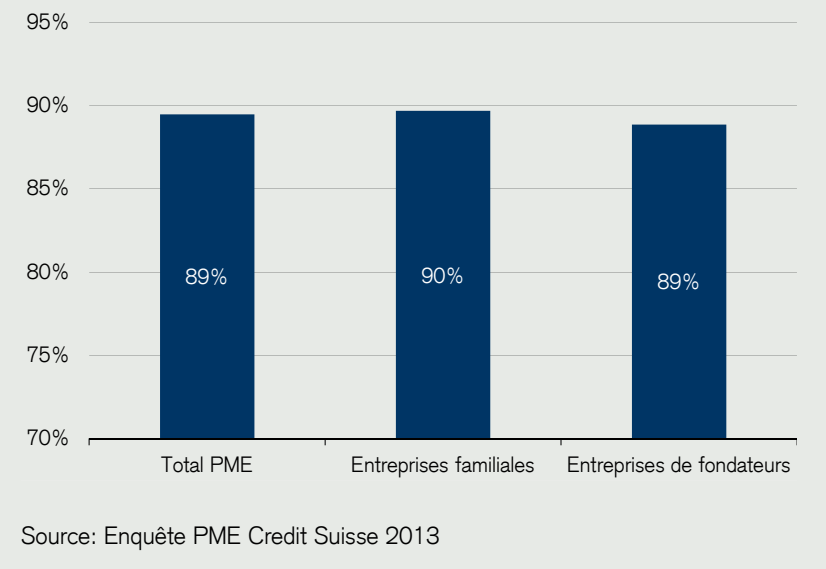

Figure 34

\section{Si vous ne souhaitez pas transmettre la direction à vos enfants, pour quelle raison?}

Part des réponses, $n=1507$

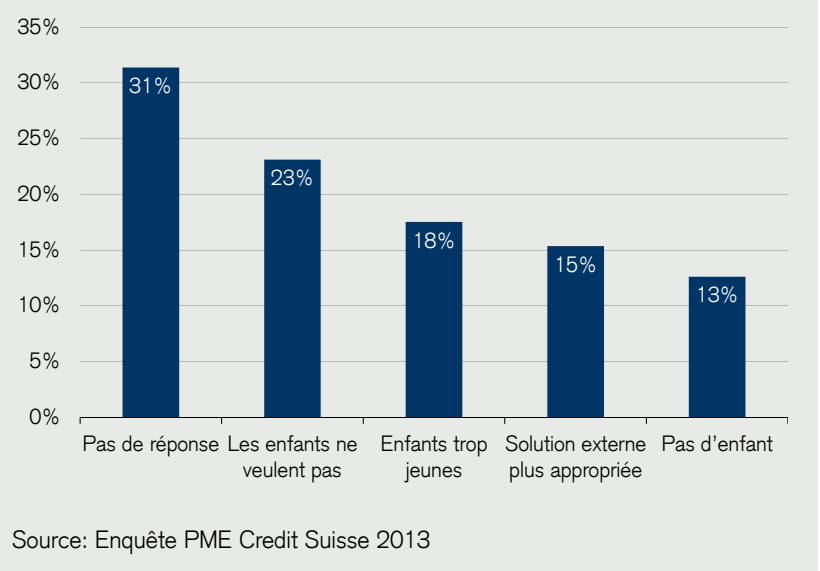

La réalité est légèrement différente

\section{Les FBO et MBI sont au coude à coude parmi les solutions de succession retenues}

Des reprises intrafamiliales stables et conformes aux prévisions des PME

\author{
Le MBI plus utilisé que \\ prévu, le MBO pâtit de sa \\ mise en œuvre
}

\section{Point de vue des repreneurs}

La partie précédente de l'étude s'est focalisée sur la succession d'entreprise en général et sur le point de vue des cédants en particulier. Dans la partie qui suit, nous nous intéresserons avant tout au point de vue des repreneurs et donc aux successions d'entreprise réalisées. Pour cela, nous ne prendrons en considération que les réponses des gérants ayant repris l'entreprise de leur prédécesseur au cours des dix dernières années. II en résulte un échantillon de 523 réponses au maximum.

Concernant les successions d'entreprise réalisées, examinons d'abord quelle était la relation entre le gérant actuel et son prédécesseur. II s'avère que près de $40 \%$ des entrepreneurs ont repris l'entreprise de leur famille (FBO), tandis qu'environ 40\% ont réalisé un management buyin (MBI) et environ $20 \%$ seulement un management buy-out (MBO) (figure 35 ).

Avec $40 \%$, la part de FBO reste quasi constante par rapport à l'étude de 2009. La succession intrafamiliale est donc actuellement choisie dans près de $40 \%$ des règlements de successions. Il est en revanche assez surprenant de constater que le $\mathrm{MBI}$ a été utilisé beaucoup plus souvent que lors de l'enquête précédente, mais aussi beaucoup plus souvent que dans les intentions exprimées ci-avant par les futurs vendeurs (27\%).

C'est pour le $\mathrm{MBI}$ que l'écart entre les souhaits exprimés et la réalité est le plus grand. Bon nombre d'entrepreneurs n'ayant pas de souhait concret quant à l'identité de leur futur repreneur cèdent finalement l'entreprise à des personnes extérieures. Le MBO est le seul des trois règlements de succession à être réalisé moins souvent que prévu. La pratique montre que beaucoup d'entrepreneurs songent à leurs collaborateurs lorsqu'aucun membre de leur famille ne veut ou ne peut prendre la relève. De prime abord, les collaborateurs se montrent eux-aussi intéressés par cette option. Mais leur disposition à prendre des risques s'avère souvent insuffisante pour sauter le pas. Les entreprises finissent donc par chercher des repreneurs en dehors de l'entreprise. 


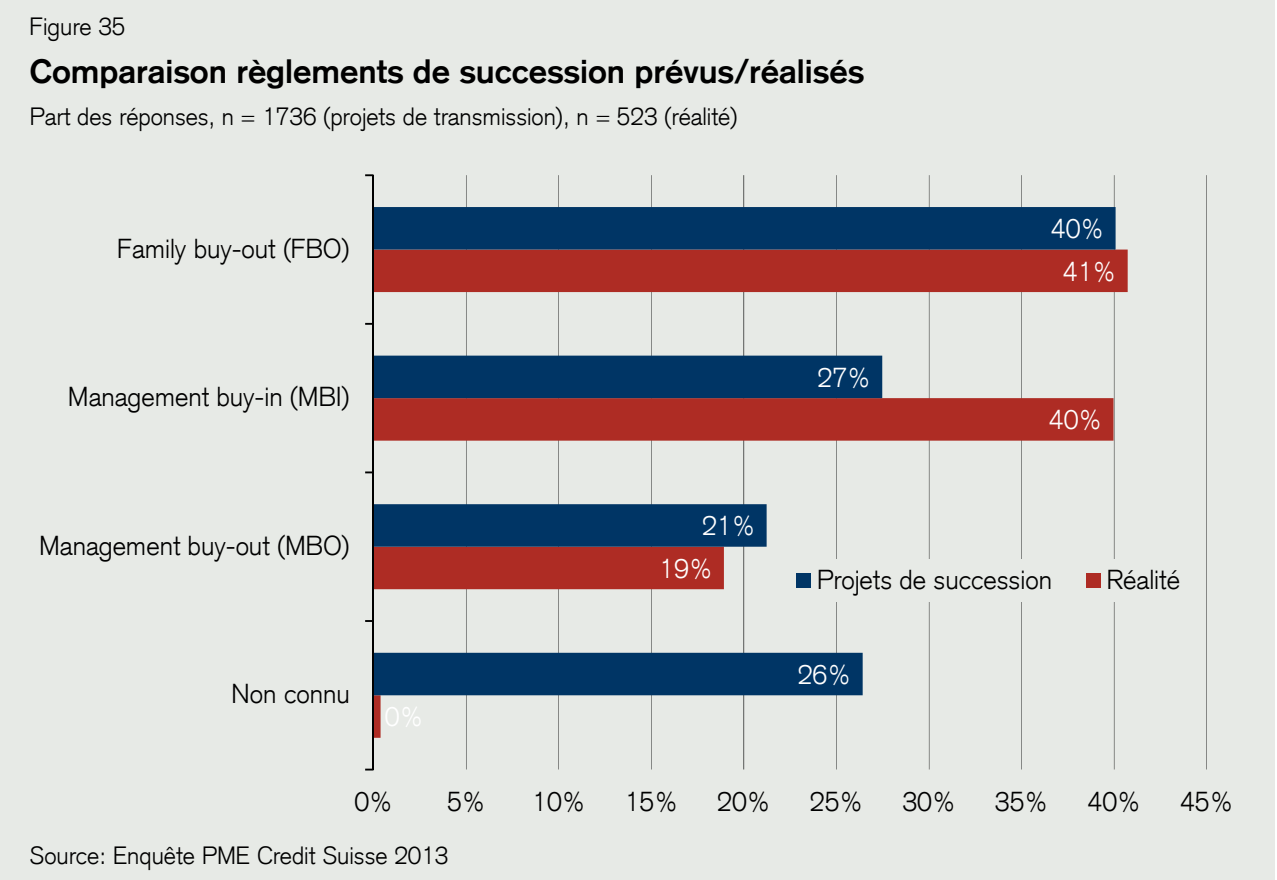

Dans les familles, ce sont les fils qui prennent la relève

Seule la moitié des transmissions externes sont des MBI classiques

Les motivations des vendeurs correspondent à la perception des repreneurs

L'épanouissement personnel est le principal motif de reprise
Une analyse plus précise des FBO montre que les transmissions se font en grande partie de parents à enfant (83\% des $\mathrm{FBO})$. Dans la très grande majorité de ces cas, l'entreprise a été transmise à un fils et dans seulement 15 cas sur 178 à une fille. Par ailleurs, seule une part infime des entreprises a été reprise par des conjoints ( $2 \%$ des FBO).

Une analyse différenciée des $\mathrm{MBI}$ montre que dans près de $25 \%$ des cas de $\mathrm{MBI}$, le repreneur était un ami ou une connaissance du vendeur avant la transmission. 25\% des repreneurs entretenaient quant à eux une relation d'affaires avec l'entreprise (p. ex. en tant que client ou fournisseur). Ces données montrent que l'environnement extérieur à la famille et à l'entreprise de l'entrepreneur joue un grand rôle dans le contexte d'une succession. Les 50\% restants ont indiqué n'avoir entretenu aucune des relations citées avec le vendeur avant la reprise. II s'agit donc là de cas de $\mathrm{MBI}$ «classiques» où le repreneur cherche activement un "objet» à reprendre, par exemple via des annonces ou des intermédiaires.

Un engagement entrepreneurial se fonde généralement sur des motivations, souhaits et objectifs divers. Nous nous intéresserons d'abord à la génération des vendeurs. Puis dans un second temps, nous nous pencherons sur celle des repreneurs. En ce qui concerne les successions d'entreprise réalisées, nous avons demandé aux repreneurs quelles étaient rétrospectivement les motivations ayant conduit leurs prédécesseurs à vendre leurs entreprises. Le résultat est très proche des motivations exprimées par les entrepreneurs envisageant de transmettre leur entreprise (voir à ce sujet figure 36 et figure 23). Dans 50\% des successions d'entreprise, l'âge et la santé étaient la principale raison. En deuxième et troisième position, on trouve le souhait de plus de temps libre et les problèmes financiers de l'entreprise. Le souhait de nouveaux défis professionnels et la pression sociale jouent en revanche un rôle subalterne.

Du point de vue des repreneurs, ce sont surtout leurs motivations pour la reprise qui nous intéressent. La possibilité d'épanouissement personnel est de loin celle ayant joué le plus grand rôle, avant même l'attrait financier. Ce résultat reflète une tendance sociétale généralisée sous nos tropiques. Dans un environnement macro-économique où, d'une part, les marchés du travail fonctionnent, le chômage est faible et la situation sociopolitique est stable et, d'autre part, la société est tournée vers la consommation et les loisirs, ce ne sont pas les motivations financières qui poussent en premier lieu les jeunes à devenir entrepreneurs. ${ }^{24}$ Ceux dont I'unique but est de gagner rapidement de l'argent ne se lancent pas dans une PME. 
Figure 36

\section{Quelle était la principale motivation du prédéces- seur pour la transmission?}

Part des réponses, $n=523$ (plusieurs réponses possibles)

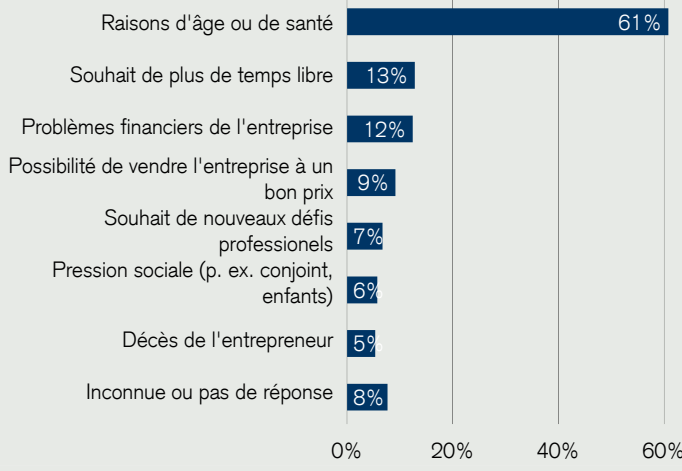

Source: Enquête PME Credit Suisse 2013
Figure 37

Quelle a été votre principale motivation pour la reprise de l'entreprise?

Part des réponses, $n=523$ (plusieurs réponses possibles)

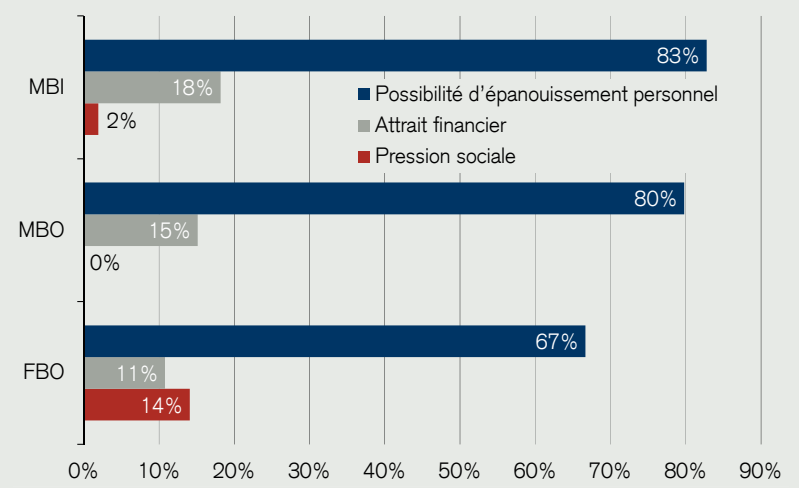

Source: Enquête PME Credit Suisse 2013
Une pression sociale larvée

La responsabilité sociale comme motif fréquent pour la reprise
La différenciation des FBO, MBO et MBI montre que c'est surtout dans les cas de MBO et MBI que la possibilité d'épanouissement personnel constitue la principale motivation (figure 37). Ces résultats montrent une fois de plus que les repreneurs de PME ne s'intéressent généralement pas qu'aux profits financiers escomptés. Comme on pouvait s'y attendre, c'est dans les cas de $\mathrm{MBI}$ que l'attrait financier a la plus grande importance et dans les cas de FBO que son importance est la plus faible. Même si la possibilité d'épanouissement personnel joue un rôle central dans les FBO, on ne peut pas nier l'existence d'une certaine pression sociale pour cette option de succession (dans environ une transmission intrafamiliale sur sept). Cette pression sociale est encore plus marquée lorsqu'on analyse qualitativement les réponses à la question ouverte (figure 38).

\section{Figure 38 \\ Quelle a été votre principale motivation pour la reprise de l'entreprise? \\ Evaluation qualitative de la question ouverte}

«Attachement à l'entreprise; poursuite de l'entreprise; la volonté générale d'être entrepreneur; les collaborateurs; le souhait d'assurer l'avenir de l'entreprise; conscience du devoir, responsabilité; tradition, vocation; indépendance; la création d'emplois; défi professionnel; le sauvetage du patrimoine familial; chômage; héritage; éviter les frais de liquidation; responsabilité sociale; décès du conjoint; attaque cérébrale du père; garant de l'héritage de mon père.»

Source: Enquête PME Credit Suisse 2013

Les réponses à la question ouverte montrent que c'est souvent la poursuite de la tradition et de l'entreprise familiale qui motive avant tout la reprise. Dans ces cas, la reprise de la direction de l'entreprise n'est manifestement pas ressentie comme le résultat d'une pression sociale. La motivation réside davantage dans la tradition, la conscience du devoir et la fierté. Un autre aspect essentiel ressortant des réponses libres est le thème de la responsabilité sociale. Plusieurs entrepreneurs indiquent avoir repris l'entreprise pour éviter une fermeture de celle-ci voire pour créer des postes de travail supplémentaires. Les réponses libres nous montrent également que plusieurs entrepreneurs ont pris la tête de l'entreprise sans vraiment y être préparés, p. ex. lors du décès du prédécesseur. On ne constate pas de différences significatives suivant la taille des entreprises. 


\section{Souhaits et réalité: enseignements et recommandations pour la pratique}

- Concernant les options de succession, il faut impérativement réfléchir en termes de scénarios. Rien ne garantit qu'un projet déterminé pourra être réalisé.

- En Suisse, il n'y a rien d'évident à ce que les enfants reprennent l'entreprise familiale.

- Pour les micro-entreprises, il est parfois difficile de trouver un acheteur ou successeur. La cessation d'activité volontaire peut ou doit être une option stratégique.

- Les motivations pour la reprise d'une entreprise sont multiples et variées. Elles doivent être appréhendées au cas par cas. La compréhension des raisons motivant la cession et la reprise d'une entreprise facilite le processus de rapprochement entre les prédécesseurs et les successeurs, et donc la transmission d'entreprise.

\section{Processus de transmission}

\section{Sélection des candidats et rôle de l'organe de surveillance}

La décision stratégique de la transmission ne dépend qu'en partie du conseil de surveillance

Les critères de gouvernance des grandes entreprises ne sont pas transposables directement aux PME
Les organes de surveillance comme le conseil d'administration ou le comité consultatif sont importants pour la prise de décisions stratégiques. Ce qui constitue une obligation pour la société anonyme est juridiquement facultatif pour les autres formes de sociétés. La succession d'entreprise est indiscutablement une problématique stratégique majeure, c'est pourquoi l'influence du conseil d'administration/du comité consultatif/de l'organe de surveillance lors du choix du candidat à la succession nous intéresse. Dans l'ensemble, on constate que le droit d'intervention de l'organe de surveillance est très variable (figure 39). Alors que celui-ci décide seul dans $40 \%$ des cas, il ne prend pas du tout part à la décision dans 30\% des cas. En détaillant les résultats par type de transmission, on constate comme on pouvait s'y attendre que l'organe de surveillance joue un rôle beaucoup moins important dans les FBO que dans les $\mathrm{MBO}$ ou MBI.

L'influence du conseil d'administration est particulièrement forte dans les petites et moyennes entreprises, tandis qu'il ne joue aucun rôle dans près de la moitié des micro-entreprises. II est intéressant de constater qu'il n'y a presque pas de différence entre les petites et les moyennes entreprises au sujet de l'influence de l'organe de surveillance. En particulier dans le monde des PME familiales, le thème de la gouvernance d'entreprise peut ou doit être considéré avec prudence. Les critères ne sont pas les mêmes que pour les grandes entreprises cotées en bourse, car dans le cas des PME, les propriétaires sont la plupart du temps également dirigeants. Vu sous cet angle, l'administrateur externe joue avant tout le rôle de sparring partner pour l'entrepreneur et propriétaire. Sa fonction décisionnelle est beaucoup moins marquée. On peut supposer que les propriétaires imposent en général plus facilement leur point de vue que les administrateurs externes.

\section{Figure 39}

Rôle du conseil d'administration dans la décision de succession

Part des réponses, $n=410$

\begin{tabular}{lcccc} 
& Total & Family buy-out & Management buy-out & Management buy-in \\
\hline Aucun rôle & $30 \%$ & $37 \%$ & $24 \%$ & $25 \%$ \\
Décision commune & $23 \%$ & $21 \%$ & $24 \%$ & $25 \%$ \\
Seul décideur & $40 \%$ & $37 \%$ & $42 \%$ & $43 \%$ \\
$\begin{array}{l}\text { Non connu/pas de } \\
\text { réponse }\end{array}$ & $7 \%$ & $5 \%$ & $10 \%$ & $7 \%$
\end{tabular}

Source: Enquête PME Credit Suisse 2013 
Les entreprises familiales sont souvent transmises à des "dauphins"

Un successeur extérieur à la famille sur quatre a dû s'imposer face à d'autres candidats extérieurs

Le réseau comme important vivier de candidats

Il s'écoule du temps entre le premier contact et le transfert de responsabilité

Une durée de transmission comprise en moyenne entre 1,6 et 6,5 ans
Dans la succession d'entreprise, il n'y a pas que les aspects décisionnels qui soient intéressants: on peut également se demander si l'entreprise avait le choix entre un ou plusieurs candidats. Naturellement, il est difficile pour le successeur de répondre à cette question, car il ne dispose pas de toutes les informations. C'est pourquoi à peine $60 \%$ des repreneurs y ont répondu. Les résultats sont les suivants: pour $43 \%$ des $\mathrm{FBO}$, il n'y avait qu'un seul candidat à la succession faisant partie de la famille. Dans pareil cas, on peut parler de "dauphin». Seuls $4 \%$ des successeurs effectivement choisis dans le cadre de FBO indiquent avoir dû s'imposer face à des candidats extérieurs à la famille.

A l'inverse, 10 à $14 \%$ des successeurs extérieurs à la famille ont dû s'imposer face à des candidats issus de la famille. La plupart du temps, il n'y avait alors qu'un seul candidat issu de la famille. Au moins un quart des repreneurs par MBO ont dû s'imposer face à d'autres candidats internes à l'entreprise. En ce qui concerne les repreneurs par $\mathrm{MBI}, 16 \%$ se sont imposés face à des candidats internes à l'entreprise. Que ce soit pour les MBO ou les MBI, plus d'un quart des successeurs a par ailleurs dû s'imposer face à des candidats extérieurs.

Dans plus de la moitié des cas de $\mathrm{MBI}$, les candidats ont pris directement contact avec le prédécesseur. II est intéressant de noter que la médiation par le biais d'amis ou de connaissances joue un rôle plus important que celle via des intermédiaires professionnels. Les participants à l'enquête avaient aussi la possibilité d'indiquer d'autres canaux de contact que ceux proposés dans le questionnaire. Ainsi, le canal le plus souvent indiqué est l'annonce (dans le journal). Des fiduciaires, fournisseurs, liquidateurs, conseils de fondations et chasseurs de têtes ont également joué un rôle.

\section{Durée et organisation de la transmission}

La succession d'entreprise est un projet pour lequel différentes phases et étapes jalons doivent être planifiées et réparties sur plusieurs mois voire plusieurs années. Combien de temps s'écoule-t-il entre le premier contact entre le vendeur et l'acheteur et la reprise proprement dite de la direction de l'entreprise?

Comme le montre la figure 40, la durée entre le premier contact et le transfert effectif de la responsabilité varie sur un large spectre. Sans surprise, c'est pour les FBO que cette durée est la plus longue (6,5 ans) et pour les $\mathrm{MBI}$ qu'elle est la plus courte (1,6 an). Par expérience et d'après les recommandations pratiques, on sait qu'il faut veiller à ce que la période de transmission soit aussi courte que possible. Plus cette période est longue, plus il est probable que certaines hypothèses de base ou certains accords soient modifiés du fait des transformations de l'environnement, notamment dans le cas d'un MBI.
Figure 40

\section{Temps entre premier contact et transfert de responsabilité}

Part des entreprises par type de transmission, $n=368$

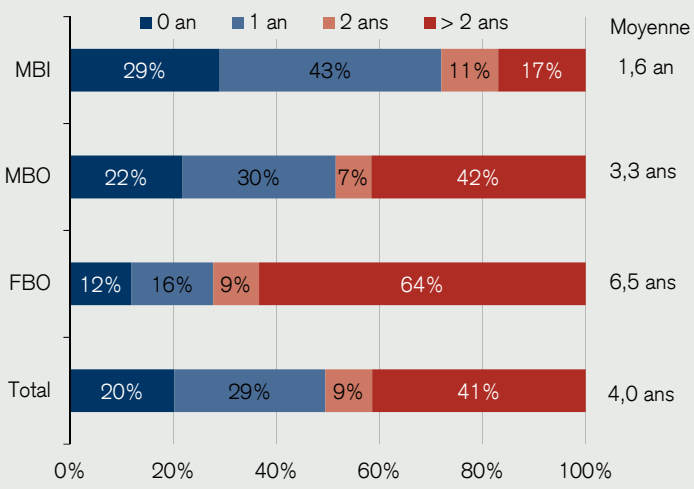

Source: Enquête PME Credit Suisse 2013
Figure 41

\section{Echelonnement des transferts de propriété et de} direction

Part des entreprises par type de transmission, $n=378$

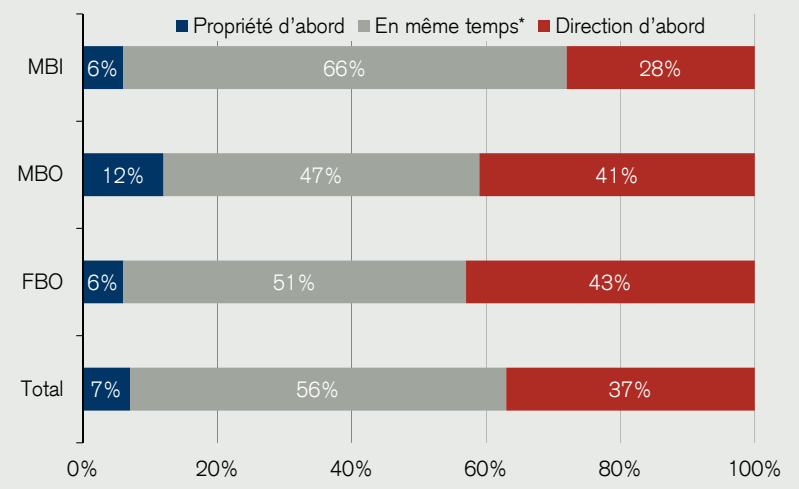

Source: Enquête PME Credit Suisse 2013; *durant la même année calendaire 
La direction et la propriété sont transmises séparément dans près de la moitié des cas

\section{La transmission de la propriété traîne en longueur}

La transmission de la direction se fait rapidement
Dans une succession d'entreprise, il convient de régler à la fois le transfert de la direction et celui de la propriété. Dans près de la moitié des cas, la propriété et la direction n'ont pas été transmises en même temps. Au lieu de cela, le vendeur a retenu dans la plupart des cas une certaine part de l'entreprise et a transmis la direction avant la propriété (figure 41). Comme on pouvait s'y attendre, c'est de loin dans le groupe des acheteurs externes que la transmission de la propriété et celle de la direction ont le plus souvent lieu simultanément. Dans plus d'un quart des cas, la direction a été reprise d'abord, ce qui indique généralement l'existence d'un prêt du vendeur (seller loan) ou de plans de financement similaires pour la reprise de l'entreprise. En cas de $\mathrm{MBO}$, on peut supposer que les successeurs potentiels internes à l'entreprise acquièrent relativement tôt des participations dans l'entreprise à hauteur d'un certain pourcentage, ce qui permet de les lier à l'entreprise et/ou de les faire participer au succès obtenu en commun et donc de favoriser le financement de la succession.

Des analyses approfondies montrent que si la propriété est transmise d'abord, alors la transmission de la direction intervient généralement un à deux ans plus tard. Dans le cas inverse, I'horizon temporel s'allonge. Dans plus d'un quart des cas où la direction a été transmise d'abord, le gérant n'a acquis la majorité des parts de l'entreprise qu'au bout de dix ans ou plus. Dans les cas les plus extrêmes, il s'est écoulé 40 ans entre la transmission de la direction et celle de la propriété. On peut supposer que dans ces cas, la propriété n'a été transférée au successeur qu'au moment de l'héritage (cas de FBO) ou sinon que le vendeur a gardé des participations dans I'entreprise jusqu'à sa mort à des fins de financement (cas de MBI). En ce qui concerne la durée du processus de transmission de la propriété, les résultats sont très variables. C'est lors des transmissions intrafamiliales qu'il s'écoule le plus de temps entre l'acquisition des premières parts et celle de la majorité de la propriété de l'entreprise. Cette période est nettement plus courte en cas de MBO et surtout en cas de MBI.

La durée du processus allant d'une reprise partielle à la reprise complète de la direction de l'entreprise est généralement assez courte. Dans la majorité des cas, l'entrée au sein de la direction et la reprise des responsabilités de gérance s'effectuent en l'espace de quelques mois (figure 42). On constate cependant de grandes différences suivant le type de repreneur: plus de $40 \%$ des successeurs familiaux coopèrent d'abord pendant plus de deux ans à la direction de l'entreprise avant d'endosser la responsabilité de la gérance. Pour les MBI, le transfert de la responsabilité de gérance est immédiat dans $90 \%$ des cas.

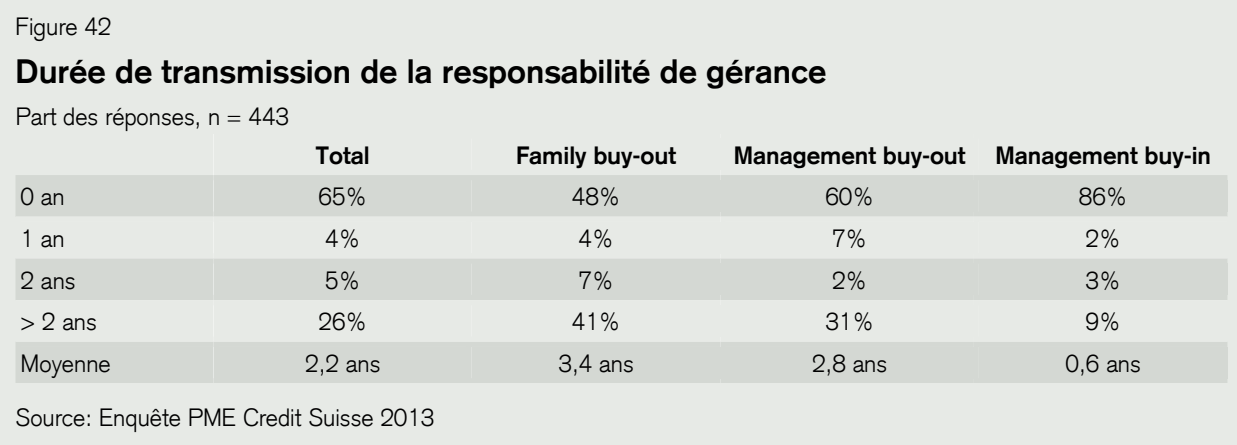

\section{Outils pour le processus de rapprochement}

Le processus de succession peut également être appréhendé comme un processus de rapprochement entre le vendeur et l'acheteur visant à ce que ces derniers fassent affaire ensemble sur une base de confiance mutuelle. Ce rapprochement est jalonné d'étapes de formalisation, allant de l'élaboration d'un profil d'exigences pour le successeur jusqu'au règlement du comportement après la transmission de l'entreprise, en passant par le processus de due diligence et la définition d'un plan de communication et de formation.

Nous avons d'abord examiné dans quelles sociétés il y avait un catalogue d'exigences destiné au successeur lors de la transmission. Dans près de la moitié des cas de reprise, il n'y en avait
Souvent, aucun profil d'exigences n'est élaboré 
pas (figure 43). Comme on pouvait $s^{\prime} y$ attendre, la formalisation est plus importante pour les $\mathrm{MBI}$ que pour les $\mathrm{MBO}$, et pour ces deux modes de transmission que pour les FBO. La faible prévalence d'un profil d'exigences doit être vue d'un œil critique. Compte tenu de la complexité et la rapidité croissantes de notre monde, les exigences envers un dirigeant augmentent sans cesse. Dans ce contexte, nous recommandons aux cédants de réfléchir activement à un possible profil d'exigences pour leur successeur. Surtout si l'entrepreneur souhaite que la société, l'œuvre de sa vie, continue de prospérer même après le passage de témoin.
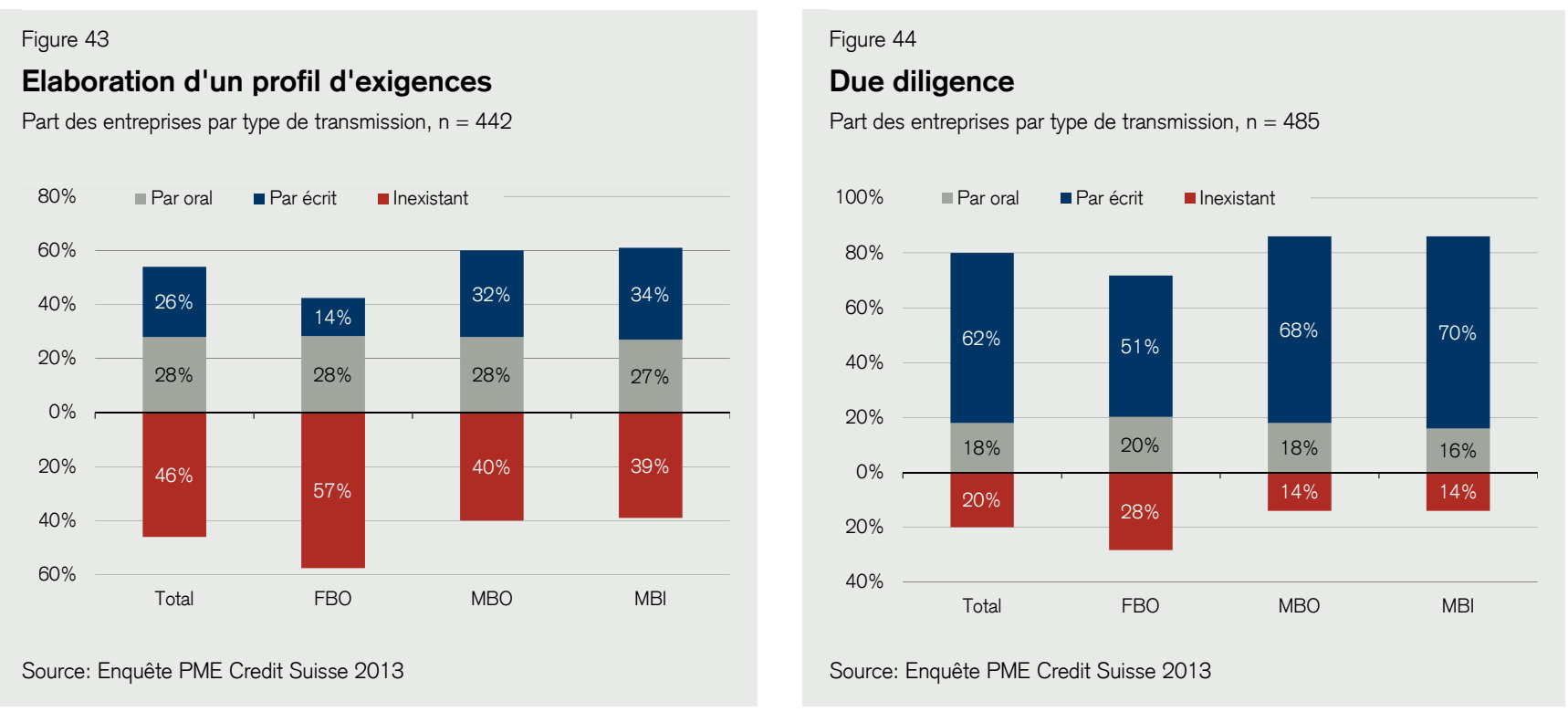

La due diligence s'est établie mais on y renonce encore souvent en cas de FBO

\section{Les plans de formation pour successeur plus fréquents en cas de FBO}

Dans le cadre du processus de négociation, il est d'usage de procéder à un contrôle dit de «due diligence», c'est-à-dire à un audit systématique et rigoureux de l'entreprise. Celui-ci est surtout effectué dans le cadre de MBO ou de MBI (figure 44). C'est dans les cas de MBI où les partenaires ne se connaissent pas encore en amont que l'asymétrie de l'information entre vendeur et repreneur est la plus grande..$^{25} \mathrm{La}$ due diligence est un instrument permettant de percer à jour les «entreprises boîtes noires». Le contrôle de due diligence est également recommandé pour les successeurs par FBO, même si en pratique il est peu utilisé dans ce cas. Même lorsqu'on connait l'entreprise depuis sa plus tendre enfance, un passage en revue systématique et détaillé de celle-ci révèle souvent de nouveaux éléments. De plus, les résultats objectifs et transparents du contrôle de due diligence permettent de mieux évaluer la situation actuelle et le potentiel de développement de l'entreprise. Le contrôle de due diligence a bien entendu un certain coût. Mais comme l'indique clairement sa diffusion plutôt vaste, le bénéfice d'un tel contrôle dépasse typiquement son coût.

Une fois que les parties se sont mises d'accord sur les grandes lignes, elles peuvent passer dans la pratique aux phases d'initiation. II est frappant de constater que les plans de formation pour le successeur ne sont pas vraiment monnaie courante sur l'ensemble de l'échantillon (figure 45). C'est parmi les FBO que la formation systématique du successeur est la plus répandue, et parmi les MBO qu'elle l'est le moins. Ceci s'explique par le fait que dans un MBO, les successeurs travaillent généralement déjà depuis plusieurs années au sein de l'entreprise. Mais même en cas de $\mathrm{MBO}$, il est recommandé d'élaborer un plan de formation spécifique aux tâches. Si par exemple l'ancien responsable de la production est choisi comme successeur, il est possible que celui-ci manque d'expérience et de connaissances en vente. De telles lacunes peuvent être identifiées facilement en amont de la transmission et comblées à l'aide d'une formation. Comme le montre notre étude, les successeurs familiaux sont souvent déjà connus plusieurs années avant la transmission d'entreprise. Dans de tels cas, il est possible d'intégrer très 
tôt dans l'entreprise le successeur potentiel et de l'affecter ainsi aux domaines de responsabilité les plus variés.

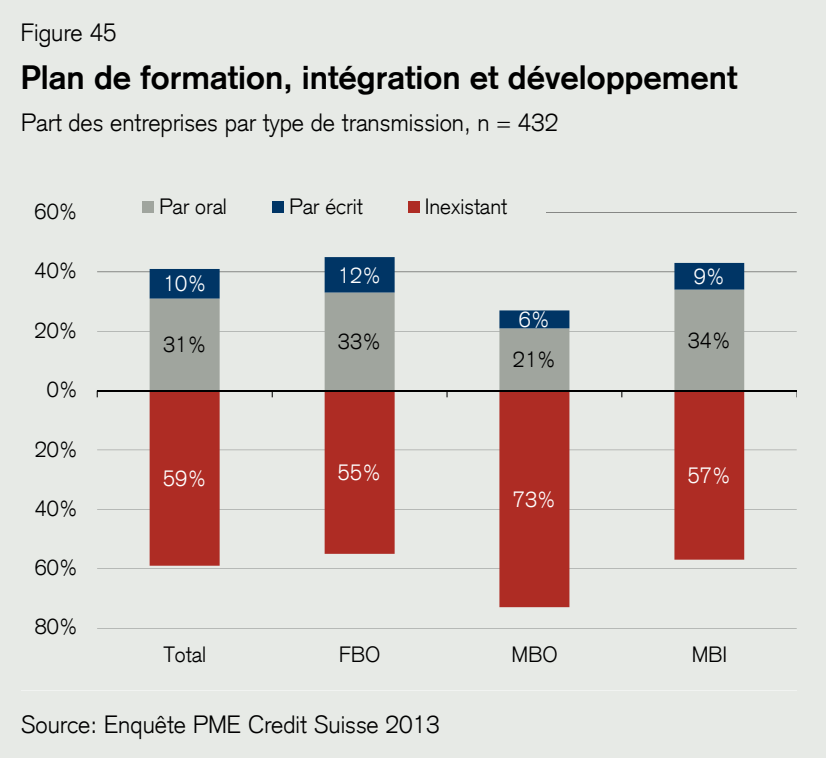

Part des entreprises par type de transmission, $n=432$

\section{Figure 46}

\section{Plan de communication}

Part des entreprises par type de transmission, $n=438$

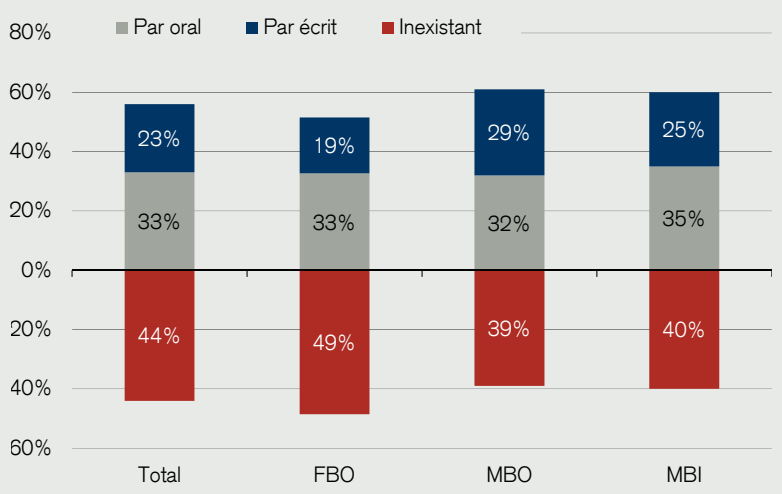

Source: Enquête PME Credit Suisse 2013
Pas de plan de communication dans $44 \%$ des cas

Plus de $\mathbf{5 0} \%$ des entreprises règlent le comportement des prédécesseurs

Conscience de l'importance de la phase de "postsuccession"

Les entrepreneurs familiaux restent plus longtemps...
Lorsqu'approche la réalisation de la succession d'entreprise, il convient de définir un plan de communication indiquant qui peut dire quoi à qui et à quel moment et précisant les contenus concrets de la communication ainsi que les sujets à ne pas aborder. Ces plans de communication sont inexistants dans $44 \%$ des entreprises et ils ne sont consignés par écrit que dans $23 \%$ des entreprises (figure 46). Les plans de communication aident à éviter les malentendus et les incertitudes pouvant en résulter à l'intérieur et à l'extérieur de l'entreprise. Notre étude montre que les entrepreneurs s'inquiètent en général souvent des successions de leur entreprise dans leur environnement commercial. Les bruits non confirmés et les informations erronées finissent souvent par inquiéter les partenaires commerciaux. Ceci pourrait être évité grâce à des plans de communication.

Puis se pose enfin la question de savoir si le comportement du prédécesseur après la reprise doit ou non être réglé durant la phase de transmission. D'après notre enquête, le comportement «post-succession» du prédécesseur a été réglé dans plus de la moitié des $\mathrm{MBO}$ et $\mathrm{MBI}$ (figure 47). Entre $\mathrm{MBO}$ et $\mathrm{MBI}$, il n'y a pratiquement pas de différence, tandis que le comportement du prédécesseur après la transmission est beaucoup moins souvent fixé dans les cas de FBO. Nous l'expliquons par le fait que le processus de transmission dure plus longtemps en cas de FBO et qu'il est par ailleurs incomparablement plus compliqué pour un enfant d'exiger des changements de comportement de la part de ses parents. En particulier en cas de FBO, il est cependant recommandé de fixer préventivement par écrit la répartition des rôles. Si on ne consigne pas par écrit le comportement post-succession souhaité, chacune des parties aura ses propres attentes. Et si ces attentes divergent, cela provoquera des tensions et des conflits. En particulier en cas de FBO, ceci peut avoir de lourdes répercussions, car les différents membres de la famille doivent également continuer de se côtoyer en privé et ne peuvent tout simplement pas s'éviter. L'absence d'une convention de ce type peut donc conduire à ce que d'éventuels conflits se transmettent de l'entreprise à la famille.

Comme nous avons pu le montrer, le règlement de la phase post-succession joue un rôle. II est intéressant de se demander à présent comment celui-ci est effectivement mis en place dans la pratique. Ainsi, nous avons avant tout cherché à savoir si le prédécesseur disposait encore d'un bureau après le règlement formel, combien d'heures de travail il passait encore dans l'entreprise et $s^{\prime}$ il exerçait toujours une influence sur les affaires du point de vue du repreneur. Enfin, nous avons également sondé la satisfaction des participants concernant l'ensemble du processus.

Le bureau d'un entrepreneur est souvent le centre de ses intérêts pendant des décennies au point de devenir une partie de son identité. On peut donc s'attendre à ce que les ex- 
entrepreneurs ne quittent pas immédiatement leur bureau après la transmission. Les résultats de notre étude montrent que les prédécesseurs avaient encore un bureau pendant 4,2 ans en moyenne après la transmission de l'entreprise (figure 48). C'est surtout en cas de FBO que le prédécesseur restait longtemps présent avec un bureau. En cas de $\mathrm{MBI}$, la génération sortante quitte la plupart du temps son bureau dans un délai d'un an. II n'y a pas de règle générale concernant une durée optimale. Les parties doivent toutefois avoir conscience des avantages et inconvénients du maintien en activité du prédécesseur au sein de l'entreprise. Ils doivent notamment évaluer si le bénéfice que peut tirer le successeur de l'expérience du prédécesseur est supérieur aux coûts engendrés par une prise d'influence indésirable et contre-productive. Par conséquent, les aspects tels que le bureau, le temps de présence, l'entrée au conseil d'administration etc. doivent être réglés.

\section{Figure 47}

\section{Règlement du comportement «post-succession"}

Part des entreprises par type de transmission, $n=447$

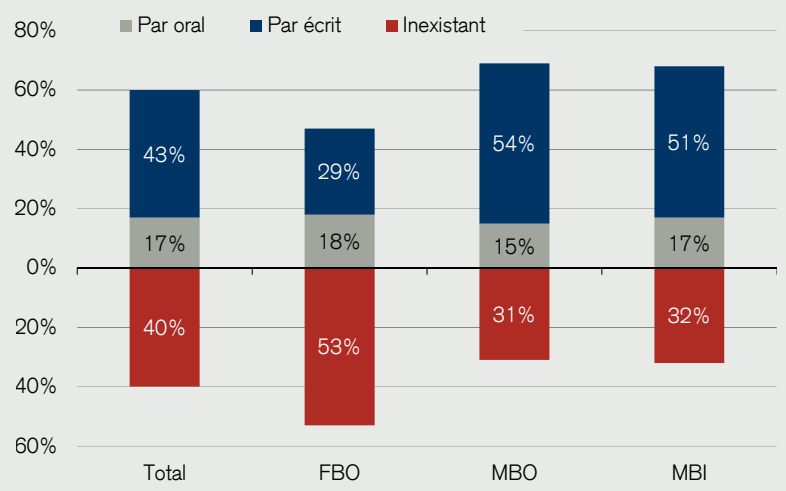

Source: Enquête PME Credit Suisse 2013

\section{Figure 48}

\section{Jusqu'à quand le prédécesseur avait-il encore un} bureau fixe?

Part des entreprises par type de transmission, $n=369$

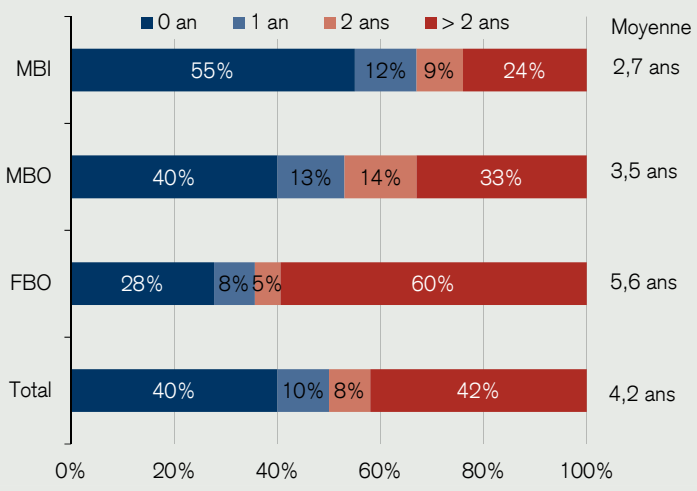

Source: Enquête PME Credit Suisse 2013;

\section{... en moyenne avec un taux d'occupation de $50 \%$}

\section{Prise d'influence du prédécesseur surtout en cas de FBO}

Ci-après, nous mesurons l'intensité de la collaboration du prédécesseur dans l'entreprise en nous basant sur son temps de travail hebdomadaire moyen. Là encore, on constate que la transmission ne signifie pas son départ immédiat à la retraite. Plus de la moitié des exentrepreneurs fréquente encore le bureau de l'entreprise plusieurs heures par semaine. On note une différence particulièrement importante entre les transmissions au sein de la famille (durée hebdomadaire moyenne du travail supérieure à 20 heures après la transmission) et les transmissions à l'extérieur de la famille (durée hebdomadaire moyenne du travail inférieure à 10 heures après la transmission).

Parallèlement, le successeur doit prendre ses marques dans l'entreprise et développer son autonomie entrepreneuriale. II est donc intéressant de se demander dans quelle mesure le successeur s'est senti influencé par son prédécesseur dans ses activités entrepreneuriales, notamment lors de la mise en œuvre de nouvelles modifications. Les résultats montrent qu'environ $80 \%$ des repreneurs se sont sentis peu voire pas du tout influencés, en particulier après des reprises par MBO ou MBI (figure 49). Le bilan est différent pour les successeurs familiaux: plus d'un tiers d'entre eux ont indiqué que leur prédécesseur avait tenté de les influencer moyennement voire (très) fortement. 


\section{Figure 49}

\section{Influence lors de l'introduction de modifications}

Part des entreprises par type de transmission, $n=504$

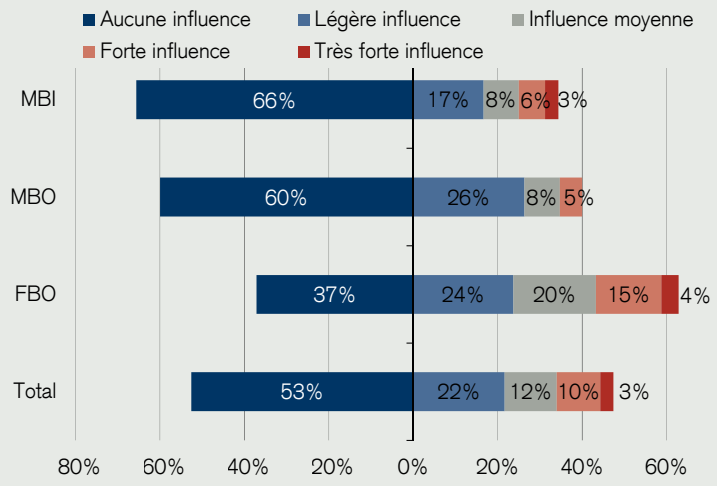

Source: Enquête PME Credit Suisse 2013
Figure 50

\section{Nature de la prise d'influence}

Part des entreprises par type de transmission ${ }^{*}, n=445$

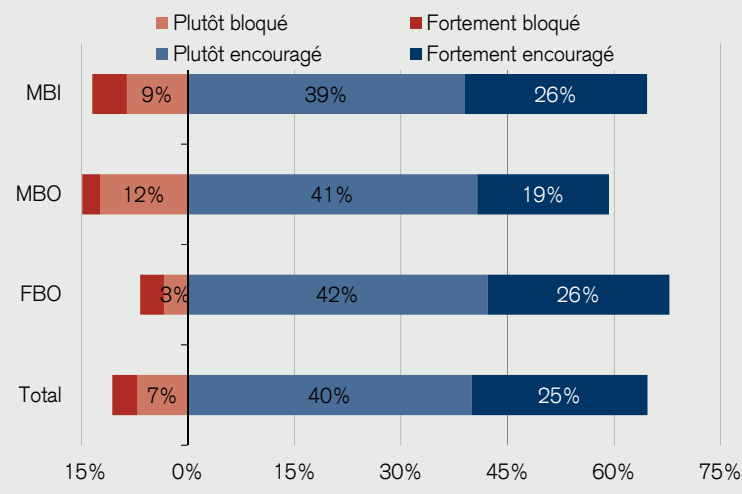

Source: Enquête PME Credit Suisse 2013; *la réponse «Parfois bloqué, parfois encouragé» n'est pas représentée
Une prise d'influence des prédécesseurs majoritairement positive
La détermination du prix et le financement doivent être traités séparément

$20 \%$ reprennent gratuitement l'entreprise familiale, les collaborateurs bénéficient d'un léger rabais
Les repreneurs amis extérieurs à l'entreprise bénéficient d'un plus gros rabais que les collaborateurs
Dans un second temps, nous avons demandé aux repreneurs si leur prédécesseur avait plutôt bloqué ou encouragé leurs tentatives d'introduction de modifications. Dans l'ensemble, on constate que les repreneurs se sont sentis encouragés positivement (figure 50). Les chiffres relatifs aux obstructions sont surprenants: ces comportements ont été ressentis beaucoup plus souvent par les successeurs en MBO ou MBI que par les successeurs familiaux. Ce résultat est particulièrement intéressant si on le met en rapport avec les résultats ci-dessus selon lesquels I'influence «post-succession» est la plus élevée dans les cas de transmission familiale On pourrait en conclure que lors d'un $\mathrm{MBO}$ ou $\mathrm{MBI}$, il n'est pas souhaitable que l'ancien CEO garde une influence, ne serait-ce que pour la satisfaction du repreneur.

\section{Détermination du prix et financement}

Lors d'une transmission d'entreprise, il convient de déterminer d'abord le prix, puis dans un second temps d'assurer le financement. On gagnera à séparer mentalement ces deux étapes, même si dans la pratique elles sont souvent associées. Pour l'évaluation de l'entreprise, on peut utiliser différentes méthodes, qui aboutissent généralement à des écarts relativement importants.

Dans la présente étude, nous ne nous sommes pas intéressés aux méthodes d'évaluation mais avons considéré que le repreneur connaissait la valeur de marché de l'entreprise. Nous avons demandé aux participants à quel prix ils avaient acquis l'entreprise en pourcentage de la valeur de marché de celle-ci. 20\% des repreneurs en FBO ont obtenu l'entreprise "gratuitement». Un tiers des repreneurs en $\mathrm{MBI}$ a dû débourser la totalité de la valeur de marché, contre seulement un quart des entrepreneurs en FBO. Beaucoup de successeurs familiaux ont bénéficié d'un rabais généreux compris entre 20 et $60 \%$ du prix du marché. Ce rabais appelé "family discount» s'élève en moyenne à $42 \%$ de la valeur de marché. En revanche, les repreneurs en MBO bénéficient généralement d'un léger rabais inférieur à 20\%.

Parmi les $\mathrm{MBI}$, nous avons opéré ci-après une distinction supplémentaire. La figure 51 montre que lors d'une transmission d'entreprise à un ami dans le cadre d'un $\mathrm{MBI}$, le rabais consenti est plus élevé que pour un $\mathrm{MBO}$, un $\mathrm{MBI}$ à une personne inconnue auparavant ou même un $\mathrm{MBI}$ à un partenaire commercial actuel (p. ex. fournisseur, client, concurrent). Nous pouvons en outre supposer que dans notre échantillon - et comme on le constate également dans la pratique - il n'y avait pratiquement pas d'entreprises reprises par des investisseurs financiers sous forme de sociétés de private equity. 
Figure 51

\section{Rabais par rapport à la valeur de marché}

Part moyenne de la valeur de marché par type de transmission, $n=455$

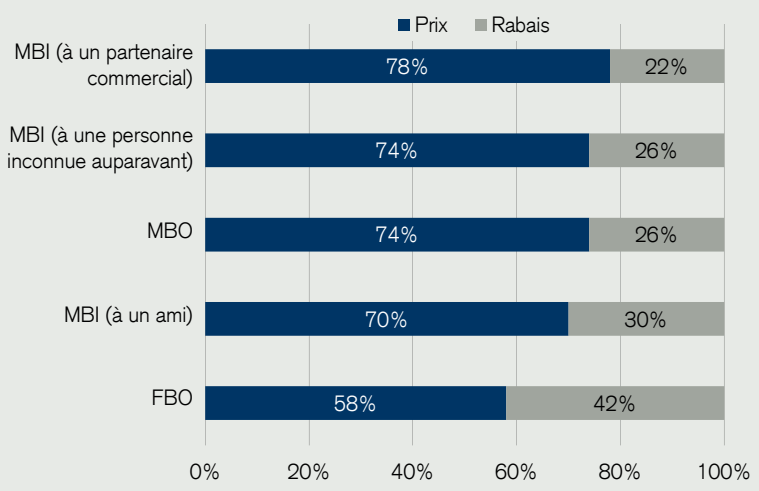

Source: Enquête PME Credit Suisse 2013
Figure 52

\section{Structure de financement de la succession}

Part moyenne de chaque forme de financement par type de transmission

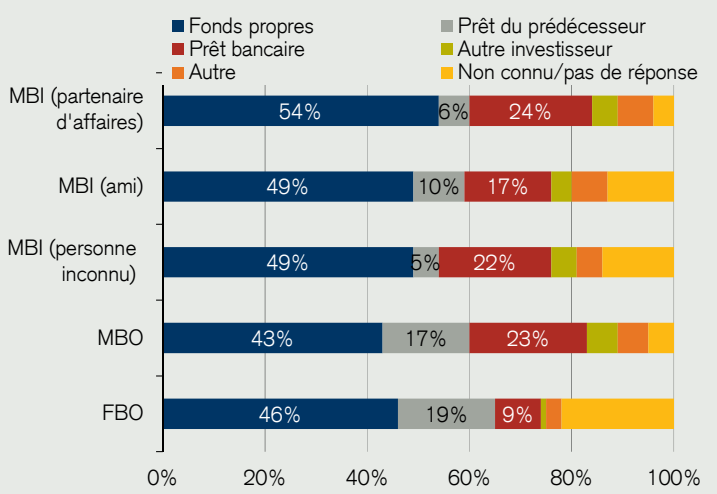

Source: Enquête PME Credit Suisse 2013

\section{La succession nécessite une base solide de fonds propres}

Les prêts de vendeurs sont une affaire de confiance

Financement bancaire principalement pour les MBO et MBI
Dès que le prix a été négocié et fixé, se pose la question du financement. Divers instruments de financement par fonds propres ou étrangers sont alors disponibles et peuvent même être coordonnés de manières très différentes suivant la forme de transmission choisie. Dans tous les cas, les entrepreneurs doivent assurer une base solide sous forme de fonds propres, qui représentent dans la pratique entre $43 \%$ et $54 \%$ (figure 52). En cas de $\mathrm{FBO}$, une partie du financement par fonds propres est généralement apportée sous forme d'avancement d'hoirie ou de donation, pour autant que les considérations relatives à l'équité de traitement dans la famille le permettent.

Le prêt du prédécesseur est surtout utilisé en cas de FBO (19\%) et de MBO (17\%), ainsi qu'en cas de $\mathrm{MBI}$ à un ami (10\%). II apparaît clairement que le recours à cette solution est avant tout une question de confiance. Plus la distance personnelle entre le successeur et le prédécesseur est importante, moins il est probable que le vendeur participe personnellement au financement de la reprise de l'entreprise.

Avec une part comprise entre $17 \%$ et $24 \%$, le financement bancaire classique est une composante essentielle du financement de reprise de sociétés par MBO et MBI. Pour y avoir accès, le repreneur doit avant tout établir clairement la viabilité et le potentiel de l'entreprise ainsi que sa capacité à l'exploiter. Ceci repose non seulement sur les chiffres passés, mais également et surtout sur un business plan crédible.

\section{Processus de transmission: enseignements et recommandations pour la pratique}

- Assurez-vous de pouvoir discuter du thème de la succession d'entreprise avec des tiers. Ce peut être le conseil d'administration, un conseiller, un ami, une connaissance ou votre banque maison.

- La transmission de l'entreprise peut se faire en une seule fois ou de manière échelonnée, la propriété et la direction peuvent être transmises simultanément ou de manière décalée. Les possibilités sont nombreuses. L'important est que les engagements soient toujours garantis.

- Plus le processus de transmission est long, plus il est important (en particulier en cas de FBO) de formaliser les conditions-cadres et les processus afin d'éviter les conflits.

- La valeur de l'entreprise n'est pas son prix. Le vendeur doit savoir quel est le prix minimal acceptable. Quel rabais est-il prêt à consentir pour que l'entreprise se poursuive?

- Une fois le prix convenu, il faut le financer. Déterminez la quantité de fonds propres requis et réfléchissez à la manière de les compléter par des prêts et fonds de tiers. 


\section{Evaluation de la performance de l'entreprise}

Les repreneurs extérieurs considèrent souvent l'entreprise reprise comme peu performante
Reste à s'intéresser à la manière dont est évaluée la performance des entreprises transmises. Voyons d'abord comment les repreneurs évaluent leur entreprise par rapport à la concurrence au moment de la reprise. La figure 53 montre qu'en moyenne, près de $30 \%$ des repreneurs considèrent leur entreprise comme peu performante (10\% «bien plus mauvaise», $21 \%$ "un peu plus mauvaise») par rapport à la concurrence au moment de la reprise. La distinction entre FBO, $\mathrm{MBO}$ et $\mathrm{MBI}$ montre que ce sont surtout les candidats à un $\mathrm{MBI}$ qui considèrent l'entreprise reprise comme beaucoup moins performante. Nous partons donc du principe que les candidats à un $\mathrm{MBI}$ évaluent l'objet repris d'un œil plus (auto)critique que les candidats à un FBO ou MBO.
Figure 53

\section{Performance face aux concurrents lors de la reprise \\ Part des réponses (réponse «comparable» exclue), $n=508$}

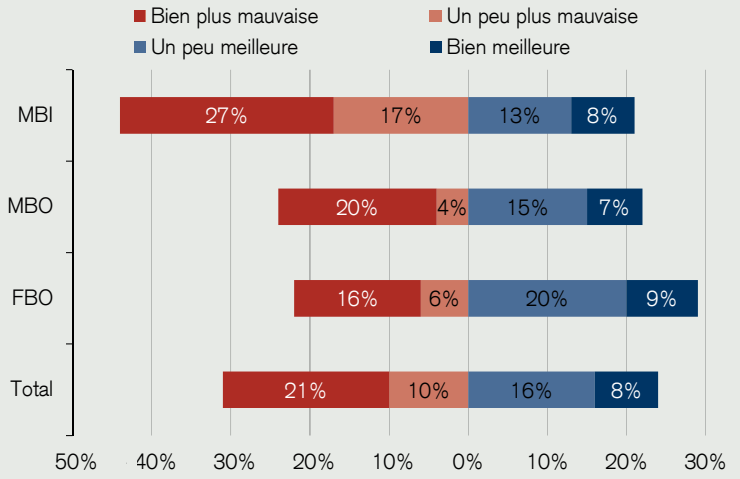

Source: Enquête PME Credit Suisse 2013

\section{Figure 54}

\section{Performance actuelle vs performance lors de la reprise}

Part des réponses (réponse «comparable» exclue), $\mathrm{n}=514$

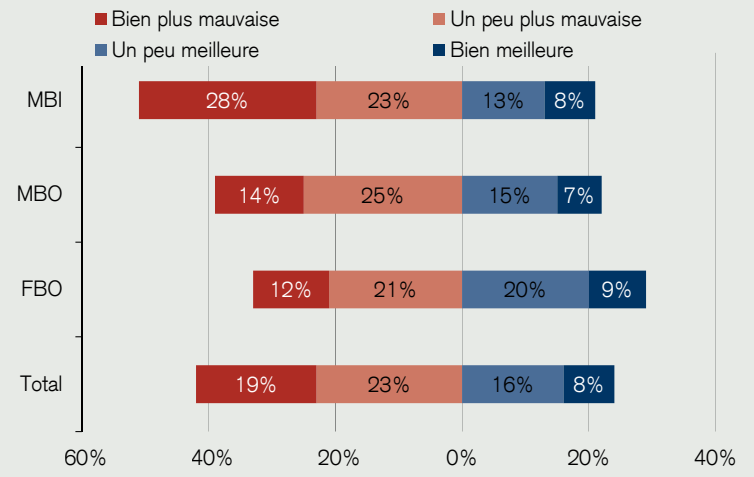

Source: Enquête PME Credit Suisse 2013
Les repreneurs familiaux regardent positivement l'entreprise reprise

Les évaluations s'harmonisent après la reprise
A l'inverse, l'image qu'ont de leur entreprise les repreneurs familiaux est plutôt teintée d'optimisme. 29\% des successeurs considèrent l'entreprise comme «un peu meilleure» (20\%) ou «bien meilleure» (9\%) que ses concurrents. Ceci peut s'expliquer de la manière suivante: comme les successeurs familiaux acquièrent généralement l'entreprise à un prix inférieur à sa valeur de marché (cf. chapitre "Détermination du prix et financement»), ils ne remettent pas en question ses résultats avec la même rigueur. De plus, leur attachement affectif à l'entreprise depuis de longues années peut altérer leur objectivité.

En examinant l'évaluation de la performance actuelle de l'entreprise par rapport à sa performance au moment de la reprise, on remarque que les entrepreneurs en $\mathrm{MBI}$ estiment que l'entreprise va beaucoup mieux aujourd'hui ( $28 \%$ des cas) qu'au moment de la reprise (figure 54). Avec $12 \%$ seulement, c'est chez les repreneurs en FBO que cette part est la plus faible. Nous pouvons donc en déduire qu'un prix d'achat relativement bas et une faible part de financement sur fonds étrangers n'ont pas forcément un effet positif sur la performance de I'entreprise. On pourrait même formuler l'hypothèse selon laquelle lorsque le propriétaire prend davantage de risques, cela accentue la pression sur le développement et la performance.

\section{Performance de l'entreprise: enseignements et recommandations pour la pratique}

- La santé de l'entreprise avant la succession détermine en grande partie son aptitude à faire l'objet d'une reprise. Les repreneurs extérieurs en particulier souhaitent exploiter un potentiel d'avenir et non pas uniquement acquérir de la substance.

- Des chiffres sains concernant le passé constituent une bonne base de départ.

- Un business plan est nécessaire pour le futur.

- Plus vite les successeurs peuvent investir dans le futur, meilleur c'est. 


\section{Informations complémentaires}

www.credit-suisse.com/succession

- www.cfb.unisg.ch/wb

nww.kmunext.ch

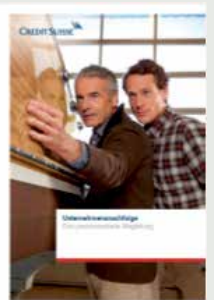

Succession d'entreprise

Un guide pratique

Credit Suisse AG

www.credit-suisse.com/entreprises 


\section{Notification}

\section{Certification par les analystes}

Chacun des analystes mentionnés dans le présent document certifie que les points de vue exprimés dans cette brochure à l'endroit des entreprises et des titres qu'il ou elle est amené(e) à évaluer reflètent précisément son opinion personnelle. II affirme que sa rémunération n'est et ne sera jamais liée, directement ou indirectement, à des recommandations et opinions particulières émises dans ce document.

Les analystes Knowledge Process Outsourcing (KPO) mentionnés dans le présent rapport sont employés par Credit Suisse Business Analytics (India) Private Limited.

\section{Indications importantes}

Le Credit Suisse publie ses brochures au moment qu'il estime le plus approprié. C'est le cas dès lors qu'il juge que l'évolution de l'entreprise, du secteur ou du marché soumis à son évaluation est susceptible d'avoir des répercussions sensibles sur les points de vues et opinions exprimés dans la brochure. Le Credit Suisse se fait fort de publier uniquement des analyses impartiales, indépendantes, honnêtes, claires et intelligibles.

Le code de conduite du Credit Suisse, auquel la totalité des employés doit adhérer, est accessible par Internet sous:

https://www.credit-suisse.com/governance/doc/code_of_conduct_fr.pdf

Pour des détails complémentaires sur la méthodologie de notation du Credit Suisse, veuillez vous référer aux informations sur l'indépendance des recherches financières qui se trouvent sous:

https://www.credit-suisse.com/legal/pb_research/independence_en.pdf

L'analyste responsable de la rédaction de cette brochure se voit rétribué en fonction de plusieurs paramètres parmi lesquels la totalité des revenus touchés par le Credit Suisse, dont une partie est générée par les activités d'investment banking du Credit Suisse.

\section{Notifications complémentaires pour les juridictions suivantes}

Royaume-Uni: En ce qui concerne la notification des informations sur titres à revenu fixe pour les clients de Credit Suisse (UK) Limited et de Credit Suisse Securities (Europe) Limited, veuillez téléphoner au +4144333 3399.

Pour toutes informations complémentaires y compris les notifications relatives à tout autre émetteur, veuillez vous référer au site de Credit Suisse Global Research Disclosure sous:

https://www.credit-suisse.com/disclosure

\section{Clause de non-responsabilité générale / Information importante}

Pour une discussion sur les risques afférents aux placements dans les titres mentionnés dans ce rapport, veuillez consulter ce lien Internet:

https://research.credit-suisse.com/riskdisclosure

Les références effectuées dans cette brochure au Credit Suisse comprennent ses succursales et ses sociétés affiliées. Pour plus d'informations sur notre structure, veuillez consulter le lien suivant:

https://www.credit-suisse.com/who_we_are/fr/

Les informations et opinions exprimées dans ce rapport sont celles du département Global Research de la Division Private Banking du Credit Suisse au moment de la rédaction; toute modification demeure réservée sans préavis. Les opinions exprimées en relation avec un titre spécifique dans ce rapport peuvent être différentes ou ne pas concorder avec les observations et les opinions du département Research de la Division Investment Banking du Credit Suisse en raison de différences dans les critères d'évaluation. Ce rapport n'est pas destiné à être distribué à, ou utilisé par, quelque personne ou entité que ce soit qui serait citoyenne, résidente ou située dans une localité, un État, un pays ou une autre juridiction où une telle distribution, publication, disponibilité ou utilisation serait contraire à la législation ou réglementation ou soumettrait Credit Suisse AG, la banque suisse ou ses succursales et sociétés affiliées (le "CS») à des obligations d'enregistrement ou de licence au sein de ladite juridiction. Sauf indication contraire, tous les éléments de ce rapport sont la propriété du CS et soumis à droits d'auteur. Aucun élément, ou son contenu, ni aucune copie de ce dernier, ne peut être altéré, transmis, copié ou distribué à toute autre partie de quelque manière que ce soit, sans l'accord écrit explicite préalable du CS. Toutes les marques de commerce, marques de service et logos utilisés dans ce rapport sont des marques de commerce, des marques de service ou des marques de commerce ou des marques de service enregistrées du CS ou de ses sociétés affiliées.

Les informations, outils et éléments présentés dans ce rapport sont fournis uniquement à titre d'information et ne doivent pas être utilisés ou considérés comme une offre ou une invitation à acheter, vendre ou souscrire à des titres ou autres instruments financiers. Le CS n'offre pas de conseils sur les conséquences d'ordre fiscal liées aux investissements et vous recommande de consulter un conseiller fiscal indépendant. Veuillez tout particulièrement noter que les bases et niveaux d'assujettissement à l'impôt peuvent varier.

Le CS est d'avis que les informations et les opinions publiées dans l'appendice sous «Notification» sont exactes et complètes. Les informations et les opinions figurant dans les autres sections du rapport ont été obtenues ou tirées de sources jugées fiables par le CS; toutefois le CS décline toute responsabilité quand à leur exactitude ou leur intégralité. Des informations complémentaires sont disponibles sur demande. Le CS décline toute responsabilité à l'égard de toute perte découlant de l'utilisation des éléments présentés dans ce rapport; cette exclusion de responsabilité ne saurait être invoquée dans les cas prévus par la législation ou la réglementation applicable au CS. Ce rapport ne doit pas être substitué à l'exercice d'un jugement indépendant comme fondement à la prise de décision. Le CS peut avoir émis ou pourrait émettre dans l'avenir une idée d'investissement concernant ce titre. Les idées d'investissement sont des opportunités à court terme basées sur des événements et des éléments déclencheurs sur les marchés, tandis que les notations des sociétés s'appuient sur la performance absolue escomptée sur une période de 12 mois tel que cela est défini dans la section notification. Les idées d'investissement et les notations de sociétés reflètent des hypothèses sous-jacentes et des méthodes d'analyse différentes. Par conséquent, il est possible que les idées d'investissement n'aillent pas dans le même sens que les notations de sociétés. En outre, le CS peut avoir publié d'autres rapports, ou pourrait en publier dans l'avenir, qui contredisent les informations présentées dans ce rapport ou qui en tirent des conclusions différentes. Ces rapports reflètent les diverses suppositions, visions et méthodes d'analyses des analystes qui les ont rédigés et le CS n'est nullement tenu de garantir que lesdits rapports soient portés à l'attention de tout destinataire du présent rapport. Le CS est impliqué dans plusieurs opérations commerciales en relation avec les entreprises mentionnées dans ce rapport. Ces opérations incluent notamment le négoce spécialisé, l'arbitrage des risques, les activités de tenue de marché et autres activités de négoce pour compte propre.

Les informations, opinions et évaluations présentées dans ce rapport reflètent le jugement émis par le CS à la date de publication initiale et sont susceptibles d'être modifiées sans préavis. Ce rapport peut fournir des adresses de sites web ou contenir des liens qui conduisent à ces sites. Sauf dans la mesure où le rapport fait état du contenu web du CS, le CS n'a pas procédé au contrôle des sites web liés et décline toute responsabilité quant au contenu desdits sites. Ces adresses ou hyperliens (y compris les adresses ou hyperliens vers le contenu web du site du CS) ne sont fournis que pour votre confort et votre information et le contenu des sites liés ne fait partie d'aucune manière du présent document. L'accès à un tel site web ou le suivi d'un tel lien par le biais de ce rapport ou via le site web du CS se fait à vos propres risques.

\section{Distribution des rapports de recherche}

A l'exception d'une éventuelle mention contraire, ce rapport est distribué par Credit Suisse AG, une banque suisse agréée et réglementée par l'Autorité fédérale de surveillance des marchés financiers. Allemagne: Credit Suisse (Deutschland) AG est agréée et réglementée par le Bundesanstalt für Finanzdienstleistungsaufsicht (BaFin); elle adresse à ses clients des études qui ont été préparées par l'une de ses sociétés affiliées. Australie: Ce rapport est distribué en Australie par Credit Suisse AG, Sydney Branch (CSSB) (ABN 17061700 712 AFSL 226896) seulement aux clients «Wholesale» comme défini à la section s761G of the Corporations Act 2001. CSSB ne garantit pas la performance des produits financiers mentionnés dans le rapport et ne fournit aucune assurance quant à la performance de ces produits. Bahamas: Ce rapport a été préparé par Credit Suisse AG, la banque suisse, et il est distribué pour le compte de Credit Suisse AG, Nassau Branch, société affiliée de la banque suisse, enregistrée en tant que broker-courtier auprès de la Securities Commission of the Bahamas. Bahreïn: Ce rapport est distribué par Credit Suisse AG, Bahrain Branch, qui est autorisée et réglementée par la Central Bank of Bahrain (CBB) comme un Investment Firm Category 2. Brésil: Les informations contenues dans les présentes ne constituent pas une offre publique de titres au Brésil et les titres mentionnés dans les présentes peuvent ne pas être enregistrés auprès de la Com- 
mission des valeurs mobilières du Brésil (CVM). Dubaï: Cette information est distribuée par Credit Suisse AG, Dubai Branch, dûment agréée et réglementée par la Dubai Financial Services Authority (DFSA). Les produits ou les services financiers liés ne sont disponibles qu'à des clients grossistes disposant d'actifs liquides de plus de 1 million d'USD qui disposent d'une expérience et d'une compréhension suffisantes pour participer aux marchés financiers dans une juridiction de grossiste et qui satisfont au critère de régulation afin de devenir client. Espagne: Ce rapport est distribué en Espagne par Credit Suisse AG, Sucursal en España, autorisée sous le numéro 1460 dans le registre de la Banco de España. France: Ce rapport est distribué par le Credit Suisse (France) qui est autorisé par le Comité des Etablissements de Crédit et des Entreprises d'Investissements (CECEI) en tant que fournisseur de services de placement. Credit Suisse (France) est placé sous la supervision et la réglementation de l'Autorité de Contrôle Prudentiel ainsi que de l'Autorité des Marchés Financiers. Gibraltar: Ce rapport est distribué par Credit Suisse (Gibraltar) Limited. Credit Suisse (Gibraltar) Limited est une entité légalement indépendante détenue en totalité par Credit Suisse et elle est réglementée par la Gibraltar Financial Services Commission. Guernesey: Le présent rapport est distribué par Credit Suisse (Guernsey) Limited, une entité juridique indépendante enregistrée à Guernesey sous le numéro 15197 et ayant son adresse enregistrée à Helvetia Court, Les Echelons, South Esplanade, St Peter Port, Guernesey. Credit Suisse (Guernsey) Limited est détenu à 100\% par le Credit Suisse et est régulé par la Guernsey Financial Services Commission. Inde: Ce rapport est distribué par Credit Suisse Securities (India) Private Limited ("Credit Suisse India»), qui est réglementé par le Securities and Exchange Board of India (SEBI) sous les numéros d'enregistrement SEBI INB230970637, INF230970637, INB010970631, INF010970631, et dont l'adresse enregistrée est 9th Floor, Ceejay House, Plot F, Shivsagar Estate, Dr. Annie Besant Road, Worli, Mumbai 400 018, Inde, tél. +91-22 6777 3777. Italie: Ce rapport est distribué en Italie par Credit Suisse (Italy) S.p.A., banque de droit italien inscrite au registre des banques et soumise à la supervision et au contrôle de la Banca d'Italia, de la CONSOB et est aussi distribué par Credit Suisse AG agissant à titre de banque suisse autorisée à fournir des prestations bancaires et financières en Italie. Japon: Ce rapport est publié uniquement au Japon par Credit Suisse Securities (Japan) Limited, Financial Instruments Dealer, Director-General of Kanto Local Finance Bureau (Kinsho) No. 66, membre de la Japan Securities Dealers Association, de la Financial Futures Association of Japan, de la Japan Investment Advisers Association et de la Type II Financial Instruments Firms Association. Credit Suisse Securities (Japan) Limited ne publiera ni ne transmettra ce rapport en dehors du Japon. Jersey: Ce rapport est distribué par Credit Suisse (Guernsey) Limited, succursale de Jersey, qui est réglementé par la Jersey Financial Services Commission. L'adresse professionnelle à Jersey de Credit Suisse (Guernsey) Limited, succursale de Jersey, est TradeWind House, 22 Esplanade, St Helier, Jersey JE2 30A. Luxembourg: Ce rapport est distribué par Credit Suisse (Luxembourg) S.A., une banque du Luxembourg autorisée et réglementée par la Commission de Surveillance du Secteur Financier (CSSF). Mexique: Les informations figurant dans la présente ne constituent pas une offre publique de titres au sens de la loi mexicaine sur les titres. Cette brochure ne sera pas publiée dans une quelconque publication ou moyen de communication à grand tirage au Mexique. Cette brochure ne contient aucune publicité concernant l'intermédiation ou l'offre de services bancaires ou de conseils en placement au Mexique ou à des citoyens mexicains. Qatar: Cette information a été distribuée par Credit Suisse Financial Services (Qatar) L.L.C qui a été autorisée et qui est réglementée par la Financial Centre Regulatory Authority (OFCRA) sous le $n^{\circ}$ OFC 00005 . Tous les produits et les services financiers liés ne sont disponibles qu'aux clients commerciaux ou aux contreparties du marché (tels que définis par la Qatar Financial Centre Regulatory Authority (OFCRA)), y compris les individus qui ont opté pour être classés en tant que client commercial avec des actifs liquides de plus de 1 million d'USD et qui disposent de connaissances, d'une expérience et d'une compréhension leur permettant de participer à de tels produits et/ou services. Royaume-Uni: Ce document est publié par Credit Suisse (UK) Limited et Credit Suisse Securities (Europe) Limited. Credit Suisse Securities (Europe) Limited et Credit Suisse (UK) Limited sont des sociétés associées réglementées par la Financial Services Authority, mais indépendantes du Credit Suisse sur le plan juridique. Elles sont agréées et soumises à la surveillance de la Financial Services Authority. Les protections offertes par la Financial Services Authority à la clientèle privée ne s'appliquent pas aux placements et services fournis par des personnes situées en dehors du Royaume-Uni. Le Financial Services Compensation Scheme est inapplicable lorsque l'émetteur n'a pas satisfait à ses obligations. Russie: Les recherches figurant dans cette brochure ne constituent en aucune façon une publicité ni une promotion pour des titres spécifiques ni pour des instruments financiers en relation. Cette brochure de recherches ne constitue pas une évaluation au sens de la loi fédérale sur les activités d'évaluation dans la Fédération de Russie et elle est produite en faisant appel aux modèles et à la méthodologie d'évaluation du Credit Suisse. Singapour: Ce rapport a été élaboré et publié à Singapour exclusivement à l'intention des investisseurs institutionnels, des investisseurs accrédités et des investisseurs avertis (tels que définis dans les Financial Advisers Regulations), et est également publié par Credit Suisse AG, succursale de Singapour, à l'intention des investisseurs étrangers (tels que définis dans les Financial Advisers Regulations) En vertu de votre statut d'investisseur institutionnel, d'investisseur accrédité, d'investisseur averti ou d'investisseur étranger, Credit Suisse AG, succursale de Singapour, est dispensé de respecter certaines exigences de conformité selon le Financial Advisers Act, chap. 110 de Singapour («FAA»), les Financial Advisers Regulations et les notifications et les directives qui en découlent, en relation avec les prestations de conseil financier que Credit Suisse AG, succursale de Singapour, est susceptible de vous fournir. Thaïlande: Ce rapport est distribué par Credit Suisse Securities (Thailand) Limited, qui est réglementé par l'Office of the Securities and Exchange Commission, Thaillande, et dont l'adresse enregistrée est 990 Abdulrahim Place Building, 27/F, Rama IV Road, Silom, Bangrak, Bangkok, tél. 0-2614-6000.

ÉTATS-UNIS: LE PRÉSENT DOCUMENT, EN SA FORME ORIGINALE OU COPIÉE, NE SAURAIT ÊTRE ENVOYÉ, INTRODUIT OU DISTRIBUÉ AUX ÉTATS-UNIS OU À DES PERSONNES IMPOSABLES AUX ÉTATS-UNIS.

La distribution est également susceptible d'être limitée dans d'autres pays en raison de la législation ou de la réglementation locale.

Toute reproduction intégrale ou partielle du présent document est soumise à l'autorisation écrite du Credit Suisse. Copyright (C) 2013 Credit Suisse Group AG et/ou sociétés affiliées. Tous droits réservés. 